8-2019

\title{
Application of Elastodynamic Finite Integration Technique (EFIT) to Three-Dimensional Wave Propagation and Scattering in Arbitrary Geometries
}

Sean M. Raley

University of New Haven

Follow this and additional works at: https://digitalcommons.newhaven.edu/masterstheses

Part of the Mechanical Engineering Commons

\section{Recommended Citation}

Raley, Sean M., "Application of Elastodynamic Finite Integration Technique (EFIT) to Three-Dimensional Wave Propagation and Scattering in Arbitrary Geometries" (2019). Master's Theses. 149.

https://digitalcommons.newhaven.edu/masterstheses/149 
THE UNIVERSITY OF NEW HAVEN

\author{
APPLICATION OF ELASTODYNAMIC FINITE INTEGRATION \\ TECHNIQUE (EFIT) TO THREE-DIMENSIONAL WAVE \\ PROPAGATION AND SCATTERING \\ IN ARBITRARY GEOMETRIES
}

\begin{abstract}
A THESIS
submitted in partial fulfillment

of the requirements for the degree of

MASTER OF SCIENCE IN MECHANICAL ENGINEERING
\end{abstract}

\author{
BY \\ Sean M. Raley \\ University of New Haven \\ West Haven, Connecticut \\ August, 2019
}




\section{ACKNOWLEDGMENTS}

Sincerest thanks to Professor Eric A. Dieckman, Ph.D. for his patience and support in teaching me the many, many things I needed to know to complete this thesis.

Special thanks also to Meredith C. Powell, Ph.D. and John E. Leonard; and appreciation to UNH. 


\begin{abstract}
Over several decades, railroad Ultrasonic Testing (UT) industry techniques have primarily been developed through simple analytical modelling and experimental approaches. However, with present-day computational capabilities, we can use numerical techniques like the Elastodynamic Finite Integration Technique (EFIT) to fine-tune systems for complex applications before the fabrication process begins. EFIT is well-established as a useful method in numerical analysis of ultrasonic wave propagation with distinct advantages over the Finite Difference Time Domain method. Several software packages exist that use EFIT as the primary method for simulating the behavior of ultrasonic waves over time in 2 or 3 dimensions, but none of them are well-suited for railroad UT research and development. This thesis explores the complete development of a custom tool for this purpose which was designed to: (1) allow for the input of various profile geometries, boundary conditions, and material inclusion geometries (such as a bolt hole in a railroad track); and (2) allow for the input of specific ultrasonic impulses from varying emitter designs. The custom software produced results that closely matched expected wave propagation behavior. The results were processed into useful visual representations of that behavior.
\end{abstract}




\section{TABLE OF CONTENTS}

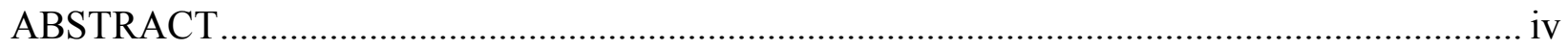

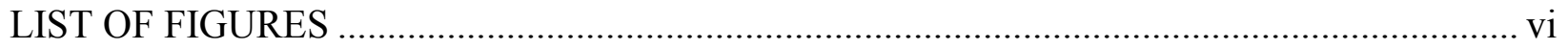

CHAPTER I: Ultrasonic Testing in Rail Applications and Relevant Numerical Solutions ........... 1

CHAPTER II: AFIT Implementation and Results .............................................................. 11

CHAPTER III: EFIT Implementation and Results .......................................................... 16

CHAPTER IV: Conclusions and Future Work ............................................................... 30

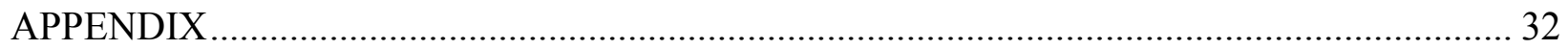

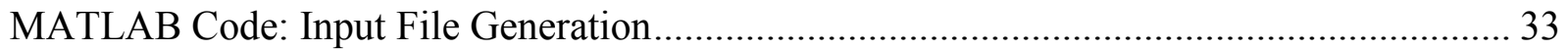

MATLAB Code: Arbitrary Geometry Importing and Processing ...................................... 37

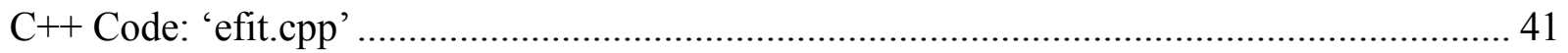

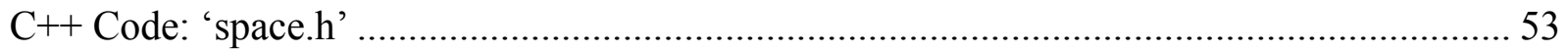

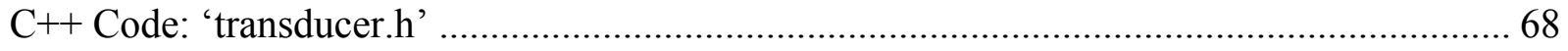

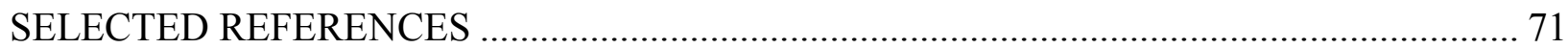




\section{LIST OF FIGURES}

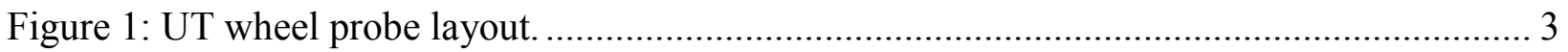

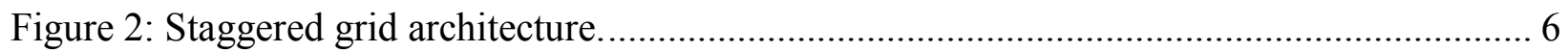

Figure 3: This flowchart outlines the general architecture for this FIT implementation............... 11

Figure 4: Example of PML's implemented in 2-D space........................................................... 13

Figure 5: Half-space 3-D view of the rigid sphere AFIT simulation........................................... 14

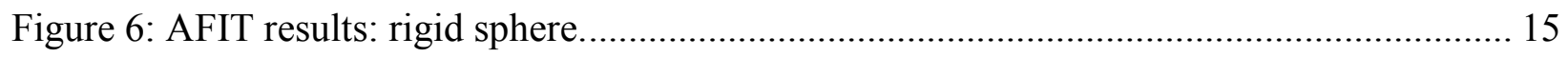

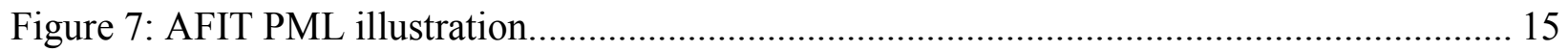

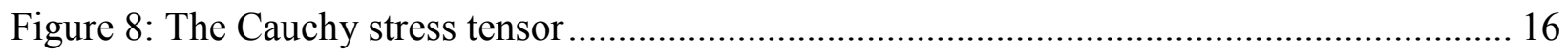

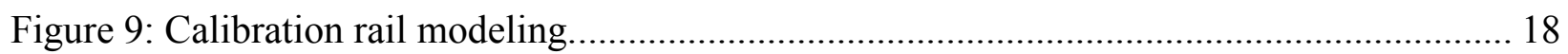

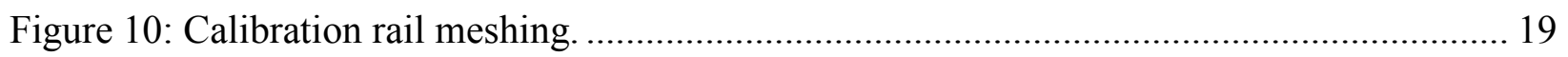

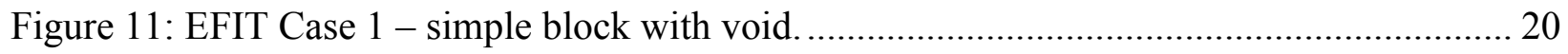

Figure 12: EFIT Case 2 - simple block comparison (no void) .................................................. 21

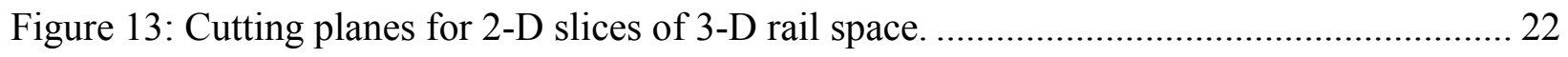

Figure 14: EFIT Case 3 - beam in rail, $0^{\circ}$, 2-D slices. …………………………………........ 23

Figure 15: EFIT Case 3 - beam in rail, $0^{\circ}$, 2-D slice (longitudinal) …………………................ 24

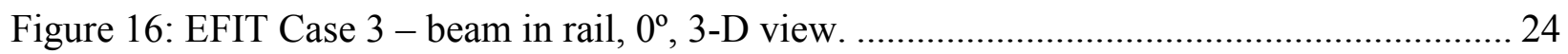

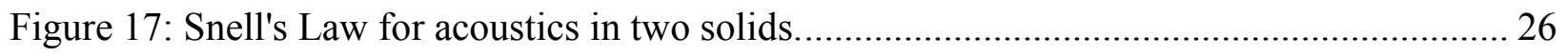

Figure 18: EFIT Case 4 - beam in rail, 56.4 $4^{\circ}$, 3-D view. ............................................................ 26

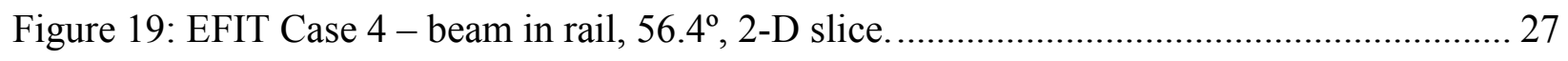

Figure 20: EFIT Case 5 - beam in rail, 37.5 , 2-D slice (longitudinal) ........................................ 28

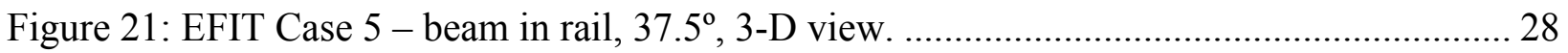


Figure 22: EFIT Case 6 - beam in rail, 70 ${ }^{\circ}$ 2-D slice (longitudinal)................................... 29

Figure 23: EFIT Case 6 - beam in rail, 70º, $3-\mathrm{D}$ view. ........................................................ 29 
CHAPTER I: Ultrasonic Testing in Rail Applications and Relevant Numerical

\section{Solutions}

How do we predict an imminent train derailment on railroad track that has been in service for 80 years or more? How do we determine that a brand new gas cylinder is likely to explode if it is put into service? In cases like these and many others, the answer is Ultrasonic Testing (UT), a subset of nondestructive evaluation (NDE). UT is, at its core, concerned with inducing ultrasonic waves into a material and making inferences about the material's internal structure based on the exiting ultrasonic waves. Feasible candidates for UT include product forms that are wrought, cast, welded, composite, or other materials as long as they have: (a) surface and internal geometries suitable for the application of UT; and (b) relevant defect definitions that are of shapes and orientations that conducive to ultrasonic inspection [1]. UT is used to ensure the safety of many types of system components and structures, including train wheels, pressurized tanks, railroad tracks, tubes, pipes, ammunition, airplane parts, and structural beams. Many of these components are inspected using UT techniques both at the time of manufacture and while in service.

UT is a well-established application of acoustic science. The basic principle is the same as SONAR: a sound wave is emitted, it reflects off of some feature of interest, and the reflection is detected and interpreted. The departure from large scale sensing techniques is driven by the frequencies used. SONAR mainly uses frequencies in the human audible range $(20-20,000 \mathrm{~Hz})$, while ultrasonic waves are by definition above $20 \mathrm{kHz}$. Attenuation is proportional to the square of frequency, so detection range decreases at higher frequencies, but resolution and sensitivity increase. An important note is that at ultrasonic frequencies, sound cannot propagate any useful distance in air. UT is typically conducted in the $\mathrm{MHz}$ range $(0.5-25 \mathrm{MHz})[1]$. 
Ultrasonic waves are typically generated by piezoelectric transducers, which exploit the piezoelectric effect, in which structural deformation is induced by applied voltage and vice versa. This characteristic allows them to both emit ultrasonic waves and convert incoming ultrasonic signals into electrical signals, which can then be recorded and interpreted by the rest of the system. Transducer configurations include pulse-echo (the emitting transducer is also the detecting transducer), pitch-catch (a second transducer acts as the detector for reflections of an angled ultrasonic beam), and through-transmission (similar to pitch-catch, but with the transducers located on opposite sides of the target material).

Since railroad track inspection is one of the primary motivators of this project, a more thorough background of that specific UT application is appropriate. While the details of the application are complex, the general concept is relatively simple: a fluid-filled wheel probe with several transducers held at fixed angles inside it rolls down the track, continuously collecting measurements (Figure 1). The ultrasonic beam propagates from each transducer, through the wheel fluid, through the membranous tire, through a film of liquid couplant sprayed on the rail to eliminate air gaps, and into the railroad track. From there, if the beam strikes a discontinuity roughly orthogonal to its propagation direction, it will reflect back along the same path up into the wheel probe and be received by the same transducer that emitted the pulse. There are a number of different defect types which occur at various positions and angles within railroad tracks, so an array of transducers at various positions and angles must be used to find them. 


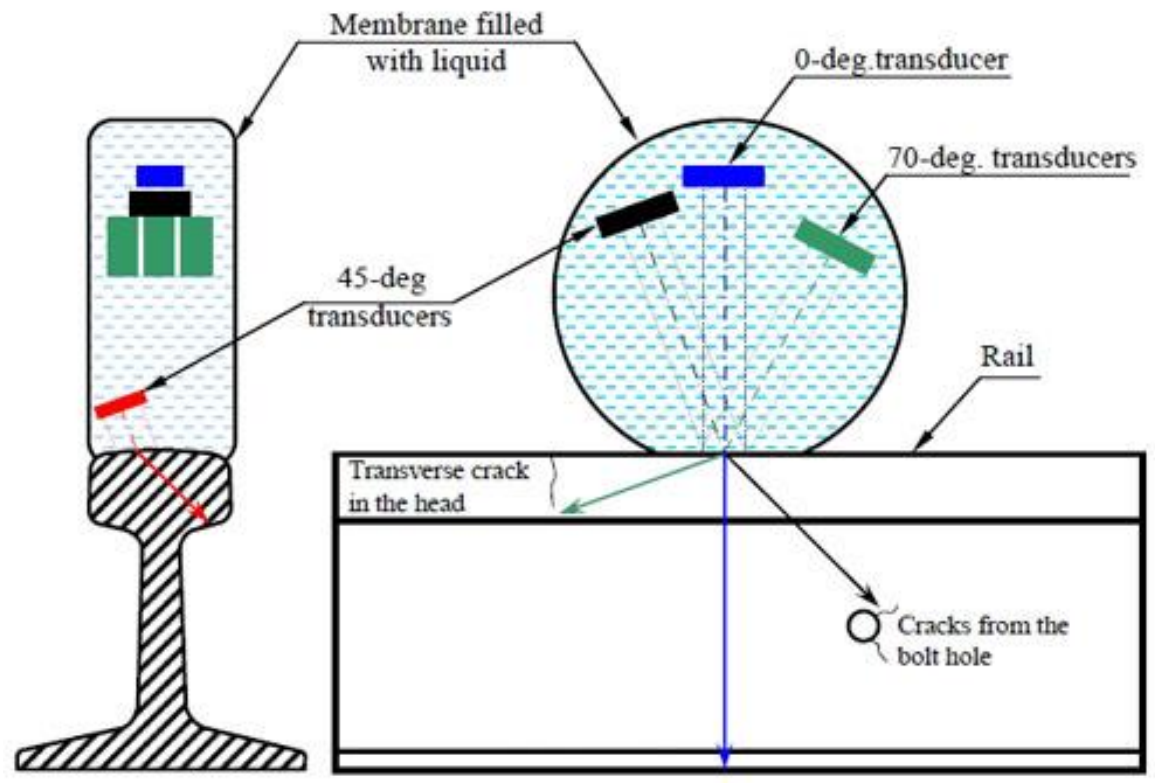

Figure 1: UT wheel probe layout. Wheel probes are rolled along in-service railroad tracks to detect many different types of flaws. The wheel probe shown here contains a typical array of transducers. Adapted from [2].

Railroad tracks experience a multitude of cyclic and transient loading conditions, including vertical loading from trains, lateral loading from trains, lateral creep forces at the rail/wheel interface, thermal stresses, and residual stresses from manufacturing or welding processes [3]. Due to these complex loading patterns which span decades or longer, there are many different types of defects which may develop in the rail and eventually cause failure. One of the most common defect types is the transverse fissure, which is a crosswise fracture originating from a nucleus inside the head and spreading outward orthogonally to the length of the rail. They are impossible to detect visually until the rail has already broken, but they can be found with a $70^{\circ}$ angled transducer in a wheel probe. Another common defect type is the vertical split head, a progressive longitudinal fracture near the middle of the head along the length of the rail. They are typically not visible from the surface until they have grown several feet long. Vertical split heads are particularly prone to causing derailments after complete mechanical failure. Luckily, they can be found using $0^{\circ}$ or $45^{\circ}$ angled transducers. 
Many discontinuities appropriate for UT in general fall under the categories of cracks, gaps, or other inclusions that behave approximately like rigid reflectors. Scattering of acoustic waves has well-established solutions for very simple geometries - for example, scattering of a propagating plane wave from a rigid sphere has been thoroughly explored [4]-[6]. However, as complexity is added to the reflector geometry, the incident waveform, or the boundary conditions, analytical solutions quickly become very cumbersome or impossible. At this point, more advanced numerical tools and techniques are required. As applicable to UT, these include semi-analytical techniques like the geometric theory of diffraction and the boundary element method (BEM), and numerical techniques operating directly on the fundamental equations of motion, among them finite difference time domain methods (FDTD), and finite element methods (FEM) [7].

This paper will focus on the Finite Integration Technique (FIT), an explicit hyperbolic time-domain solver, as applied to the acoustic and elastodynamic cases. In the case of the Acoustic Finite Integration Technique (AFIT), the model directly develops the fundamental governing equations into a parallelized solver for simulation of acoustic wave propagation in fluids in 3 dimensions. The Elastodynamic Finite Integration Technique (EFIT) is an analogous model for ultrasonic wave propagation in isotropic homogeneous solid media.

To better understand the general FIT, it is useful to first compare it to FDTD. Both methods generally use a cubic hexahedral or cylindrical grid, though advanced meshing techniques such as subgridding can be applied [8]. They both use Yee's staggered grid structure (primary grid and offset secondary grid), first presented in 1966 for electromagnetic simulation (Figure 2) [9]. FIT and FDTD both use a marching-in-time leapfrog scheme, meaning that the pressure updates alternate with the velocity updates a half timestep apart. By using this explicit 
time integration scheme, no iteration is involved in the solution procedure. This also means that the maximum possible time step is determined by Courant-Friedrich-Lewy-criterion,

$$
\begin{gathered}
\Delta t \leq \Delta t_{\max }=\frac{1}{\sqrt{n}} \frac{\Delta x}{c_{\max }} \\
\Delta x \approx \frac{\lambda_{\min }}{10}
\end{gathered}
$$

where $n$ is the number of spatial dimensions and $c_{\max }$ is the highest wave propagation speed in the simulation. The minimum wavelength $\lambda_{\min }$ drives the maximum spatial discretization size $\Delta x$. Due to the inverse relationship between frequency and wavelength, high-frequency simulations are forced to finer resolutions, increasing computational requirements. The maximum temporal discretization size $\Delta t_{\max }$ is directly proportional to the chosen $\Delta x$, so higher frequencies also force a finer time resolution.

In both models, the use of a fixed mesh causes spatial discretization error. A material property discretization error also occurs [10]. Additionally, FDTD and FIT are full-wave propagation methods as opposed to spectral methods, which are numerical techniques that operate in the frequency domain.

The key difference between FIT and FDTD is form of the governing field equations on which they are based. The FDTD approach uses the differential form of these equations, while the FIT is based on the integral forms of the field equations. This means that for a fixed mesh (which includes an inherent spatial discretization error), no additional equation discretization error is introduced when passing from the continuous to the discrete form [11]. In other words, FIT contains less inherent error than FDTD. 

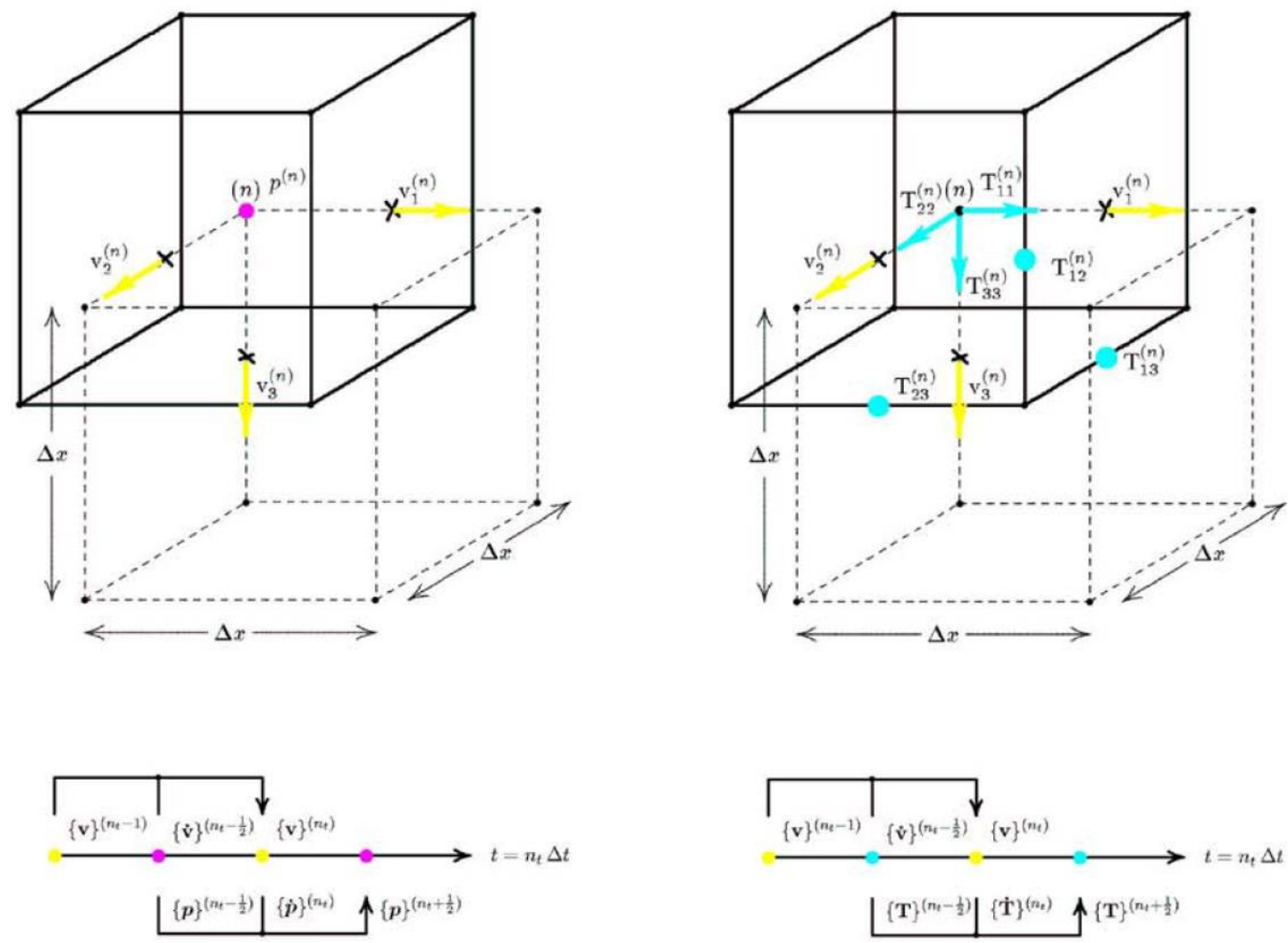

$$
p_{1}^{(n)}, \mathrm{v}_{2}^{(n)}, \mathrm{v}_{3}^{(n)}
$$

$$
\begin{aligned}
& \mathrm{v}_{1}^{(n)}, \mathrm{v}_{2}^{(n)}, \mathrm{v}_{3}^{(n)} \\
& \mathrm{T}_{11}^{(n)}, \mathrm{T}_{22}^{(n)}, \mathrm{T}_{33}^{(n)} \\
& \mathrm{T}_{13}^{(n)}=\mathrm{T}_{31}^{(n)}, \mathrm{T}_{12}^{(n)}=\mathrm{T}_{21}^{(n)}, \mathrm{T}_{23}^{(n)}=\mathrm{T}_{32}^{(n)}
\end{aligned}
$$

Figure 2: Staggered grid architecture. Yee's staggered grid method can be applied to FIT for the simplified model of acoustic wave propagation in fluids (left) or the full elastodynamic model in solids (right). The corresponding leapfrog update schemes are shown as well (below). The Cartesian velocity vector $\mathrm{v}$ is positioned at the center of each face of the primary grid and the center of each edge of the secondary grid. The scalar pressure value for fluids $\mathrm{p}$ is shown in the center of the primary grid and at the corner of the secondary grid. The normal components of the Cauchy stress tensor $\mathrm{T}$ are positioned at the center of the primary grid, while the shear components are on the center of each edge of the primary grid. Adapted from [12].

FIT and FDTD can be compared with FEM as a single grouping. As discussed in [13], FIT and FDTD are both more widely implemented than FEM mainly due to the relative simplicity of their programming. FEM is formulated using unstructured grids, which give it greater versatility for complex geometries but require advanced knowledge of mesh generation in order to properly implement. FEM requires far more computational resources than FDTD and 
FIT for relatively simple geometric cases; however, the typical strategy for handling complex geometry in FDTD and FIT is to use a finer mesh, either globally or locally. Due to this limitation, FEM can be the more computationally efficient method for certain complex geometries. FEM is also generally superior to FDTD when material interfaces are involved, but FIT can easily account for continuity conditions at these interfaces.

For Acoustic FIT, propagation is only simulated through fluids, so no shear stresses can be carried in the material. The governing field equations are derived from conservation of mass, conservation of momentum, and a thermodynamic equation of state [14]. This leads to equations for the pressure $p$ and velocity vector $\underline{\mathbf{v}}$ in real cartesian space $\underline{\mathbf{R}}$ and time $t$,

$$
\begin{gathered}
\frac{\delta}{\delta t} \underline{\mathbf{j}}(\underline{\mathbf{R}}, t)=-\nabla p(\underline{\mathbf{R}}, t)+\underline{\mathbf{f}}(\underline{\mathbf{R}}, t) \\
\frac{\delta}{\delta t} S(\underline{\mathbf{R}}, t)=\nabla \cdot \underline{\mathbf{v}}(\underline{\mathbf{R}}, t)+h(\underline{\mathbf{R}}, t)
\end{gathered}
$$

where $\underline{\mathbf{j}}$ is the momentum density vector, $\underline{\mathbf{f}}$ is the volume force density vector, $S$ is the scalar deformation, and $h$ is the injected deformation rate. This is the differential form used in FDTD.

To create the integral form of these equations for AFIT, Equations 3 and 4 are first combined with the constitutive material equations,

$$
\begin{aligned}
& \underline{\mathbf{j}}(\underline{\mathbf{R}}, t)=\rho_{a 0}(\underline{\mathbf{R}}) \underline{\mathbf{v}}(\underline{\mathbf{R}}, t) \\
& S(\underline{\mathbf{R}}, t)=-\kappa(\underline{\mathbf{R}}) p(\underline{\mathbf{R}}, t)
\end{aligned}
$$

where $\rho_{a 0}$ is the acoustic mass density at rest and $\kappa$ is the compressibility. The constitutive equations limit the scope of this model to linear, inhomogeneous, anisotropic, instantaneously and locally reacting media. Gauss' and Stokes' Theorems are then applied [12], [15] to arrive at the integral forms of the governing field equations, 


$$
\begin{gathered}
\iiint_{V} \rho_{a 0}(\underline{\mathbf{R}}) \underline{\dot{\mathbf{v}}}(\underline{\mathbf{R}}, t) d V=-\oiint_{S=\delta V} p(\underline{\mathbf{R}}, t) \underline{\mathbf{d} \mathbf{S}}+\iiint_{V} \underline{\mathbf{f}}(\underline{\mathbf{R}}, t) d V \\
\underline{\mathbf{v}}(\underline{\mathbf{R}}, t)=\underline{\mathbf{v}}\left(\underline{\mathbf{R}}, t_{0}\right)+\int_{t_{0}}^{t} \underline{\dot{\mathbf{v}}}\left(\underline{\mathbf{R}}, t^{\prime}\right) d t^{\prime} \\
\iiint_{V} \kappa(\underline{\mathbf{R}}) \dot{p}(\underline{\mathbf{R}}, t) d V=-\oiint_{S=\delta V} \underline{\mathbf{v}}(\underline{\mathbf{R}}, t) \cdot \underline{\mathbf{d} \mathbf{S}}-\iiint_{V} h(\underline{\mathbf{R}}, t) d V \\
p(\underline{\mathbf{R}}, t)=p\left(\underline{\mathbf{R}}, t_{0}\right)+\int_{t_{0}}^{t} \dot{p}\left(\underline{\mathbf{R}}, t^{\prime}\right) d t^{\prime} .
\end{gathered}
$$

The integral forms are then directly converted into the numerical matrix update equations for AFIT,

$$
\begin{gathered}
\{\dot{\mathbf{v}}\}^{\left(n_{t}-1 / 2\right)}=\left[\widetilde{\mathbf{\rho}}_{a 0}\right]^{-1}[\widetilde{\mathbf{R}}]^{-1}[\widetilde{\mathbf{g r a d}}]\{\mathbf{p}\}^{\left(n_{t}-1 / 2\right)}+\left[\widetilde{\boldsymbol{\rho}}_{a 0}\right]^{-1}\{\mathbf{f}\}^{\left(n_{t}-1 / 2\right)} \\
\{\mathbf{v}\}^{\left(n_{t}\right)}=\{\mathbf{v}\}^{\left(n_{t}-1\right)}+\Delta t\{\dot{\mathbf{v}}\}^{\left(n_{t}-1 / 2\right)} \\
\{\dot{\mathbf{p}}\}^{\left(n_{t}\right)}=-[\mathbf{k}]^{-1}[\mathbf{d i v}][\mathbf{R}]^{-1}\{\mathbf{v}\}^{\left(n_{t}\right)}-[\mathbf{k}]^{-1}\{\mathbf{h}\}^{\left(n_{t}\right)} \\
\{\mathbf{p}\}^{\left(n_{t}+1 / 2\right)}=\{\mathbf{p}\}^{\left(n_{t}-1 / 2\right)}+\Delta t\{\dot{\mathbf{p}}\}^{\left(n_{t}\right)}
\end{gathered}
$$

where $n_{t}$ is an integer time step counter such that $t=\Delta t n_{t}$ and the $\sim$ operator indicates that a matrix is defined on the secondary grid. This direct, one-to-one mapping of the field equations is the reason FIT does not have the equation discretization error found in FDTD and other numerical techniques.

For EFIT, the full elastodynamic equations must be used. The governing field equations for viscid fluids and solids are the linear vectorial Cauchy-Newton's law of motion and the tensorial law of deformation rate,

$$
\begin{gathered}
\frac{\delta}{\delta t} \underline{\mathbf{j}}(\underline{\mathbf{R}}, t)=\nabla \cdot \underline{\underline{\mathbf{T}}}(\underline{\mathbf{R}}, t)+\underline{\mathbf{f}}(\underline{\mathbf{R}}, t) \\
\frac{\delta}{\delta t} \underline{\mathbf{S}}(\underline{\mathbf{R}}, t)=\operatorname{sym}\{\nabla \cdot \underline{\mathbf{v}}(\underline{\mathbf{R}}, t)\}+\underline{\mathbf{h}}(\underline{\mathbf{R}}, t)
\end{gathered}
$$


where $\underline{\underline{\mathbf{T}}}$ is Cauchy's stress tensor of $2^{\text {nd }}$ rank, $\underline{\underline{\mathbf{S}}}$ is the deformation tensor of $2^{\text {nd }}$ rank, $\underline{\underline{\mathbf{h}}}$ is the injected deformation rate tensor of $2^{\text {nd }}$ rank, and "sym" is a symmetric gradient operator. This is the differential form used in FDTD.

To create the integral form of these equations for EFIT, Equations 17 and 18 are first combined with the constitutive material equations,

$$
\begin{aligned}
& \underline{\mathbf{j}}(\underline{\mathbf{R}}, t)=\rho_{e 0}(\underline{\mathbf{R}}) \underline{\mathbf{v}}(\underline{\mathbf{R}}, t) \\
& \underline{\mathbf{S}}(\underline{\mathbf{R}}, t)=\underline{\underline{\mathbf{s}}}(\underline{\mathbf{R}}): \underline{\mathbf{T}}(\underline{\mathbf{R}}, t)
\end{aligned}
$$

where $\rho_{e 0}$ is the elastodynamic mass density at rest, $\underline{\mathbf{s}}$ is the compliance tensor of $4^{\text {th }}$ rank, and the colon ":" is an operator denoting a double-scalar product. The constitutive equations limit the scope of this model to linear, inhomogeneous, anisotropic, instantaneously and locally reacting media. Gauss' and Stokes' Theorems are then applied [12], [15] to arrive at the integral forms of the governing field equations,

$$
\begin{gathered}
\iiint_{V} \rho_{e 0}(\underline{\mathbf{R}}) \underline{\dot{\mathbf{v}}}(\underline{\mathbf{R}}, t) d V=\oiint_{S=\delta V} \underline{\mathbf{T}}(\underline{\mathbf{R}}, t) \cdot \underline{\mathbf{d}} \mathbf{S}+\iiint_{V} \underline{\mathbf{f}}(\underline{\mathbf{R}}, t) d V \\
\underline{\mathbf{v}}(\underline{\mathbf{R}}, t)=\underline{\mathbf{v}}\left(\underline{\mathbf{R}}, t_{0}\right)+\int_{t_{0}}^{t} \underline{\dot{\mathbf{v}}}\left(\underline{\mathbf{R}}, t^{\prime}\right) d t^{\prime} \\
\iiint_{V} \underline{\underline{\mathbf{s}}}(\underline{\mathbf{R}}): \underline{\underline{\mathbf{T}}}(\underline{\mathbf{R}}, t) d V=\oiint_{S=\delta V} \operatorname{sym}\{\underline{\mathbf{n}} \underline{\mathbf{v}}(\underline{\mathbf{R}}, t)\} d S+\iiint_{V} \underline{\underline{\mathbf{h}}}(\underline{\mathbf{R}}, t) d V \\
\underline{\mathbf{T}}(\underline{\mathbf{R}}, t)=\underline{\mathbf{T}}\left(\underline{\mathbf{R}}, t_{0}\right)+\int_{t_{0}}^{t} \underline{\underline{\mathbf{T}}}\left(\underline{\mathbf{R}}, t^{\prime}\right) d t^{\prime}
\end{gathered}
$$

where $\underline{\mathbf{n}}$ is the unit normal at an interface.

The integral forms are then directly converted into the numerical matrix update equations for EFIT, 


$$
\begin{gathered}
\{\dot{\mathbf{v}}\}^{\left(n_{t}-1 / 2\right)}=\left[\widetilde{\boldsymbol{\rho}}_{e 0}\right]^{-1}[\widetilde{\mathbf{D I V}}]\left[\widetilde{\mathbf{R}}_{\dot{\boldsymbol{V}}}^{\boldsymbol{T}}\right]^{-1}\left[\mathbf{A}_{\dot{\mathbf{V}}}^{\boldsymbol{T}}\right]\{\mathbf{T}\}^{\left(n_{t}-1 / 2\right)}+\left[\widetilde{\boldsymbol{\rho}}_{e 0}\right]^{-1}\{\mathbf{f}\}^{\left(n_{t}-1 / 2\right)} \\
\{\mathbf{v}\}^{\left(n_{t}\right)}=\{\mathbf{v}\}^{\left(n_{t}-1\right)}+\Delta t\{\dot{\mathbf{v}}\}^{\left(n_{t}-1 / 2\right)} \\
\{\dot{\mathbf{T}}\}^{\left(n_{t}\right)}=[\mathbf{c}]\left[\mathbf{R}_{\dot{\mathbf{T}}}^{V}\right]^{-1}[\mathbf{G R A D}]\left[\mathbf{A}_{\dot{\mathbf{T}}}^{\boldsymbol{V}}\right]\{\mathbf{v}\}^{\left(n_{t}\right)}+\{\mathbf{g}\}^{\left(n_{t}\right)} \\
\{\mathbf{T}\}^{\left(n_{t}+1 / 2\right)}=\{\mathbf{T}\}^{\left(n_{t}-1 / 2\right)}+\Delta t\{\dot{\mathbf{T}}\}^{\left(n_{t}\right)}
\end{gathered}
$$

where $\left[\mathbf{A}_{\dot{V}}^{\boldsymbol{T}}\right]$ and $\left[\mathbf{A}_{\dot{\boldsymbol{T}}}^{\boldsymbol{V}}\right]$ are averaging matrices and $\mathbf{g}$ is the interface source density of injected deformation rate.

Now that the update equations have been developed for AFIT and EFIT, the coding and implementation can begin. We apply the numerical technique to a number of simple test cases, then expand the capabilities beyond well-established basic FIT implementations to accommodate arbitrary geometries - in this case, the simulation of ultrasound in railroad track. 
CHAPTER II: AFIT Implementation and Results

In this chapter, the programming and technical implementations will be discussed for AFIT only, but much of the information here will form a basis for the EFIT discussion in the next chapter. The general simulation architecture is to 1) write input files using MATLAB, 2) perform the marching-in-time algorithm using a parallelized $\mathrm{C}++$ executable program written specifically for this project, and 3) perform postprocessing and create graphics using VisIt, an open-source visualization tool developed by the U.S. Department of Energy [16]. Each of the three main simulation steps can be performed sequentially on a computing cluster. The system used for these simulation runs had a 16-core $2.10 \mathrm{GHz}$ CPU and $500 \mathrm{~GB}$ of RAM.

\section{Create input files in MATLAB}

- Space/time parameters

- Material properties

- Scatterers

\section{Parallelized $\mathrm{C}++$ program (OpenMPI)}

- Reads input files and distributes them to any number of processors

- Sets up arrays, then does the math

- PMLs to minimize space requirements

- Periodically extracts output values

\section{Postprocessing in VisIt (visualization and graphical analysis tool)}

\section{Figure 3: This flowchart outlines the general architecture for this FIT implementation.}

The MATLAB script begins by defining various material, spatial, and temporal properties. For AFIT, these include material density, sound speed, maximum input frequency, number of time steps, physical size of the simulation space, and the sizes and locations of any scatterers. Several other parameters are then calculated; for example, the maximum input wavelength, spatial discretization size, and temporal discretization size are all interrelated and 
dependent on the sound speed and maximum frequency. A "drive function" is defined based on either an analytical equation (e.g. a sine wave) or an input data file. The drive function is simply an array of pressure values for each time step which will later define a plane wave input at one end of the simulation space. Finally, a text file is written to pass all the necessary parameters (including the full drive function) to the $\mathrm{C}++$ executable.

The executable simulation program uses Open MPI to pass information between processors "nodes" [17]. One node is defined as the master, which is responsible for reading and preprocessing the simulation parameters from the input file, distributing the parameters to all the other nodes, and recording output data periodically. The rest of the nodes are defined as slaves or workers, which each receive an evenly divided slice of the simulation space from the master node and are responsible for performing the actual calculations for the marching-in-time algorithm. All nodes are synced to the current time step as the simulation proceeds. The parallelization slices are orthogonal to the propagation direction of the plane wave.

All the calculations and array bookkeeping are defined by a $\sim 500$-line header file that is instantiated separately by each worker node. For each time step, every worker performs the same procedure independently. First, the drive function is applied as an input plane wave on only a single worker node. Then the pressures are updated for the entire space. Each worker node has one layer of overlap with its neighboring node(s), so these values must be passed and shared using MPI. Next, the velocities are updated for the entire space, with similar value sharing between nodes. This alternating update pattern is the leapfrog scheme in practice. At periodic intervals, the output data is extracted and sent to the master node for recording. Once all of a worker's values are updated for a given time step, absorbing boundary conditions are applied to the outer faces of the overall simulation space. The boundary condition used is a Perfectly 
Matched Layer (PML), which is a technique that applies an incrementally increasing reduction factor to each element of the layer moving towards the outer faces of the simulation space [18]. These layers are part of the computational simulation space but are considered sacrificial and cannot produce valid physical results (Figure 4).
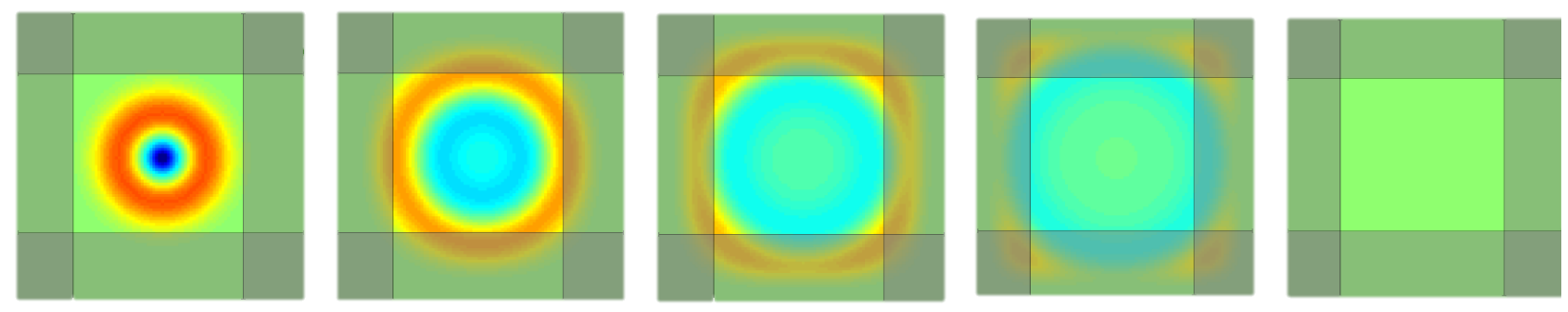

Figure 4: Example of PML's implemented in 2-D space. The method avoids reflections at the inner edge of a PML by matching the impedance of the PML to the impedance of the problem space. Adapted from [19].

Once the simulation is complete, a series of binary files containing the output data has been produced. To help VisIt interpret these binary files, a simple bash script is used to write a series of "brick of values" (.bov) files, which define data type and size, byte order, and other parameters for the corresponding data file. From there, it is relatively simple to create a 3-D plot of the data (Figure 5), which can be manipulated in a wide variety of ways, including video generation.

The primary test case for the AFIT implementation was a rigid sphere in a fluid, which is a well-studied acoustics problem. The input was a standing $2.5 \mathrm{kHz}$ plane wave. The pressure output was recorded for only the even element positions in each principal direction, resulting in an $87.5 \%$ reduction of overall data file size. The expected interference patterns due to reflections from the sphere can be seen in Figure 6. The expected diffraction patterns behind the sphere were also observed. The simulation used PMLs on 5 faces of the computational space (Figure 7). In this implementation, the source face does not have a PML, so the sphere was spaced far 
enough from the source face that reflections from it were not a concern for the simulation time duration.

There are far more interesting problems which can be solved using this implementation of AFIT, but for this thesis, AFIT is primarily used as a stepping stone on the way to the motivating problem of EFIT in arbitrary geometry.

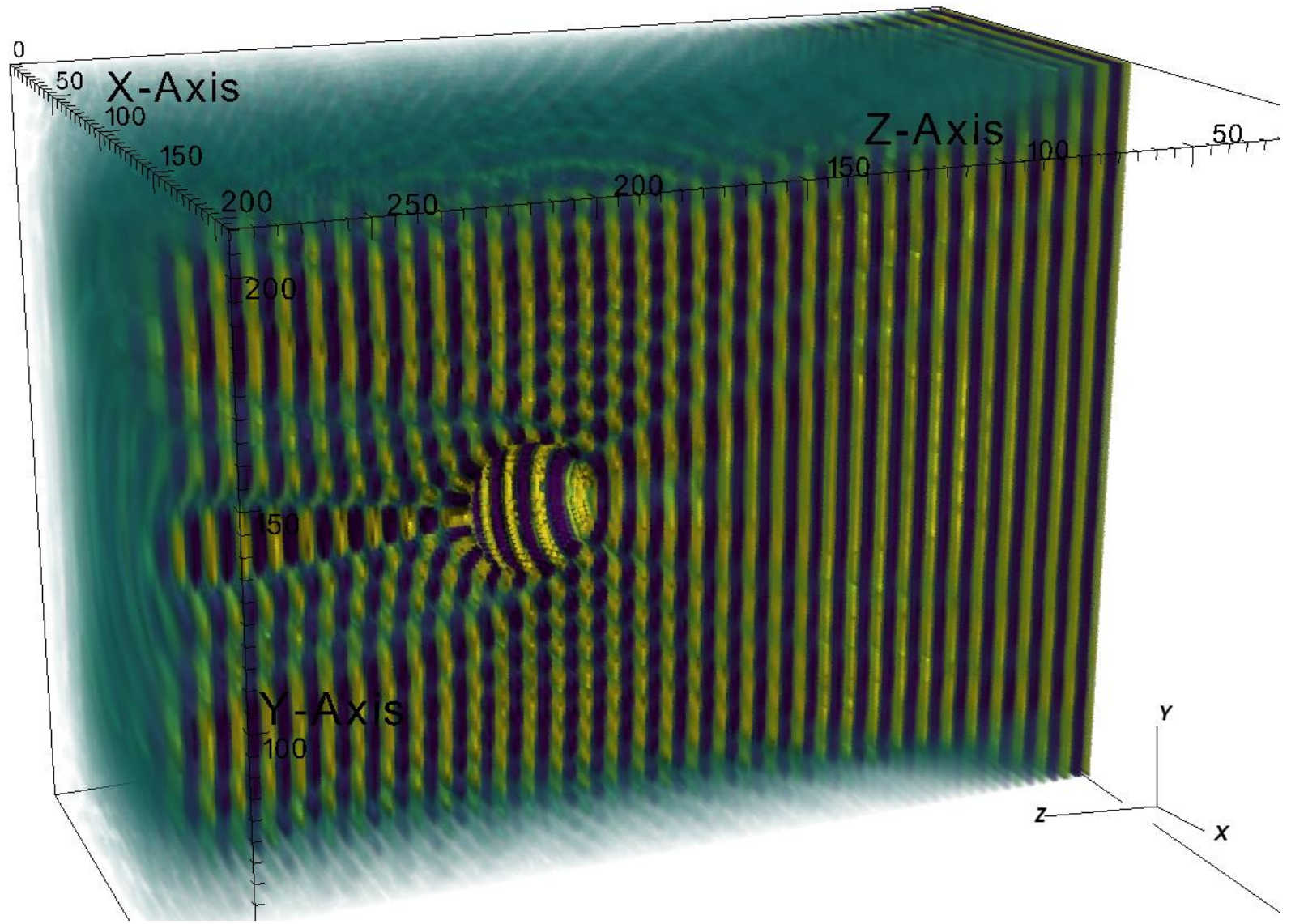

Figure 5: Half-space 3-D view of the rigid sphere AFIT simulation. Note the clearly visible attenuation at the outer faces caused by the PML's. 

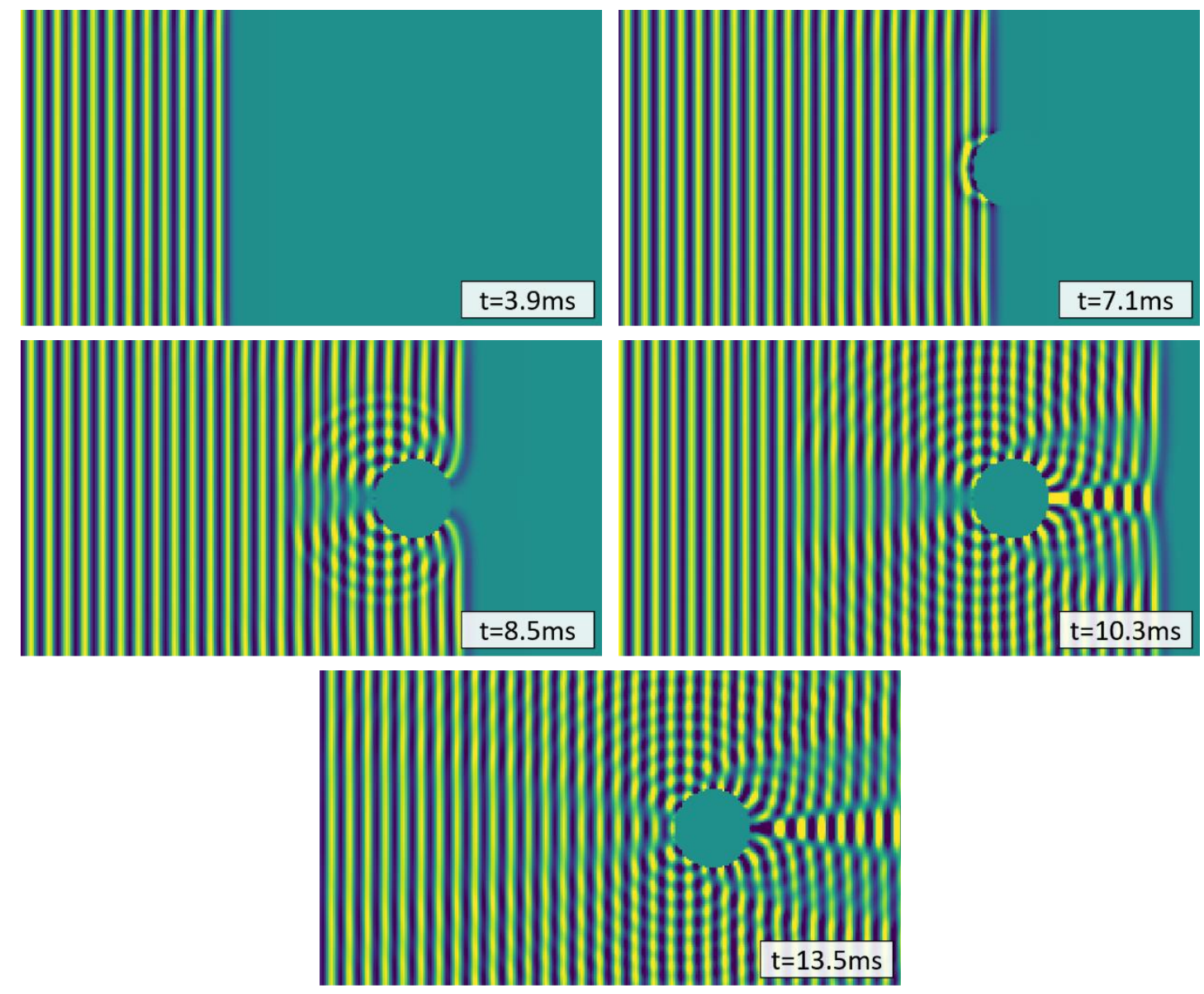

Figure 6: AFIT results: rigid sphere. 2-D slice of a 3-D simulation of a $2.5 \mathrm{kHz}$ plane wave reflecting from a rigid sphere. Results are shown at simulation time steps 345, 620, 740, 900, and 1180, with 1 time step $=11.43 \mu \mathrm{s}$.

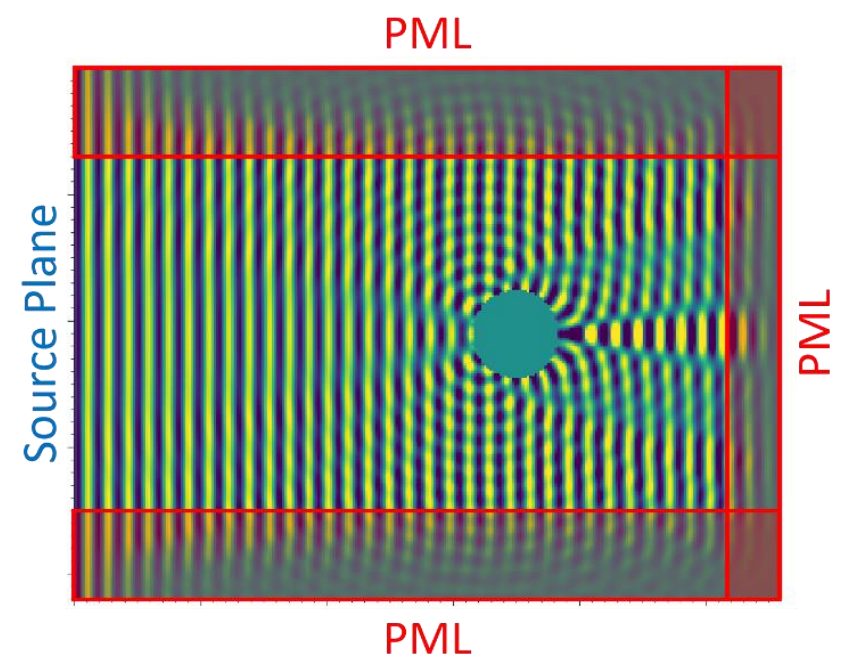

Figure 7: AFIT PML illustration. The PML's in this simulation space (boxed in red) are not valid physical results, so they are generally omitted from final figures. Only 3 PMLs are shown here because the figure is a 2-D slice of a 3-D simulation. Result is shown at simulation time $t=13.5 \mathrm{~ms}$. 


\section{CHAPTER III: EFIT Implementation and Results}

When transitioning from AFIT to EFIT, the general simulation structure remains more or less the same. There are two key differences that add a nontrivial complexity to the implementation of EFIT. First, instead of pressure, all six components of the Cauchy stress tensor must be used since solid materials can support shear stresses (Figure 8). This complicates bookkeeping and calculations. The second difference is specific to the intended application of this work: UT does not typically involve full-space plane waves, so a localized transducer definition must be developed to include the drive function in a localized "drive region."

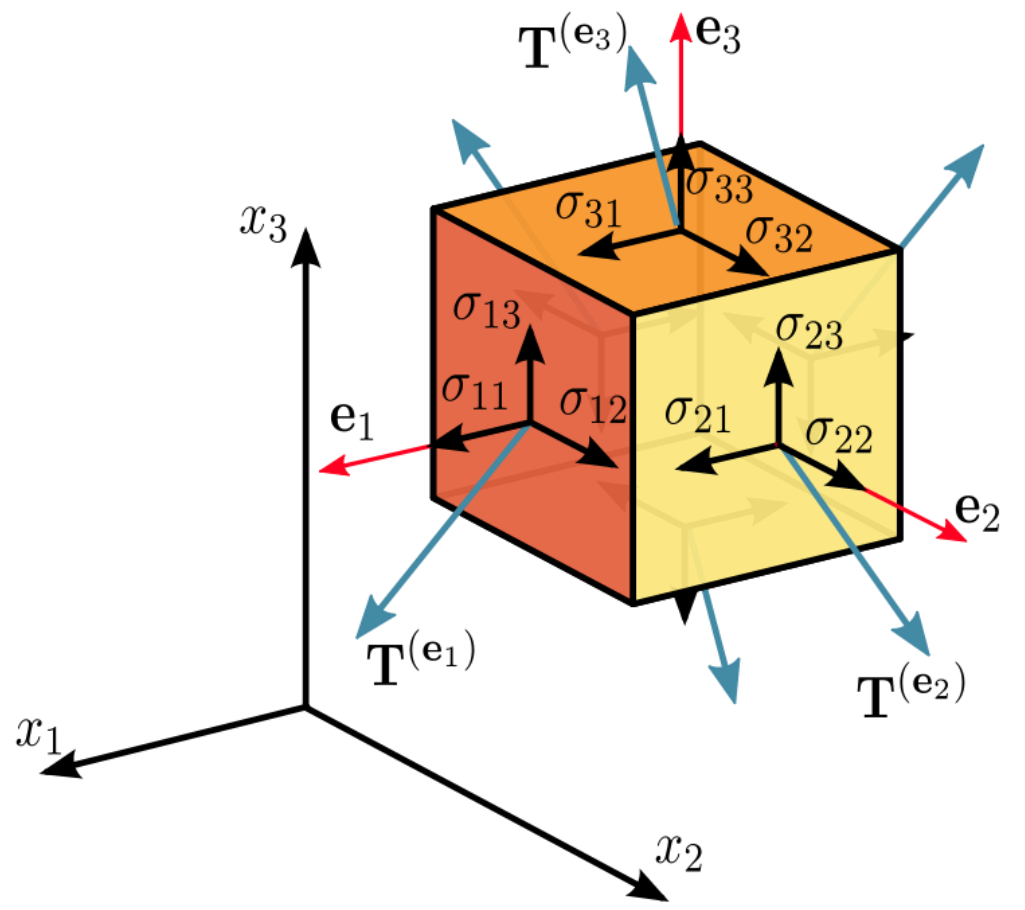

Figure 8: The Cauchy stress tensor is composed of six unique stress vectors. Image from [20].

In order to simplify the implementation of a discretized transducer definition, several assumptions are made. One of the largest simplifications is the limitation of transducer geometry to circular drive regions. There are many situations in which this leads to a slight departure from real-world physics, such as an ultrasonic beam striking the inspected material at an angle (which would create an oblong drive region) or a rectangular transducer being used (which creates a 
drive region that is not quite circular, but not quite round). However, for most situations, a circular drive region can be assumed to introduce an acceptably low error into the simulation. Another simplification is the limitation of velocity input values to the direction orthogonal to the drive region, which can only be placed within the top plane of the simulation space. This is accurate for transducer beams propagating in the same direction ( $0^{\circ}$ transducers in rail testing), but again, it introduces some error for angle beams, which realistically strike the test material with a total velocity parallel to the beam path.

Angled transducer orientations are crucial in many UT applications (with $37.5^{\circ}$ and $45^{\circ}$ being two of the most commonly used beam angles), so the addition of this feature was highly desirable. Angled propagation paths in the inspected material were generated using 2-D array beam steering [21].The drive function time delay for each spatial element is calculated from Equation 27 using the center of the transducer as a reference point, then offset so that all elements have a nonnegative time delay value. This delay time $\tau_{i j}$ is defined as

$$
\tau_{i j}=\frac{z_{i}}{c} \sin \theta \cos \phi+\frac{y_{j}}{c} \sin \theta \sin \phi
$$

where $\theta$ is the angle from incident normal (the $0^{\circ}$ direction) towards positive $z, \phi$ is the clockwise angle from the positive $z$ direction, and $c$ is the longitudinal speed of sound. Once this delay array is set, the same drive function is used throughout the drive region with some elements simply calling a drive function value from an earlier timestep.

Another feature included in this EFIT implementation is the ability to import arbitrary geometry as the simulation space. UT is rarely performed on rectangular prisms, so defining other geometries is essential to creating a useful simulation tool. This is accomplished using stereolithography (STL) files as inputs. STL is a common filetype that represents 3-D geometry using interconnected triangular faces (Figure 9). In the initial MATLAB setup script, the STL 
file is processed into a logical cubic mesh defining whether material exists at any given Cartesian coordinate within the simulation space (Figure 10). The coarseness of this mesh will determine the order of the spatial discretization error. A finer mesh is always more accurate, but computational requirements increase rapidly as discretization length decreases. Anywhere material does not exist will be treated as vacuum in the simulation, so the stresses and velocities in those elements will always be zero. This assumption is minor due to the extreme impedance differential between air and most solids relevant to UT.

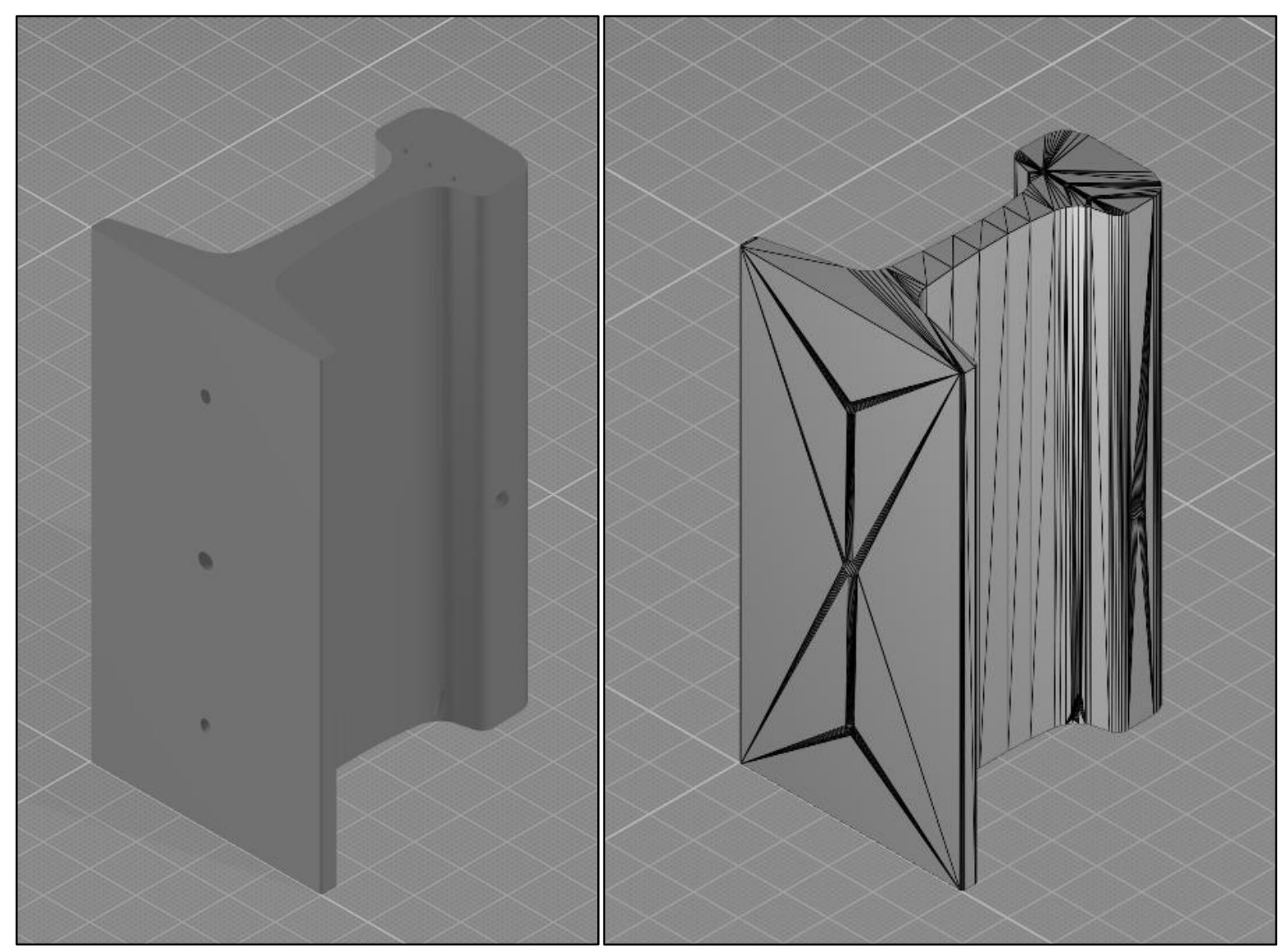

Figure 9: Calibration rail modeling. A 3-D CAD model (left) of a calibration rail used in the UT industry. In the STL version of the same part (right), a high density of triangular faces can be seen intersecting at the hole locations. 


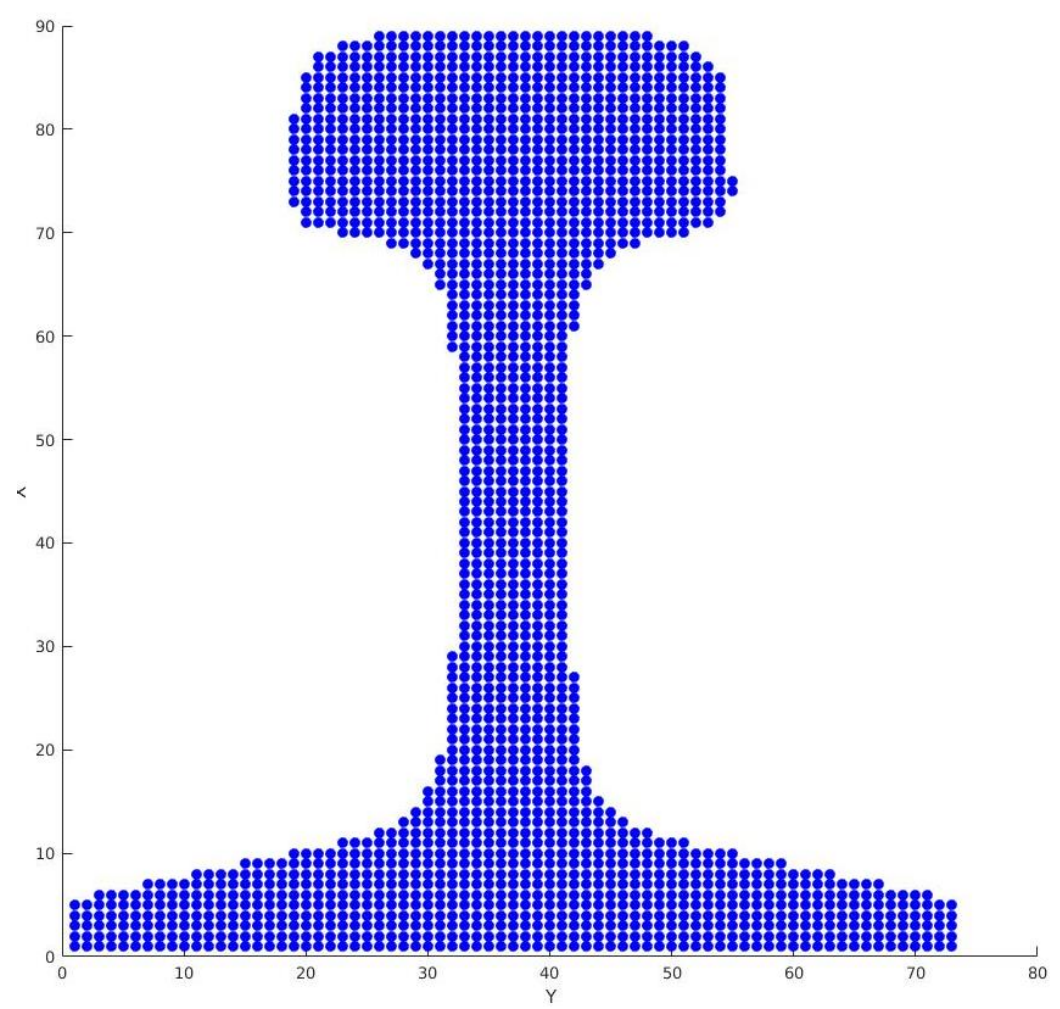

Figure 10: Calibration rail meshing. This coarse mesh is an example of the cubic grid output of the processed STL rail.

Multiple 3-D test cases were solved for the EFIT implementation. In all cases, the output was the magnitude of the velocity vector for each recorded element - again, only the even element positions were used in order to reduce data file size. EFIT Case 1 was a simple rectangular aluminum block with a small void inside it and a round $1 \mathrm{MHz}$ transducer emitting a 5-period sine pulse normal to the top face (in other words, the drive region was defined as a circle at the top of the simulation space with no time delays). In the very simple Case 1, correct wave propagation behavior was observed, including reflections from the void and tip diffraction about the void (Figure 11). 


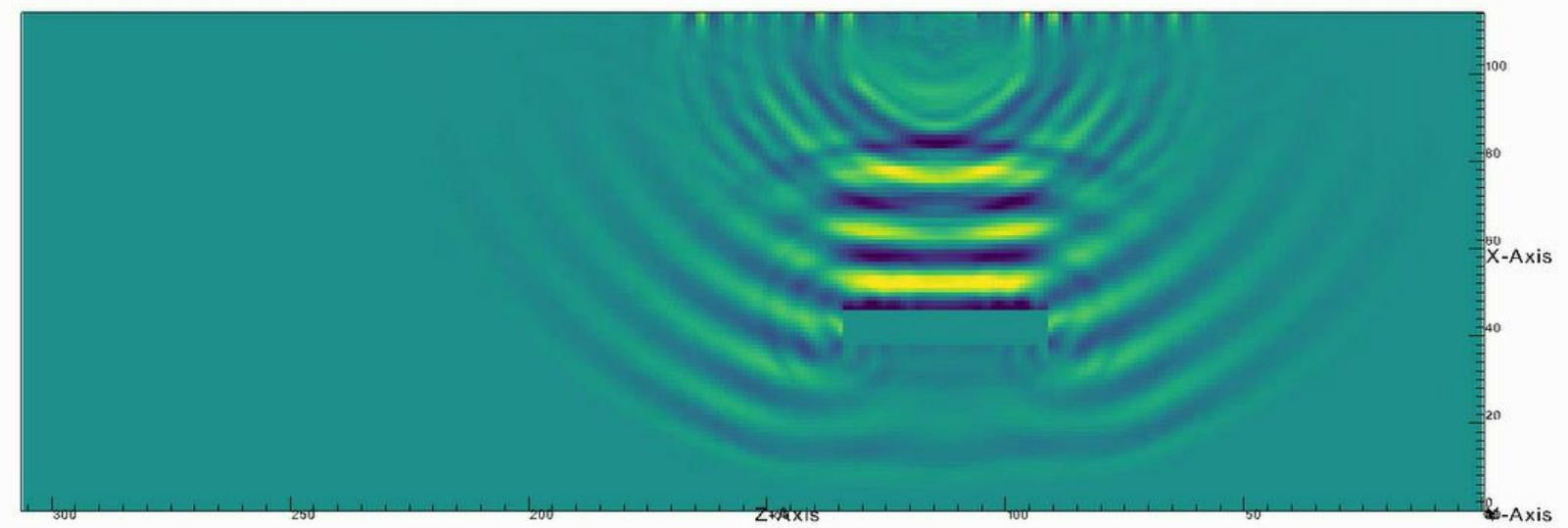

Figure 11: EFIT Case 1-simple block with void. A 2-D slice through the center of the 3-D block allows clear visualization of the wave's interaction with the void. The tip diffraction around the edges of the void are an expected physical response to this ultrasonic pulse.

EFIT Case 2 was a smaller rectangular aluminum block (50x50x100mm) with no inclusions. The transducer was a 1.5 MHz source with a $21.4 \mathrm{~mm}$ radius located at the "top" of the block emitting a 1-period sine pulse (Figure 12). Case 2 includes three separate simulations with the only difference being the angle of propagation produced by the time delay beam steering: $0^{\circ}, 37.5^{\circ}$, and $70^{\circ}$. This comparison validates the methodology used to produce angled beams in the test material. In both angle beam simulations, a leading longitudinal wave is expected to travel at $6,235 \mathrm{~m} / \mathrm{s}$ in the desired direction, while a slower shear wave is expected to travel at 3,139 m/s at a shallower angle. In all three simulations, surface (Rayleigh) waves are expected to travel at $2,906 \mathrm{~m} / \mathrm{s}$ along the plane in which the transducer is located [22]. Using a rough, image-based interpolation technique, the actual propagation speed of the longitudinal wave in the $70^{\circ}$ simulation is $5,963 \mathrm{~m} / \mathrm{s}$. The calculated shear wave speed is $2,981 \mathrm{~m} / \mathrm{s}$ and the calculated surface wave speed is $2,793 \mathrm{~m} / \mathrm{s}$. All of these are within $4-5 \%$ of expected values, which is acceptable given the approximate method used to measure them. 

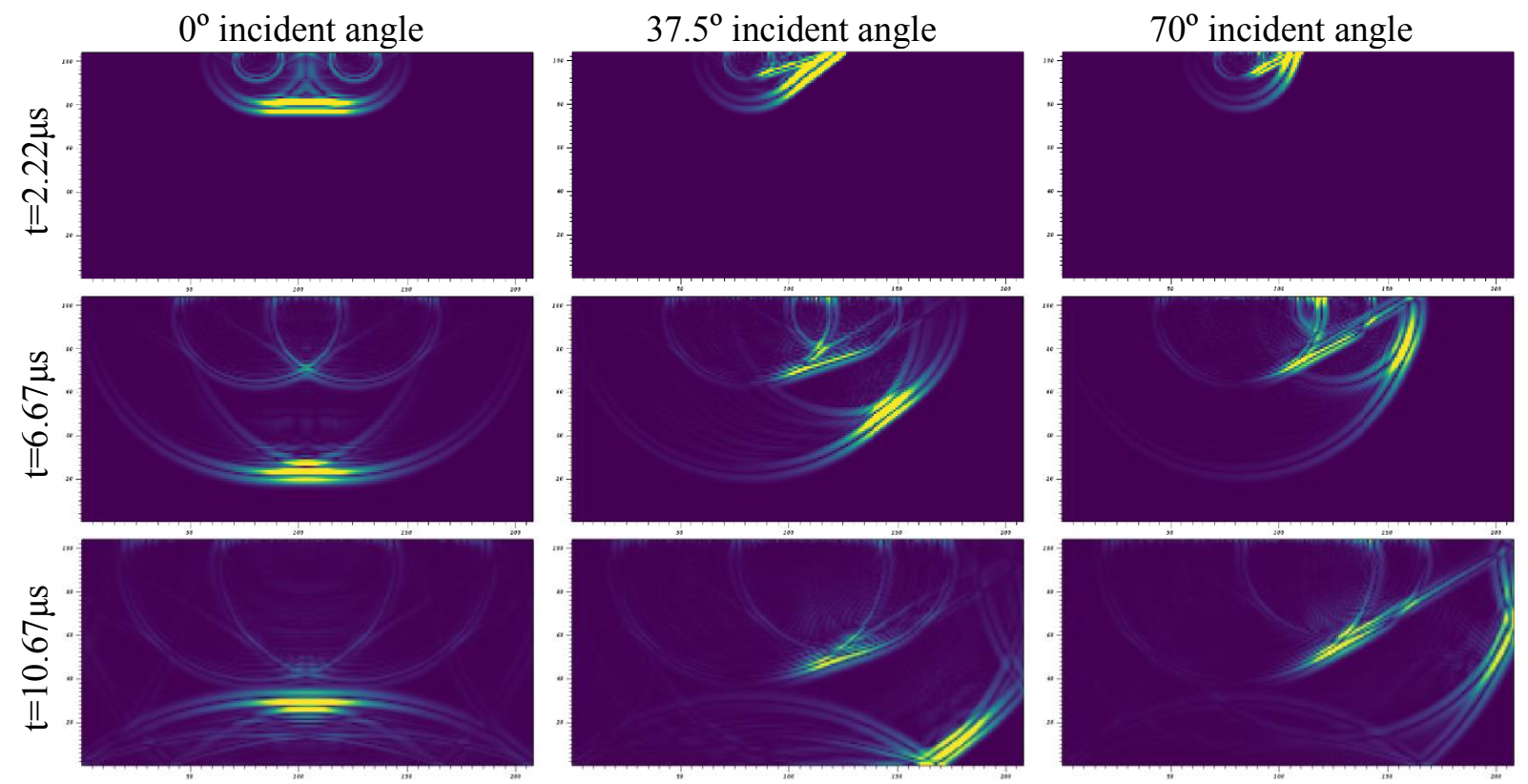

Figure 12: EFIT Case 2 - simple block comparison (no void). The same $1.5 \mathrm{MHz}$ transducer was applied to the same solid rectangular block three times at different angles: $0^{\circ}$ from normal (left), $37.5^{\circ}$ from normal (center), and $70^{\circ}$ from normal (right). The 2-D slice is taken at the center of the 3-D block.

Now that the basic simulation functions are validated, complex simulations in arbitrary geometry can be performed - for EFIT Cases 3-6, that geometry is a 12-inch-long section of steel railroad track. Processing this geometry in MATLAB with a spatial discretization of $0.212 \mathrm{~mm} /$ element takes several hours since it can only be done by a single processor core. Fortunately, the processed geometry file can be used for multiple test cases if the transducer definition text file is altered manually. The executable phase of the simulation takes about 177 minutes on 8 processor nodes, or 111 minutes on 12 processor nodes. During executable initialization, RAM usage climbs to $\sim 120 \mathrm{~GB}$, then levels out at $\sim 85 \mathrm{~GB}$ for the majority of the simulation. The output binaries, again only using data from even element positions, are 885.4 MB each - Cases 3-6 each produced 300 output files, taking up 266.5 GB of storage per simulation.

EFIT Case 3 is a $0^{\circ}, 3 / 4-i n c h, 2.25 \mathrm{MHz} 1$-period sine pulse at the top center of the rail (Figure 14). The ultrasonic wave reflects from the surface of a flat-bottom hole drilled in the 
bottom of the rail, traveling back to the same location where the pulse was emitted. In applied UT testing, the pulse will continue up into the wheel probe, where it will excite the transducer and register a premature reflection in the UT software. In this simulation, the length of time for the wave to reach the hole was used to calculate the simulated propagation speed, which matched exactly the longitudinal speed of sound used in the original simulation setup. This is a good indicator that the simulation matches the physics.

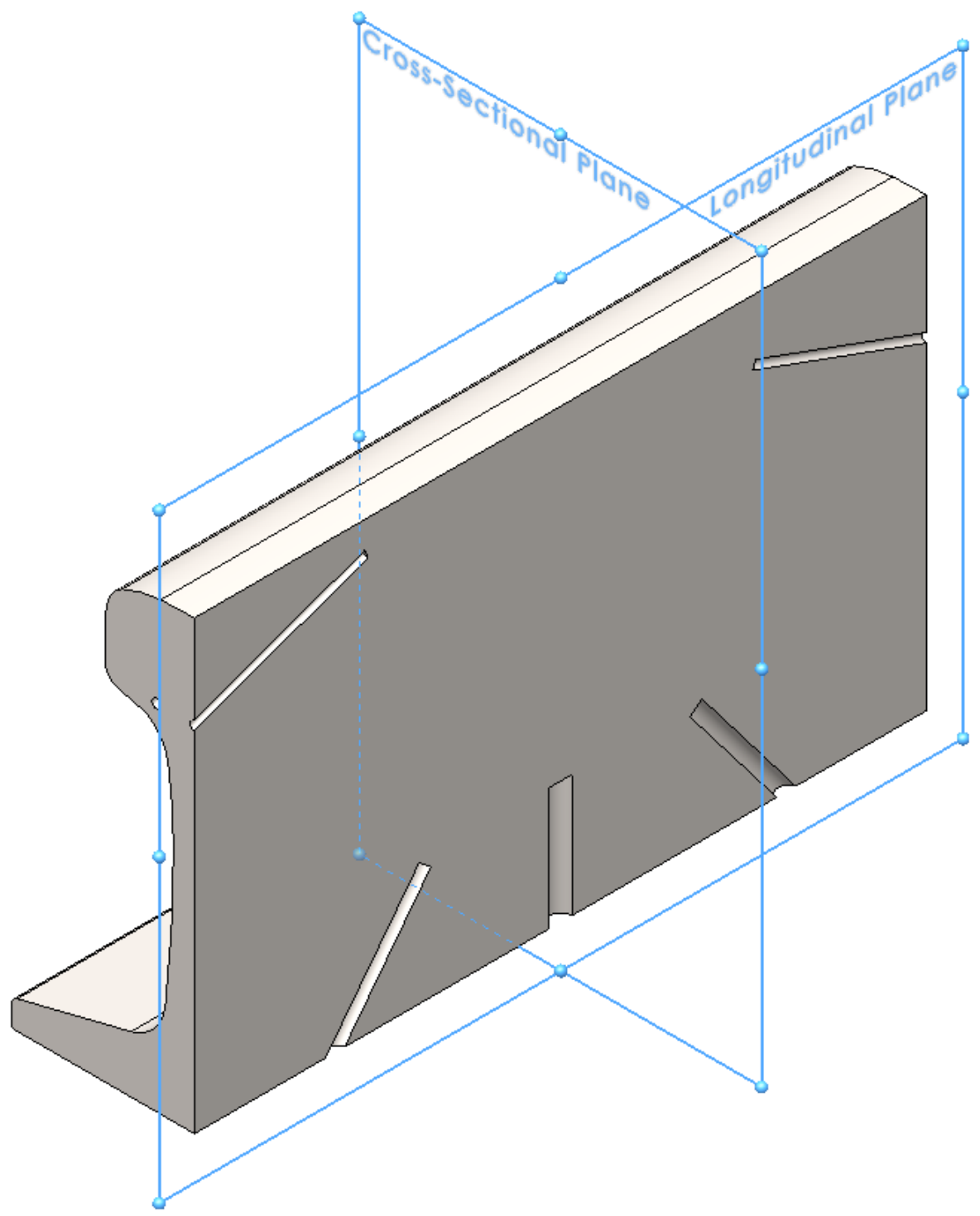

Figure 13: Cutting planes for 2-D slices of 3-D rail space. The cross-sectional view cuts across the rail, while the longitudinal view slices down the length of the rail. Model shown with longitudinal slice active. 

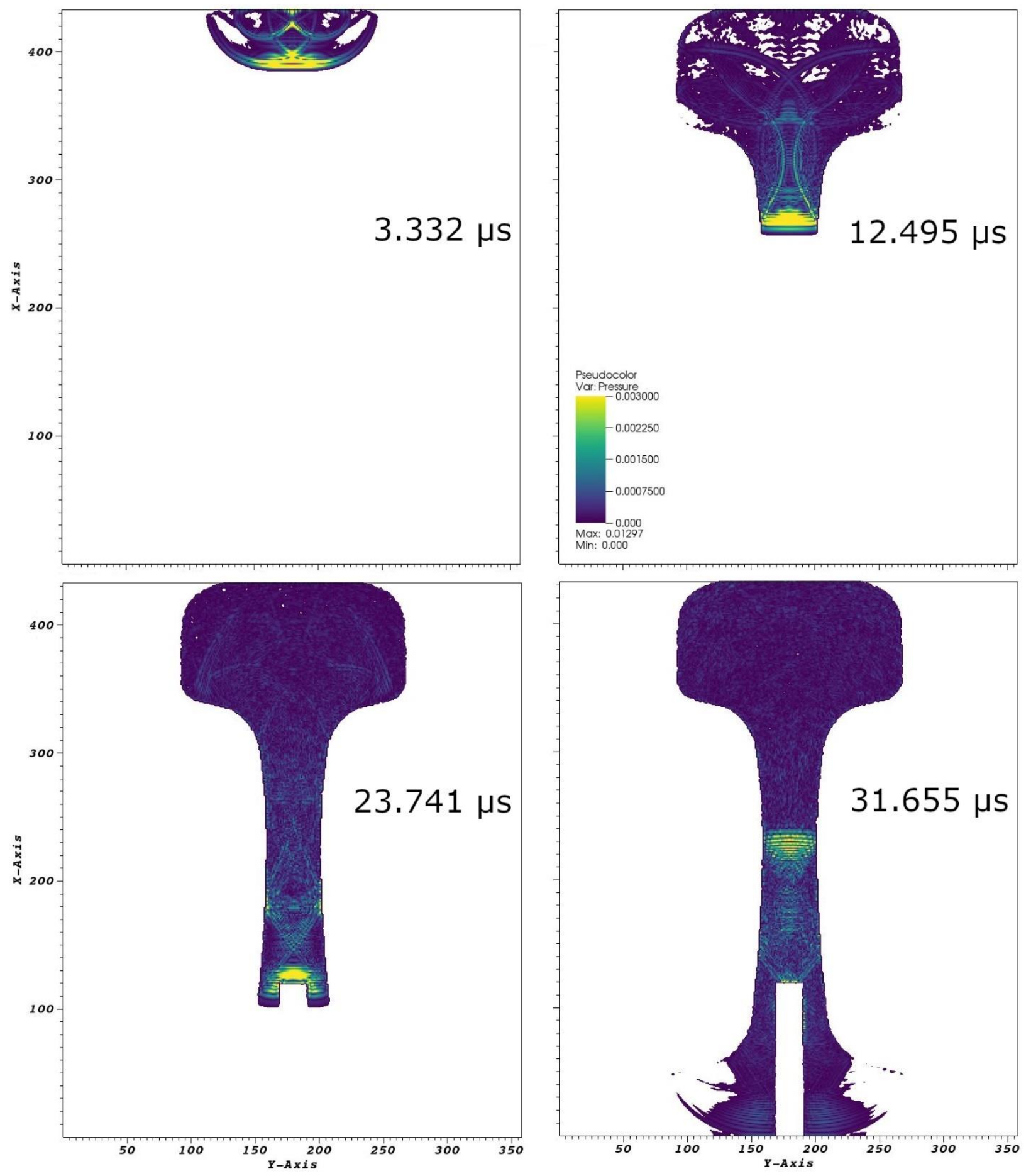

Figure 14: EFIT Case 3 - beam in rail, 0', 2-D slices. The ultrasonic wave enters through the head, travels straight down through the web, reflects from the flat-bottom hole drilled into the bottom of the rail, and passes back up to the pulsing transducer. 


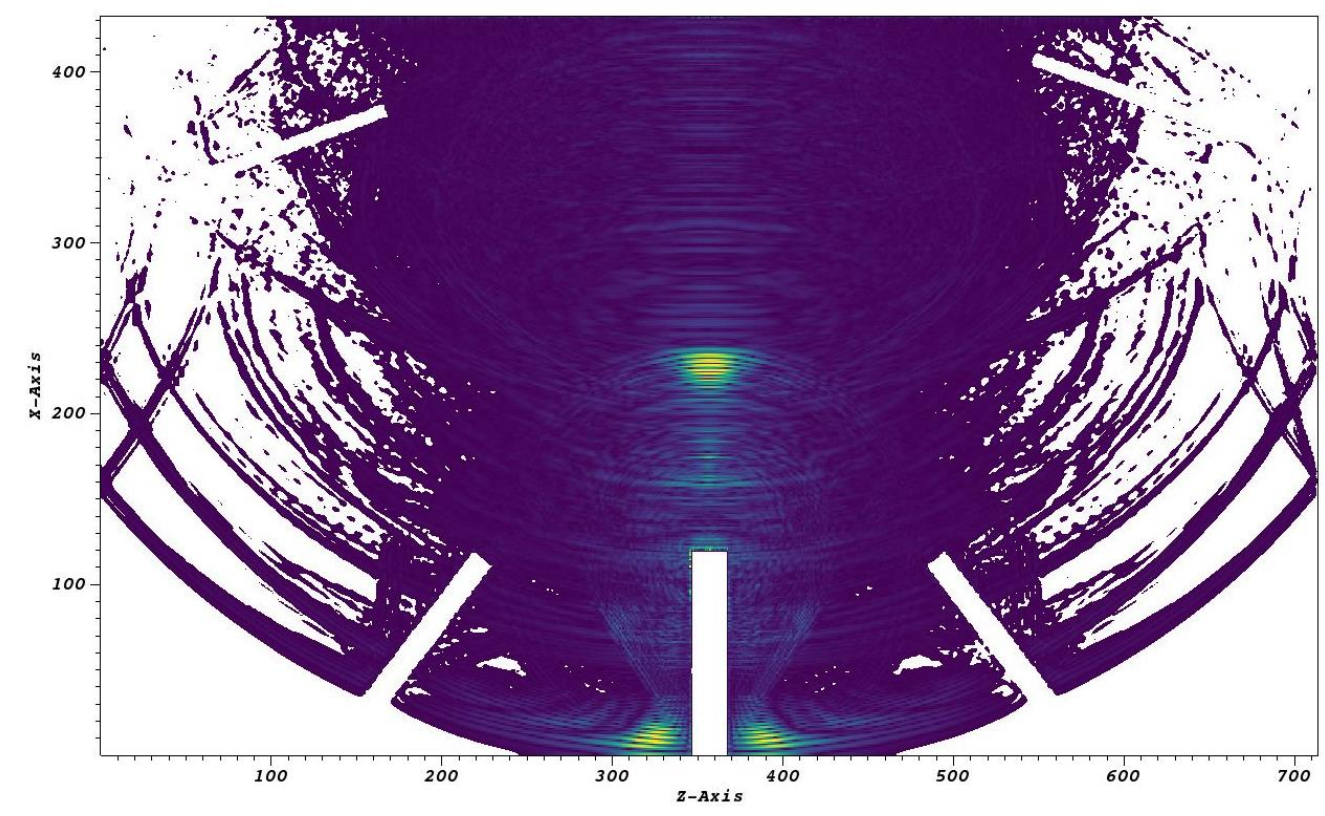

Figure 15: EFIT Case 3 - beam in rail, $0^{\circ}, 2-D$ slice (longitudinal). In this view, the rail is sliced in half lengthwise at $t=31.655 \mu \mathrm{s}$. The pulse can be seen reflecting from the flat-bottom hole. Other drilled holes in the rail are seen outlined by the outer edges of the wave.

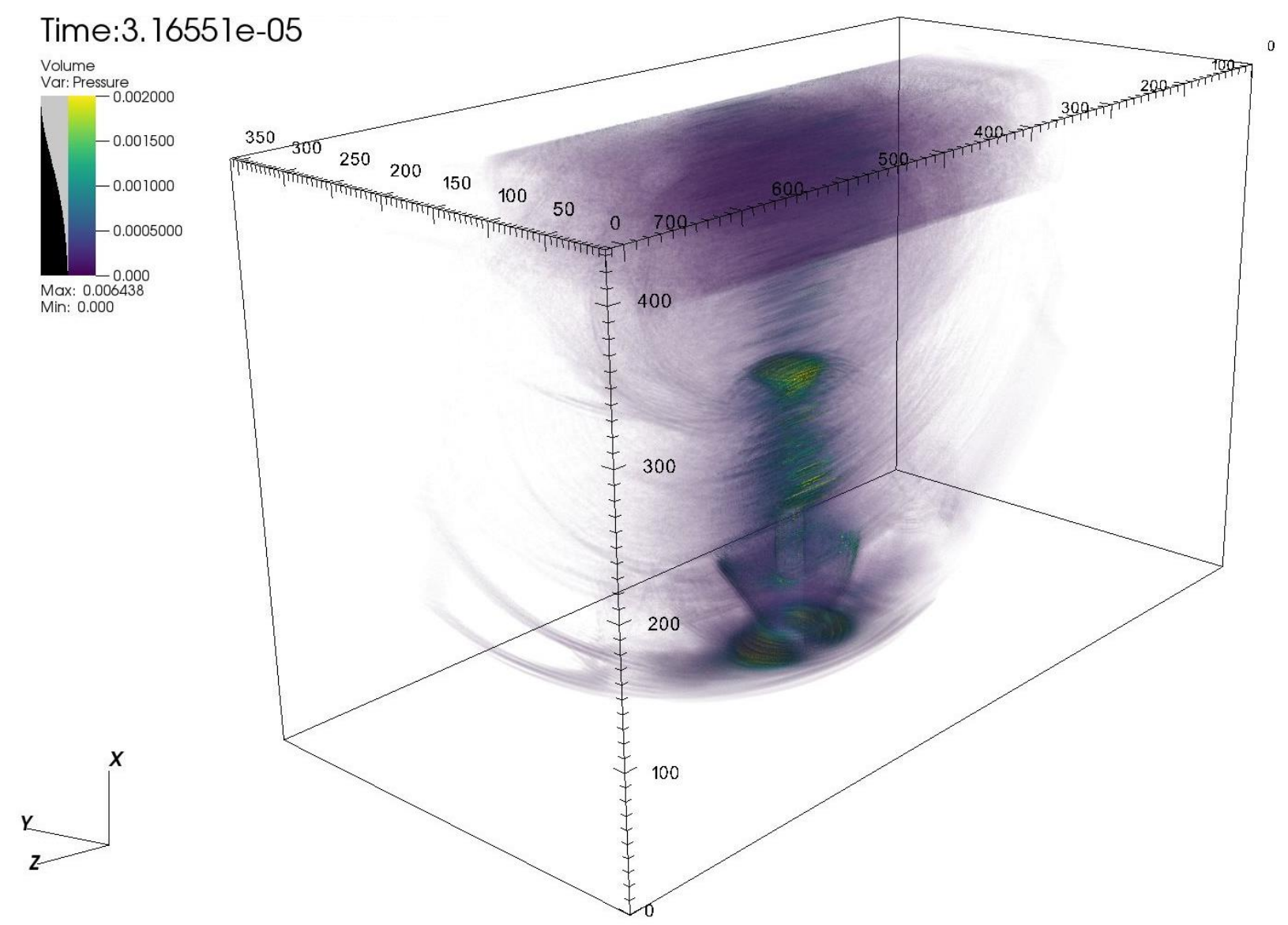

Figure 16: EFIT Case 3 - beam in rail, 0', 3-D view. The head of the rail can be seen slowly filling with scattered reflections from the main wave's path. 
EFIT Cases 4-6 are various angle beams which must be interpreted with an important caveat. To understand that caveat, we must first understand mode conversion and refraction. When an incident longitudinal wave passes between media with different sound speeds (more generally, with different characteristic impedances) at an angle, several things happen. First, a longitudinal wave propagates into the second medium at a refracted angle determined by the ratio of the sound speeds. Depending on the incident angle a shear wave may also be created and propagate in the second medium at a different angle. Since the shear wave speed is always less than the longitudinal wave speed, this angle is always less than the longitudinal wave (Figure 17).

This incident angle at which the refracted longitudinal wave becomes greater than $90^{\circ}$ and only the shear wave propagates in the medium is known as the first critical angle, and the incident angle at which the shear wave no longer propagates is defined as the second critical angle. For incident angles beyond this, surface waves may be created [1]. In applied UT, most angle beams are positioned past the first critical angle so that only the shear wave propagates in the material under test to simplify signal interpretation. Unfortunately, the time delay beam steering method cannot create an input wave beyond the first critical angle. This means that the results for EFIT Cases 4-6 are not truly representative of the application; in real testing, only a shear wave would be present, and not a shear wave and a longitudinal wave. However, the results are no less physical because of this limitation - if a shallower incident angle was used in the UT wheel probe, it would create the waves shown in the following results. 


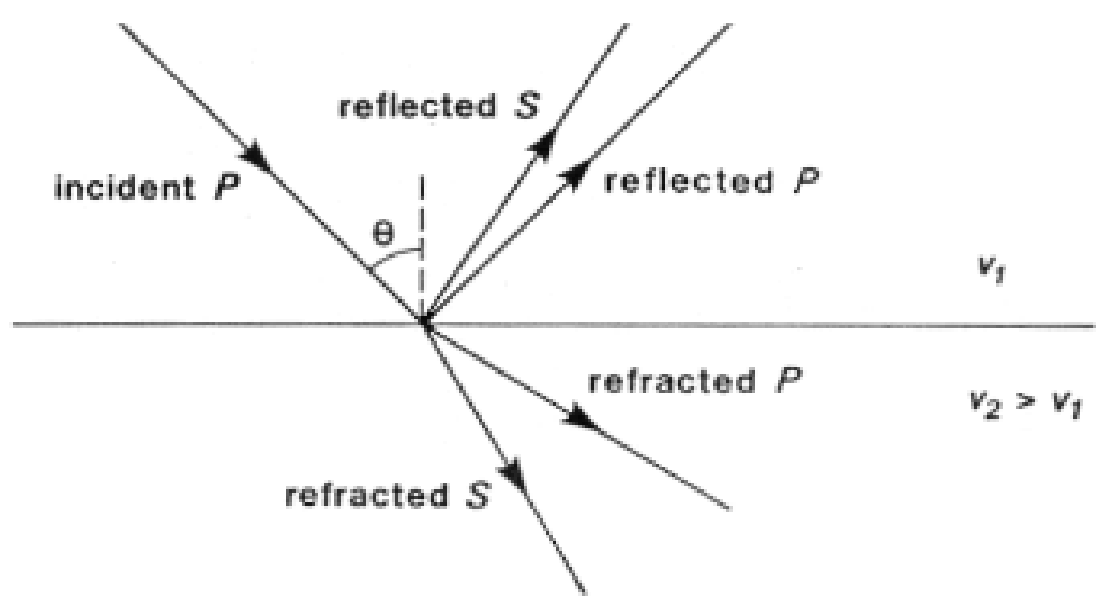

Figure 17: Snell's Law for acoustics in two solids. The angles of the incident longitudinal $(P)$ wave and the reflected $P$ wave are the same. The angle of the reflected shear $(S)$ wave is determined by a ratio of the shear and longitudinal acoustic velocities of Medium 1 (upper material). The angles of the refracted $P$ and $S$ waves are determined by the ratios of acoustic velocities of Medium 1 and Medium 2. From [23].

The transducer for each of EFIT Cases 4-6 is a $3 / 4-i n c h, 2.25 \mathrm{MHz} 1$-period sine pulse at the top of the rail with an offset to align with the appropriate drilled hole. EFIT Case 4 is angled at $56.4^{\circ}$, Case 5 at $37.5^{\circ}$, and Case 6 at $70^{\circ}$ from normal. In each of these simulations, a longitudinal wave can be seen reflecting from the aligned flat-bottom hole, while a shear wave propagates slower and at a smaller angle (Figure 19-Figure 23).

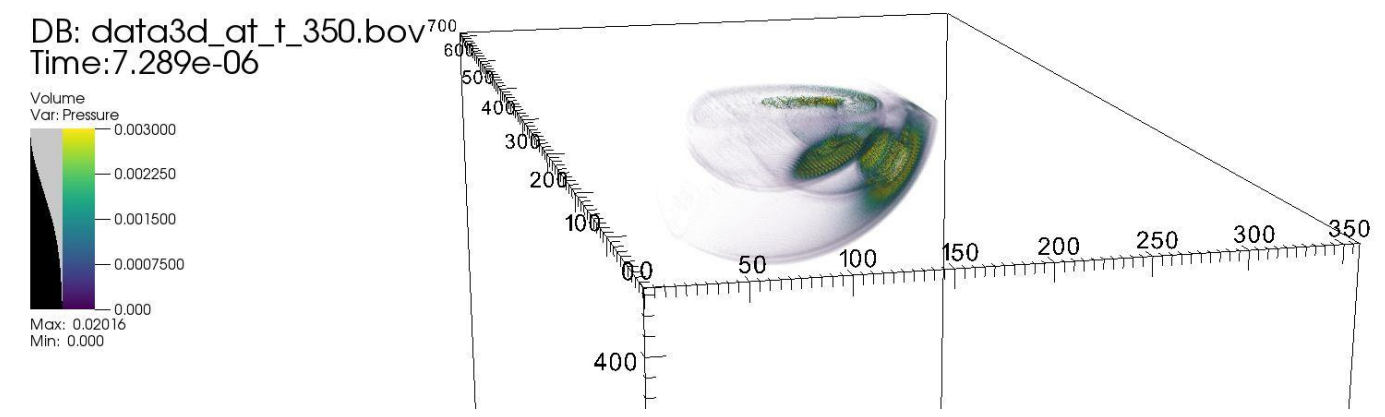

Figure 18: EFIT Case 4-beam in rail, 56.4, 3-D view. Though the center of the longitudinal wave is reflecting from the flat-bottom hole, the outer region of the wavefront continues propagating past the hole. 


\section{DB: data3d_at_†_350.bov Time:7.289e-06}

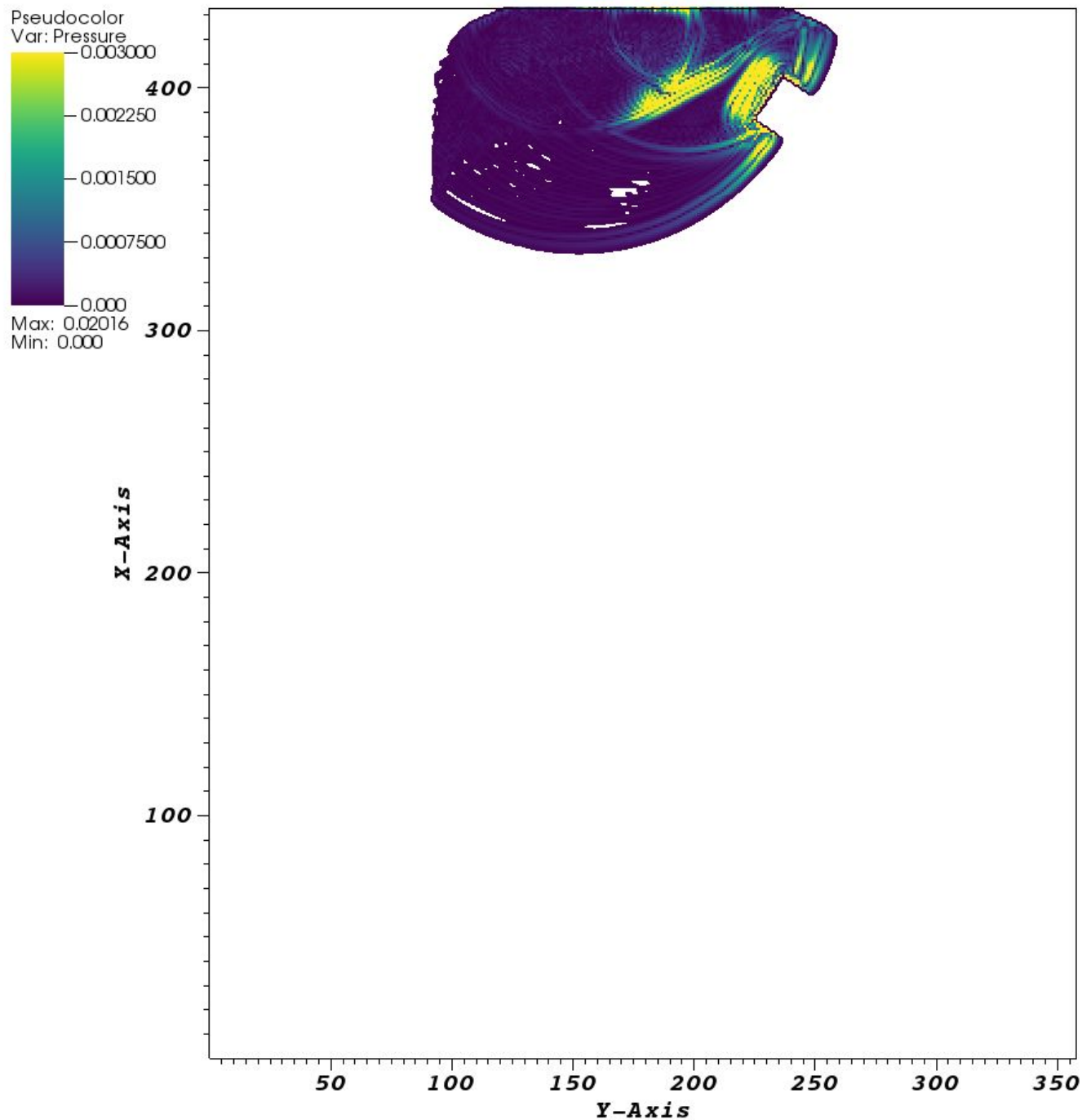

Figure 19: EFIT Case 4 - beam in rail, 56.4, 2-D slice. The longitudinal wave can be seen reflecting directly from an angled flat-bottom hole drilled in the rail head. This transducer is used in practice to inspect the sides of the rail head for near-vertical defects. 


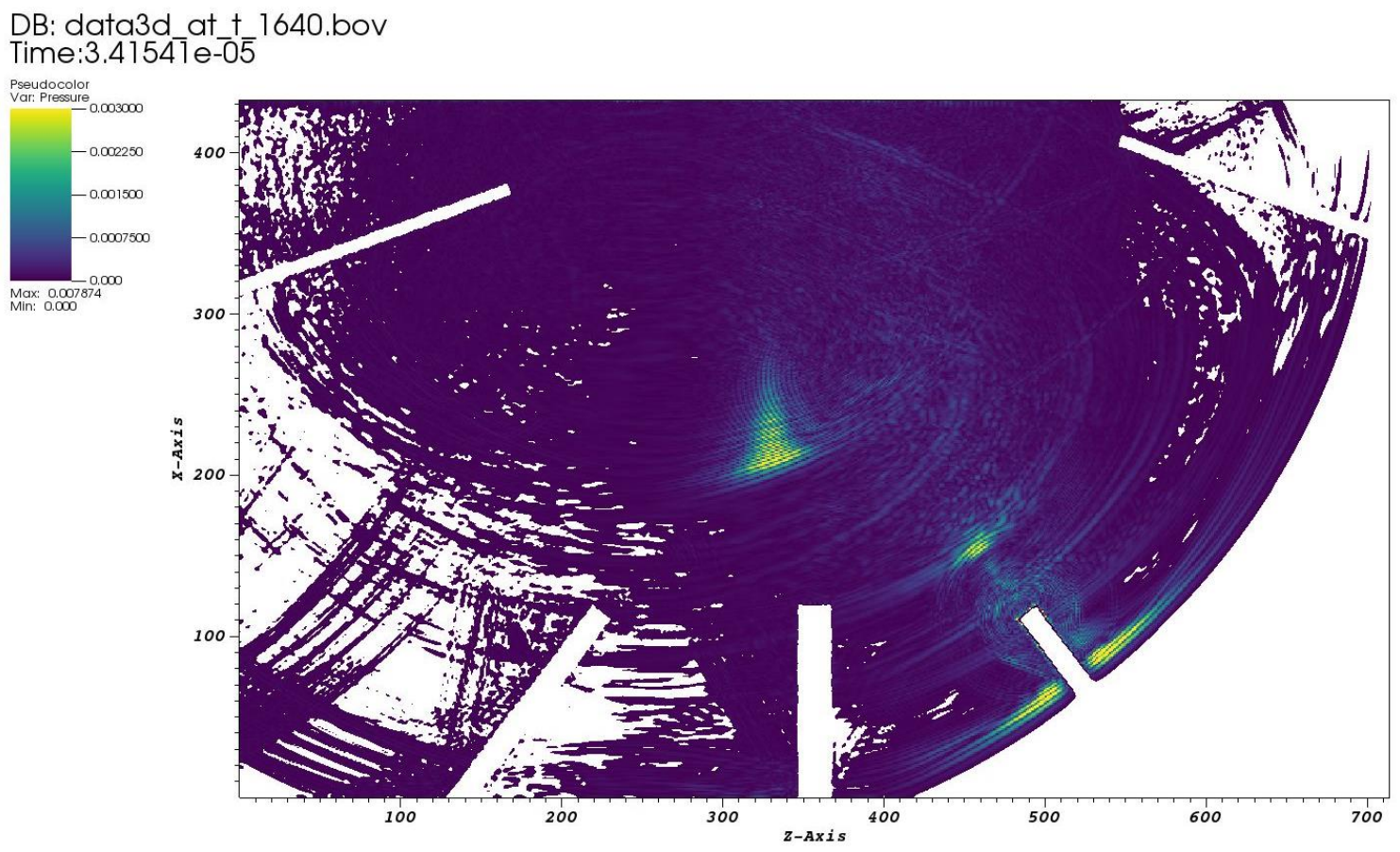

Figure 20: EFIT Case 5 - beam in rail, 37.5', 2-D slice (longitudinal). The longitudinal wave can be seen reflecting from a long, narrow flat-bottom hole drilled at an angle into the bottom of the rail. By happenstance, the shear wave is propagating directly toward the center-bottom hole.

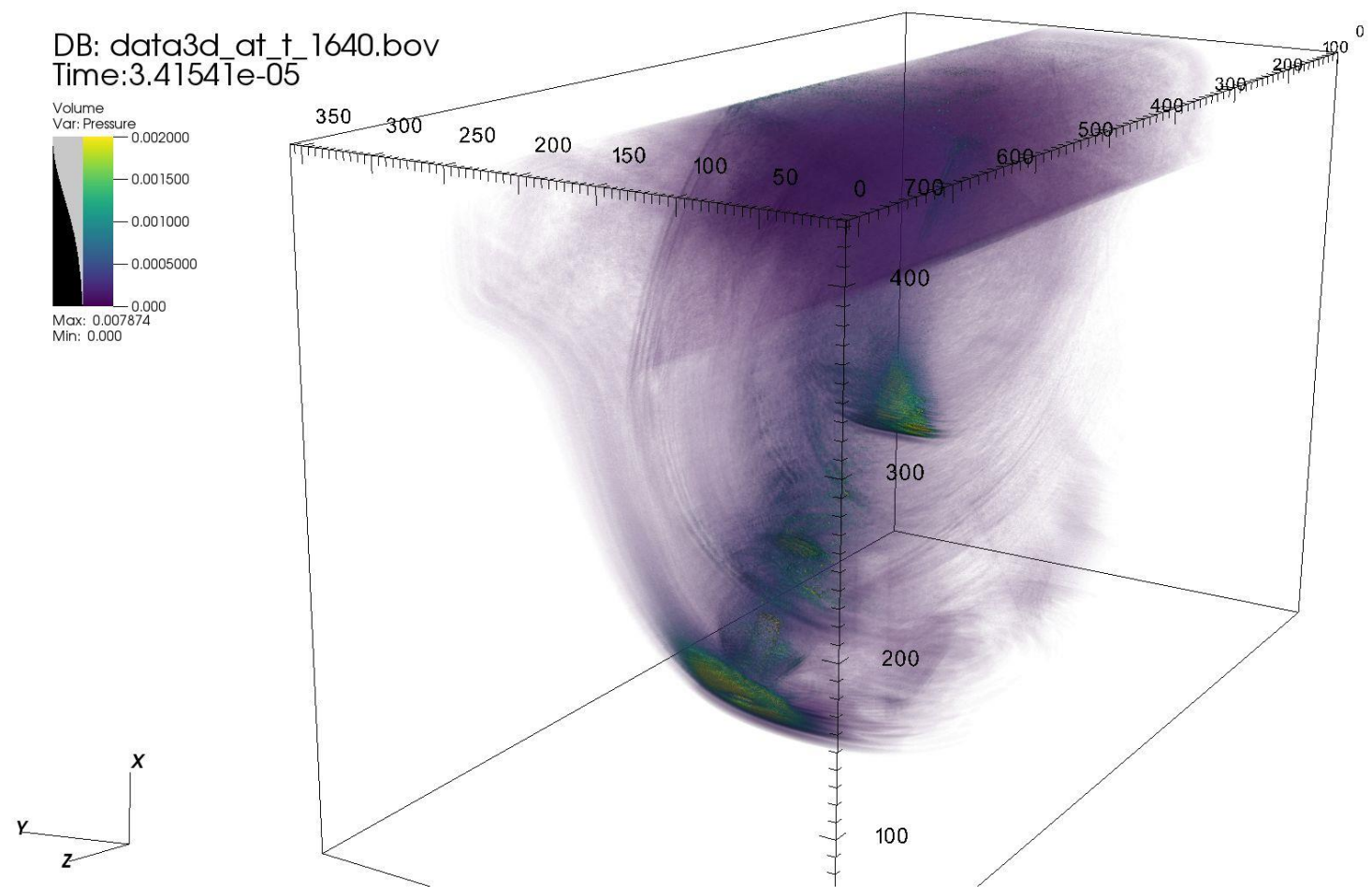

Figure 21: EFIT Case 5 - beam in rail, 37.5', 3-D view. Though the center of the longitudinal wave is reflecting from the flat-bottom hole, the outer region of the wavefront continues propagating past the hole. The low-energy far outer regions of the wavefront can be seen filling the rail with small reverberations. 


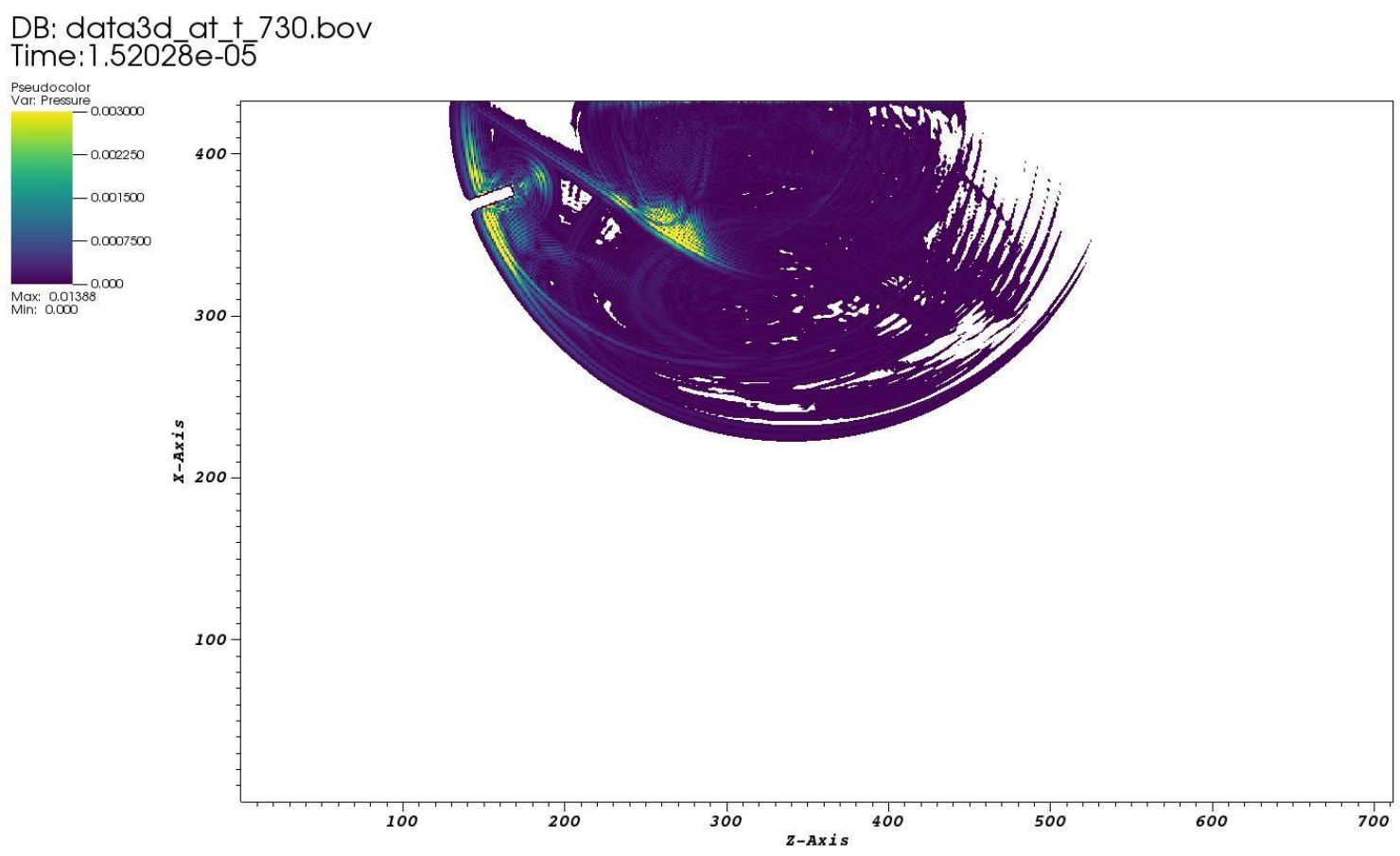

Figure 22: EFIT Case 6-beam in rail, 70', 2-D slice (longitudinal). The longitudinal wave can be seen reflecting from a long, narrow flat-bottom hole drilled at an angle into the end of the rail.

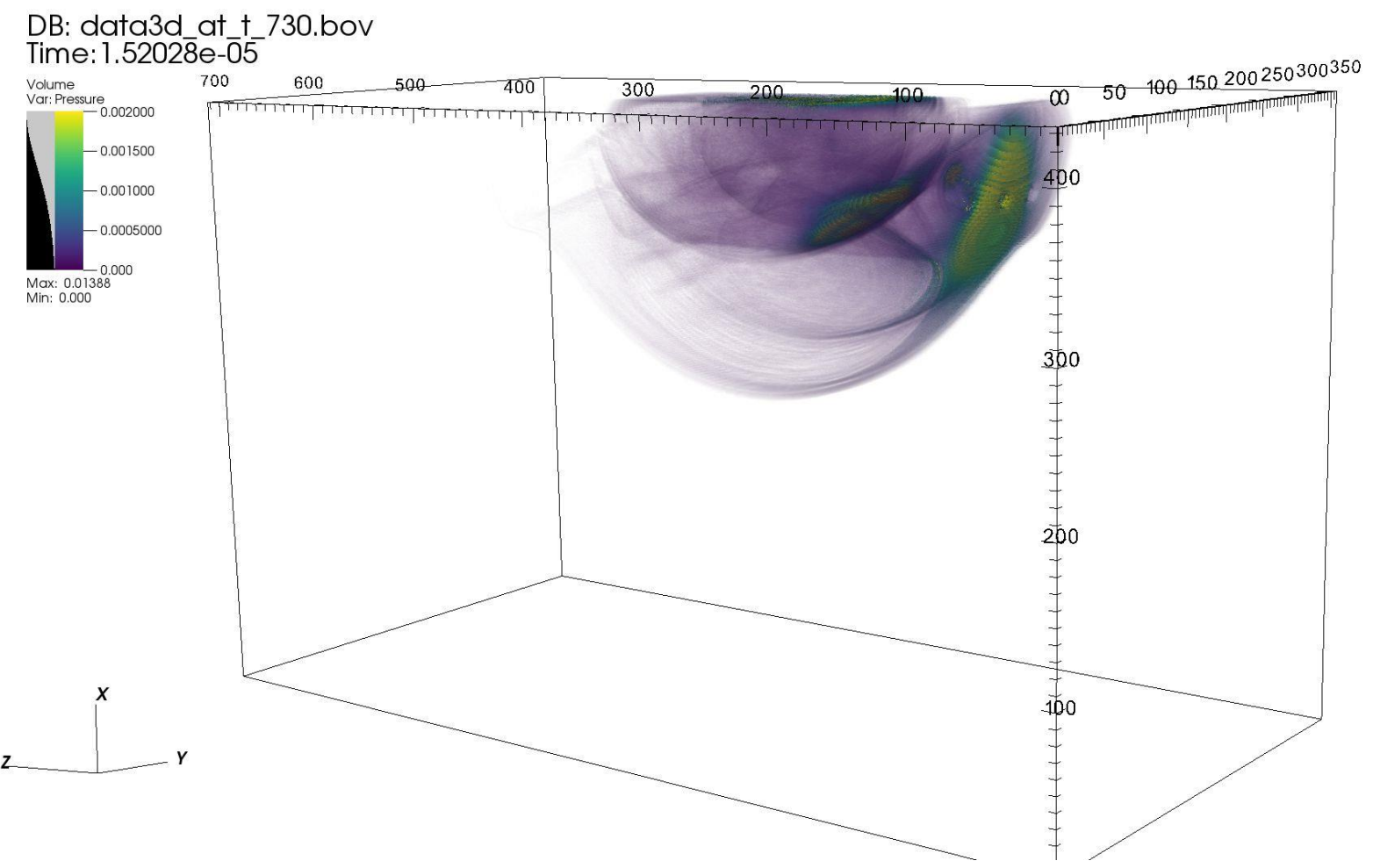

Figure 23: EFIT Case 6- beam in rail, 70', 3-D view. Though the center of the longitudinal wave is reflecting from the flat-bottom hole, the outer region of the wavefront continues propagating past the hole. 


\section{CHAPTER IV: Conclusions and Future Work}

The primary goals of this thesis were achieved: development of a tool for simulating, visualizing, and analyzing ultrasonic wave propagation in arbitrary geometry. However, there are still many opportunities for improvements and further exploration. Two features were implemented in this tool with only partial success, and thus were not included in the results chapter: multi-node transducer definition and transducer curvature mapping. Multi-node transducer definition passes information about transducer definition, drive function, time delays for beam steering, etc. from the processor node which contains the transducer center point to any other nodes that the transducer spans on the spatial domain. The drive function was able to execute correctly, but at times late in the simulation, linear time-growth instability artifacts begin to appear near the nodal boundaries of the transducer space. Very similar instabilities occur when applying transducer curvature mapping, which drops each transducer element "down" from the top of the simulation space until it reaches the top of the material geometry, thus projecting the transducer onto the surface. Both features would be beneficial, but their exclusion can be overcome for many test cases, including those in this thesis. Multi-node transducer definition can be avoided by simply examining the size and location of the transducer and planning the number of processor nodes used accordingly. It limits the speed of the simulation but does not affect the results - and as noted in EFIT Case 3, the longest simulation run time was only 3 hours. Transducer curvature mapping can be avoided by trimming the top of the simulation geometry before exporting it from the modeling software, such that the top surface of the geometry ends up being coplanar with the top face of the simulation space (which is where the transducer is defined, by default). This approach requires a small amount of additional setup work and is a minor approximation but does not significantly impact results for the geometry in EFIT Case 3. 
Other features were not attempted in this implementation that could add considerable value. For instance, a multiphysics AFIT/EFIT simulation with transducers defined within a fluid region would be a more accurate representation of what is physically happening in a UT wheel probe. This would allow multiple angle beams to be focused on one area of the fluid-solid boundary, enabling simulation of the interactions of several ultrasonic beams fired at the same time. One of the largest limitations that would be eliminated is the lack of true refraction and mode conversion in the current implementation. Additionally, this method would be ideal for defining transducers at arbitrary locations and angles, eliminating the need to use time delay beam steering.

Beyond adding features, an important step would be performing experimental validation of the simulation results. For the tool to be truly useful as a development aid, the results must be tested against real-world experiments to build confidence in the implementation. This could be done first for very simple setups like EFIT Case 2 to prove the basic functionality, then expanded to more complex tests like EFIT Cases 3-6. The input parameter would be recorded at the center of the transducer in the simulation and scaled using the drive function to match the experimental data.

Though the simulation tool has limited applicability to R\&D as it stands, there are many ways it can be used. The visualizations created in VisIt give great clarity to a process that is invisible to human senses - it may add significant value to educational materials on UT and the science of acoustics. In fact, the video results are so intuitive that it is possible to use them as communication tools to explain ultrasonic phenomena to non-technical parties who do not have formal education in the field. Most importantly, though, the code can be used as a basis for future research and exploration in the field of numerical simulation of acoustics. 
APPENDIX 


\section{MATLAB Code: Input File Generation}

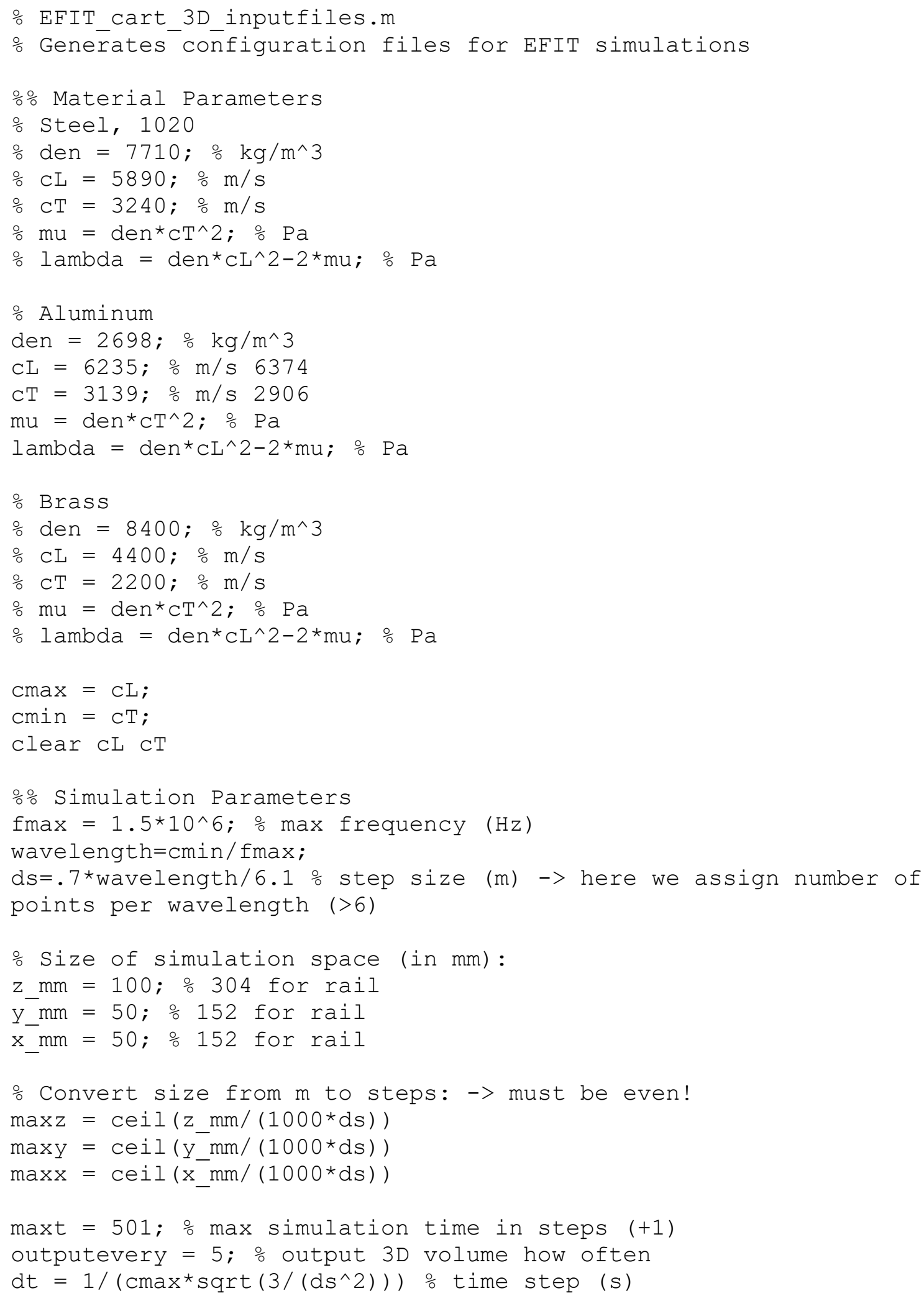


\% Choose a flaw type (everything should be in number of steps)

\% Arbitrary fullspace scatterer:

$\circ \mathrm{nS}=1$

\% numref // numbers scatterers

\% rftype $=[5]$;

o typ // Reflector type: 5 - arbitrary

(fullspace)

$\div \mathrm{nsx1}=[0]$

$\div \mathrm{nsx} 2=[0]$;

$\because \mathrm{ns} \times 3 \mathrm{~s}=[0]$;

ㅇ / / not used

$\div \mathrm{p} 2$ // not used

品 $\mathrm{nse}=[0]$;

o start3 // not used

\% rrad $=\left[\operatorname{maxz}{ }^{\star} \operatorname{maxy}{ }^{\star} \max x\right]$; $\div$ rad // not used

\% rden $=[-1] ; \quad \%$ dd $/ /$ not used

\% rmu $=[-1] ; \quad \% \mathrm{mu} \quad / /$ not used

$\div$ rlambda $=[-1] ; \quad \div$ lambda // not used

\% $\frac{\circ}{\circ}$ inputs: filename, ds, adjustment of origin (xyz in $m$ ), space size (xyz in $m$ )

\% [arbscatt, ] = import_stl_scatterer('Test Block Curved Top x-50 y50 z-100.STL', ds, [0 0 0 $],{ }_{1} x_{-} m m / 1000$ y_mm/1000 z_mm/1000]);

의(size(arbscatt) $\sim=[\operatorname{maxy} \operatorname{maxx} \operatorname{maxz}])$

o disp('Error in Arbitrary scatterer definition. Terminating script.' )

\% return

$\frac{\circ}{0}$ end

Rectangular:

$\mathrm{nS}=0$;

rftype $=[3]$;

Rectangular Prism

nsx1 $1=[\operatorname{round}(25 /(1000 * \mathrm{ds}))]-1 ; \quad \mathrm{p} 1 \quad / /$ z-start (z-end will be

to side of space)

$\mathrm{nsx} 2=[\operatorname{round}(75 /(1000 * \mathrm{ds}))]-1 ; \quad \frac{\mathrm{p} 2}{\mathrm{a}} \mathrm{/} / \mathrm{z}$-end

$\mathrm{nsx} 3 \mathrm{~s}=[\mathrm{round}(10 /(1000 * \mathrm{ds}))]-1 ; \quad$ start $3 / / \mathrm{y}$-start

$\mathrm{ns} \times 3 e=[\operatorname{round}(60 /(1000 * \mathrm{ds}))]-1 ;$ \% end3 // y-end

rrad $=[\operatorname{round}(20 /(1000 * \mathrm{ds}))]-1 ;$ rad $/ / \mathrm{x}$-start

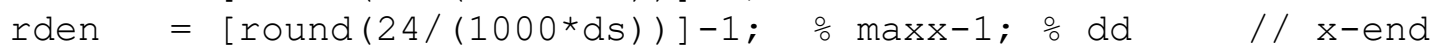

rmu $=[0]$;

rlambda $=[0]$;

\% numref // number of scatterers

이 // Reflector type: 3 - Right

if $($ (nsx $1>n s x 2)$ || (nsx3s>nsx3e) || (rrad>rden))

disp('Error in Rectangular scatterer definition. Terminating

script.')

return

end

응 Transducer info (everything in number of steps)

ntrans $=1$;

transducer_z $=[$ round $(50 /(1000 * d s))]$; $\frac{\circ}{0}$ transducer $z$ position

transducer_y $=[\operatorname{round}(25 /(1000 * d s))]$; $\%$ transducer y position

transducer_x $=[\max x-1]$; $\frac{\circ}{0}$ transducer $\mathrm{x}$ position; $(\max \mathrm{x}-1)$ is

positioned on top

transducer_rad $=[\operatorname{round}(10.7 /(1000 * d s))]$; $\frac{\circ}{0}$ transducer radius 


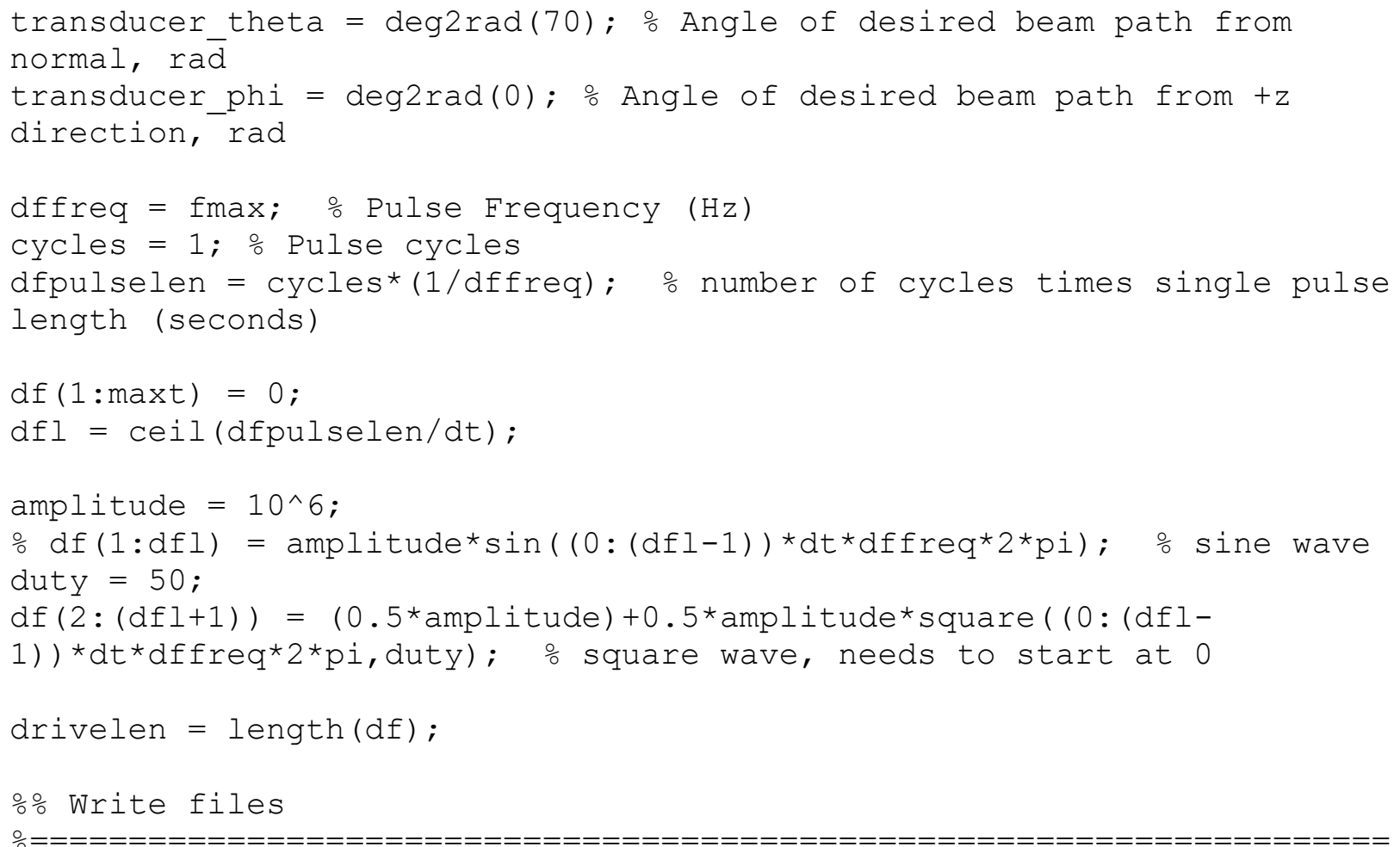

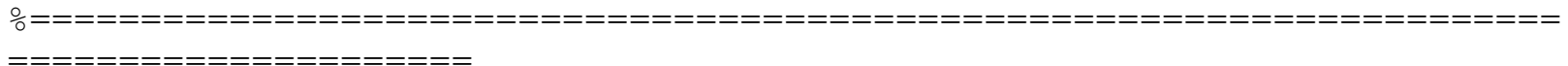

Write Inputfiles for simulation - 응 NOT CHANGE THE ORDER OF THIS PART

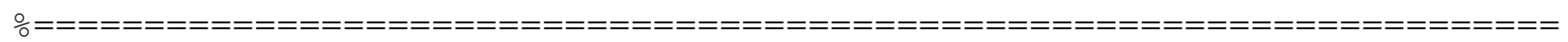
$============$

[fname, pname] = uiputfile('in.file', 'Save Configuration'); fp=fopen ('in.file', 'w');

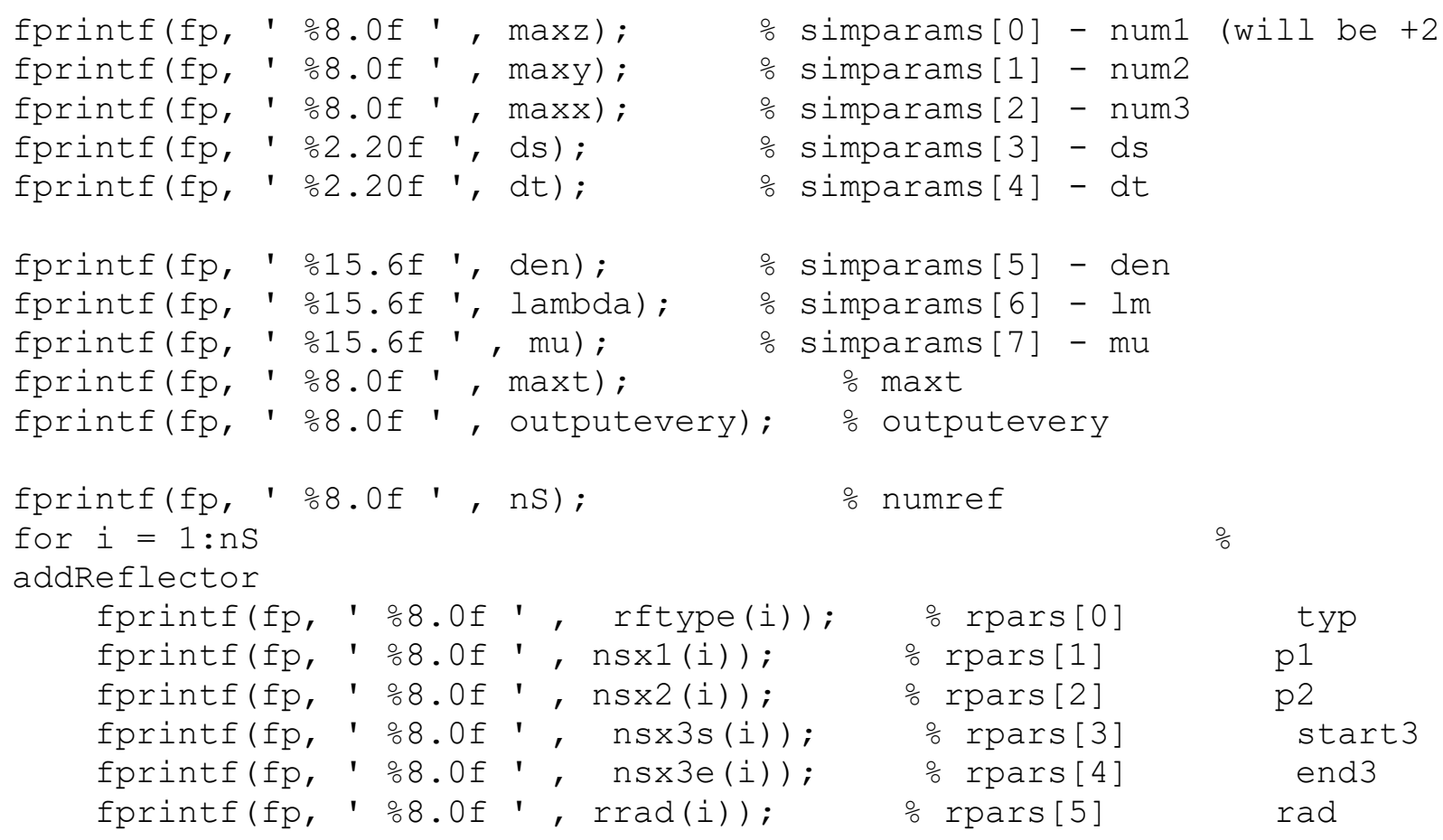




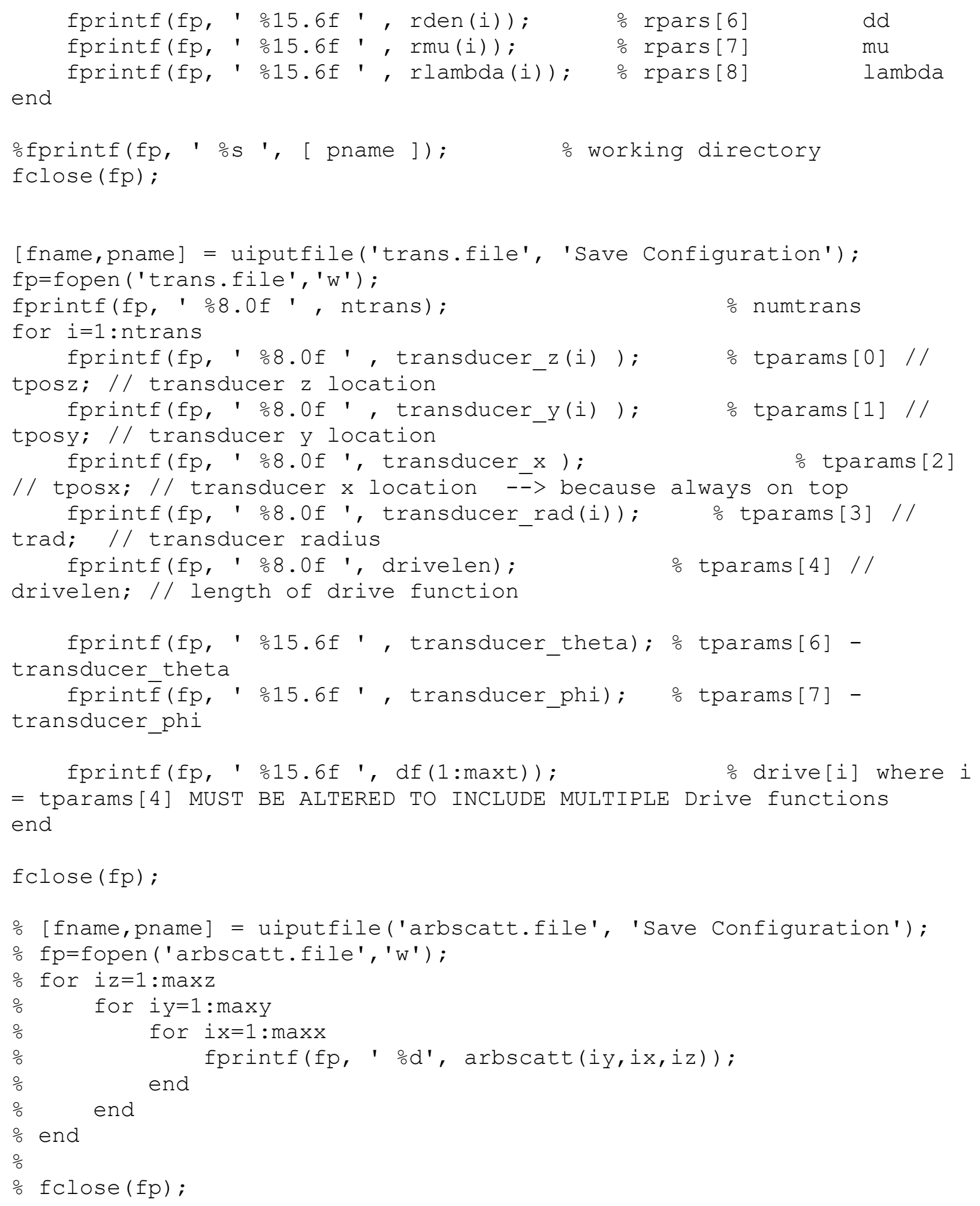




\section{MATLAB Code: Arbitrary Geometry Importing and Processing}

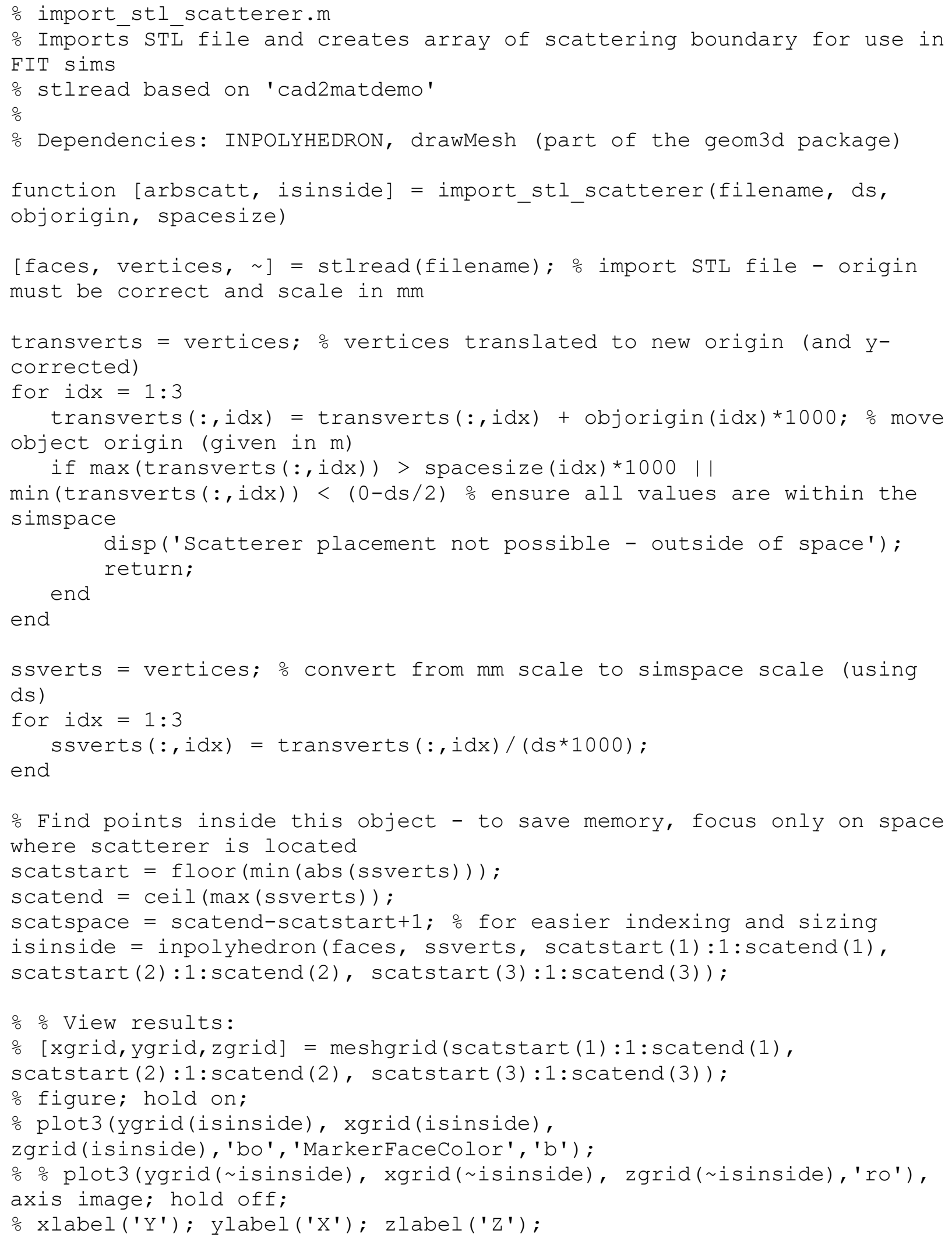


o clear xgrid ygrid zgrid;

o Now create logical array for entire space as input to simulation fullspace $=[$ ceil (spacesize(2)/ds), ceil (spacesize(1)/ds), ceil(spacesize(3)/ds)]; $\frac{\circ}{0}$ size of full space (steps) arbscatt $=$ false (fullspace); $\frac{\circ}{\circ}$ this could get big quickly - use logical to reduce size

o Cuts off last face in each dimension (vertices --> nodes) if $($ size $($ isinside, 2$)==($ scatend $(1)-\operatorname{scatstart}(1)+1) \quad \& \&$ ( $\operatorname{size}($ isinside, 2)-1)==fullspace(2)) \% nothing got switched around, sizes are correct

arbscatt (scatstart (2) +1: scatend (2), scatstart (1) +1: scatend (1), scatstart $(3)+1$ : scatend(3)) = isinside (1: scatspace (2) -1, 1: scatspace (1) $1,1$ : scatspace $(3)-1)$; else end disp('Something wrong with logical array');

\% View results for whole space - MAY BE BIG!!

\% [xgrid,ygrid, zgrid] $=$ meshgrid(1:1:fullspace (2), 1:1:fullspace (1), 1:1:fullspace (3)) ;

o figure; hold on; o plot3 (ygrid(arbscatt), xgrid(arbscatt), zgrid (arbscatt), 'bo', 'MarkerFaceColor', 'b') ; o plot3 (ygrid( arbscatt), xgrid( arbscatt), zgrid( arbscatt), 'ro') ; hold off;

o xlabel('Y'); ylabel('X'); zlabel('Z');

function [fout, vout, cout] = stlread(filename)

o Reads ASCII stl file and returns a vertex list and face list for Matlab patch command

fid=fopen(filename, ' $r$ '); oopen the file, assumes STL ASCII format. if $\mathrm{fid}=-1$

error('File could not be opened, check name or path.')

end

응 files of form:

$\%$

solid BLOCK

o color 1.0001 .0001 .000

o facet

\% normal $0.000000 e+00 \quad 0.000000 e+00-1.000000 e+00$

\% normal $0.000000 e+000.000000 e+00-1.000000 e+00$

\% normal $0.000000 e+000.000000 e+00-1.000000 e+00$

o outer loop

$\div \quad$ vertex $5.000000 e-01-5.000000 e-01-5.000000 e-01$

\% vertex $-5.000000 e-01-5.000000 e-01-5.000000 e-01$

$\div \quad$ vertex $-5.000000 e-015.000000 e-01-5.000000 e-01$

endloop 


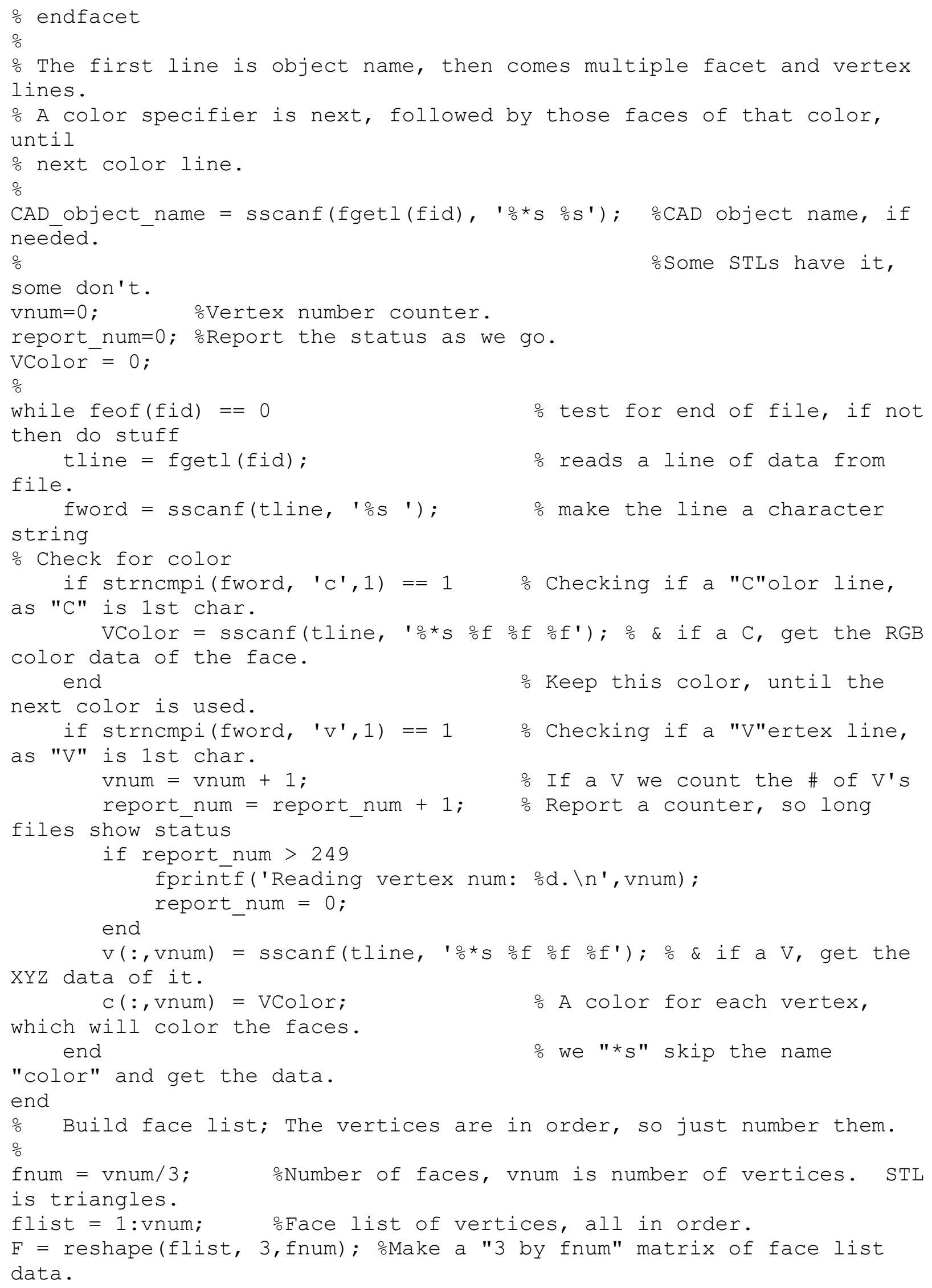




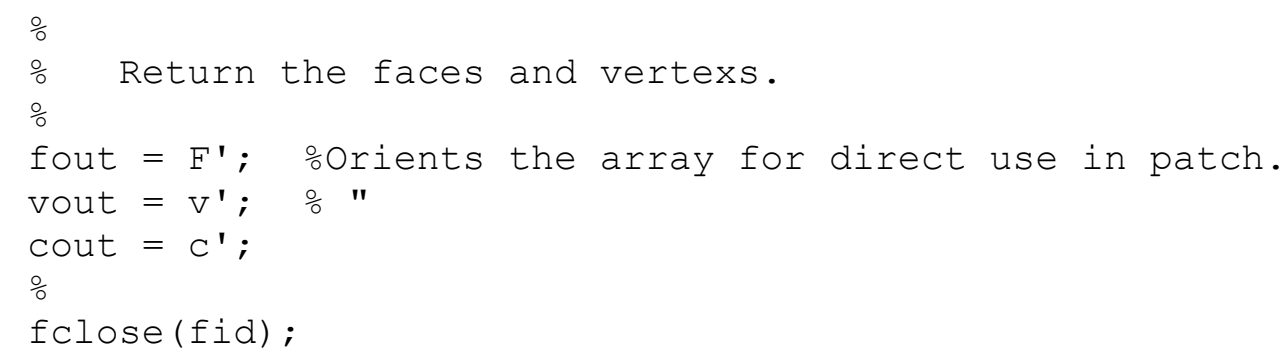




\section{C++ Code: 'efit.cpp'}

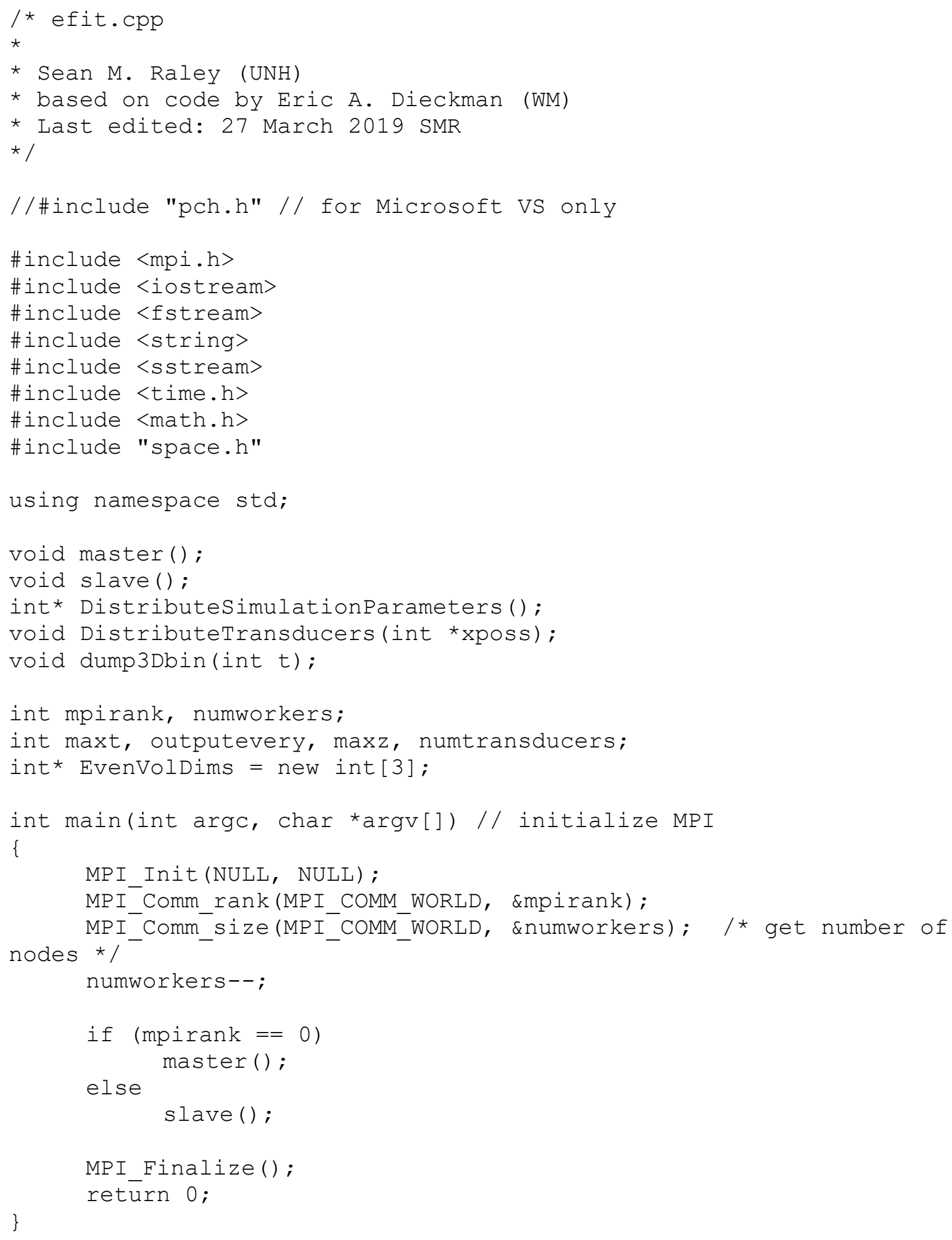




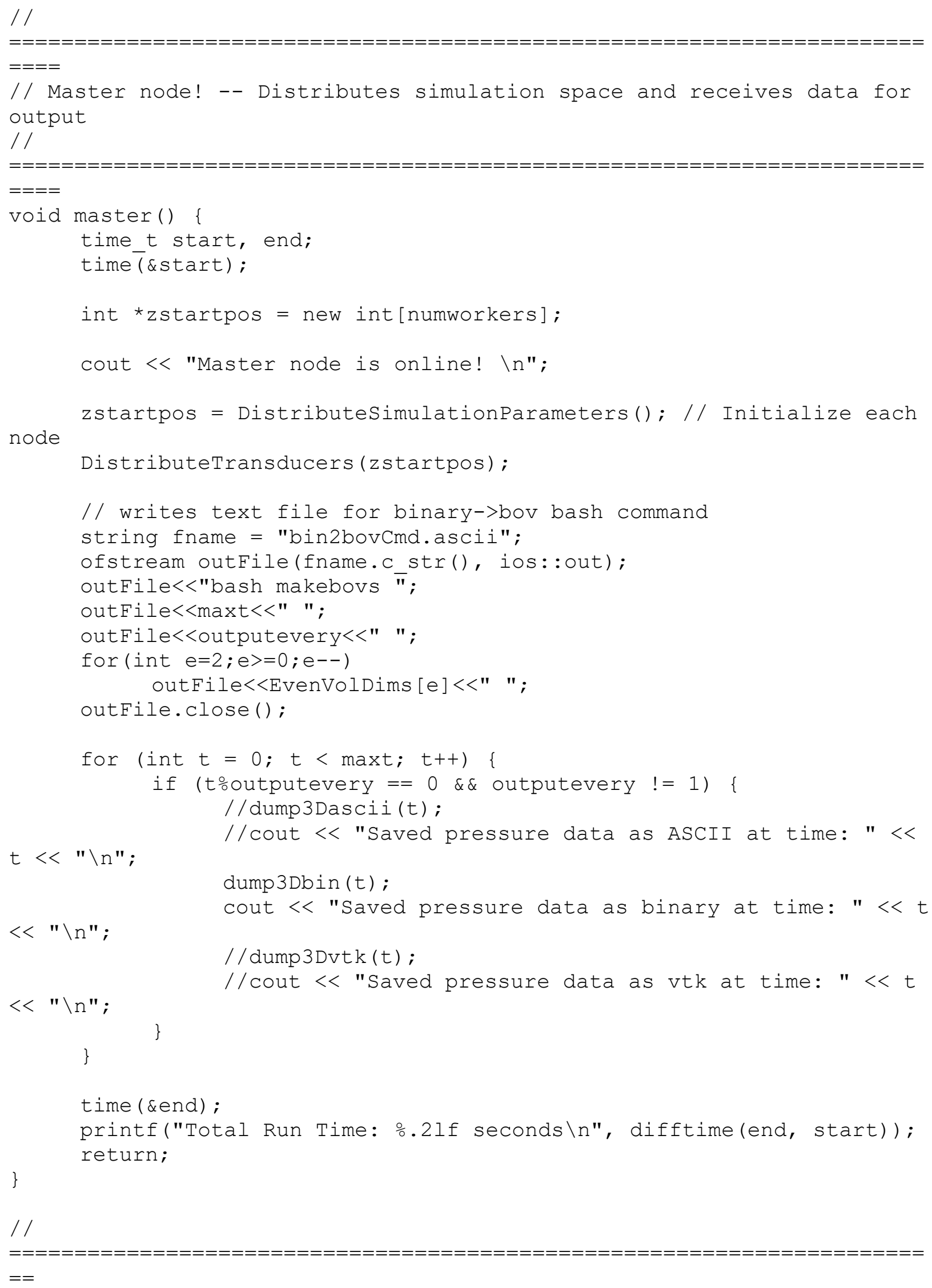


// Slave node! -- Does the grunt work

//

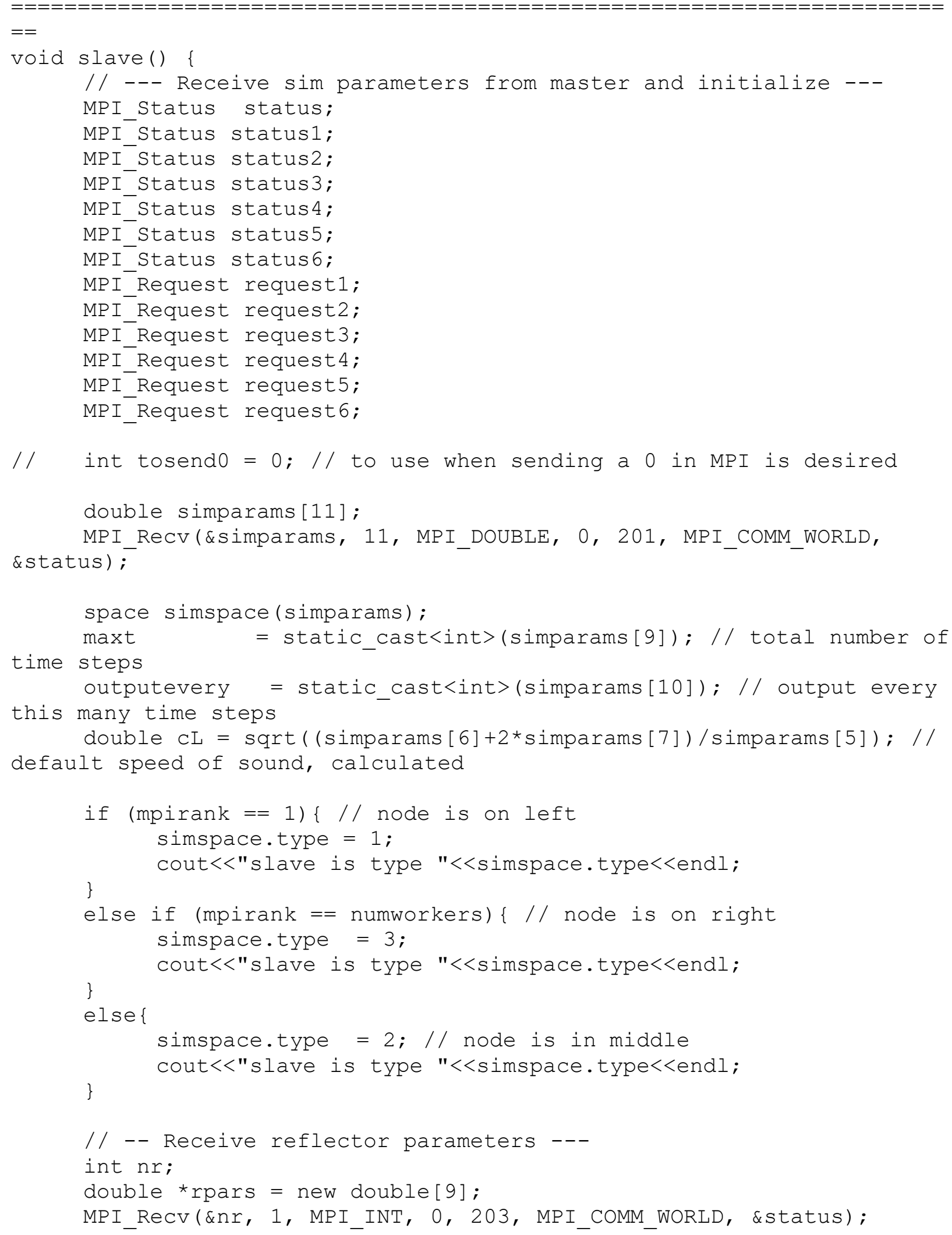




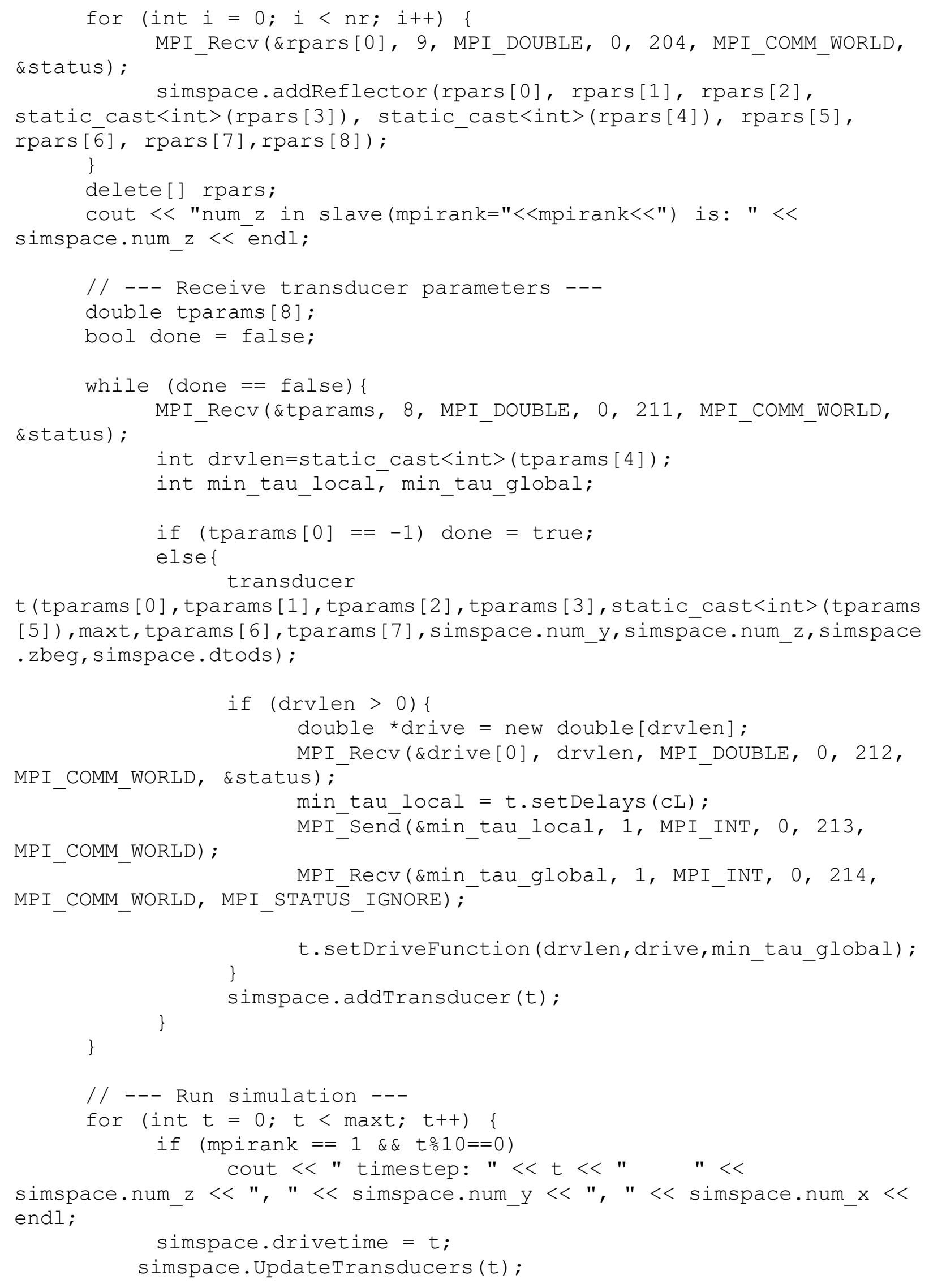


if ( $t$ ooutputevery $==0) \quad\{/ /$ sends output to master node int len = simspace.GetEvenVolLen (); // get even num_z length for each node double* $\mathrm{x}=$ simspace.GetEvenVol (len);

MPI_Send ( $\& l e n, 1$, MPI_INT, 0, 1101, MPI_COMM_WORLD) ; MPI_COMM_WORLD) ; MPI_Send (\&x[0], len, MPI_DOUBLE, $0,110 \overline{2}$,

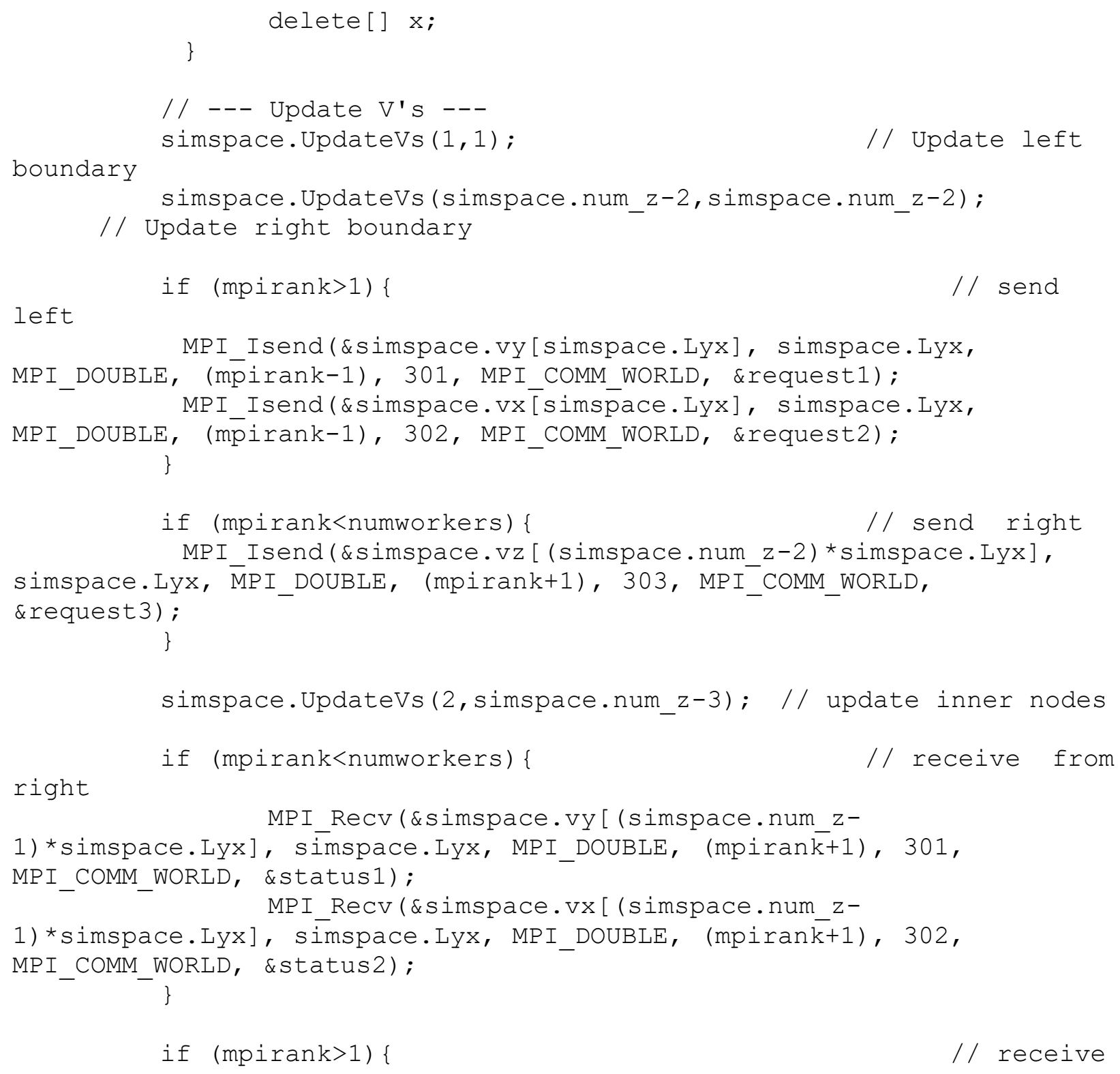




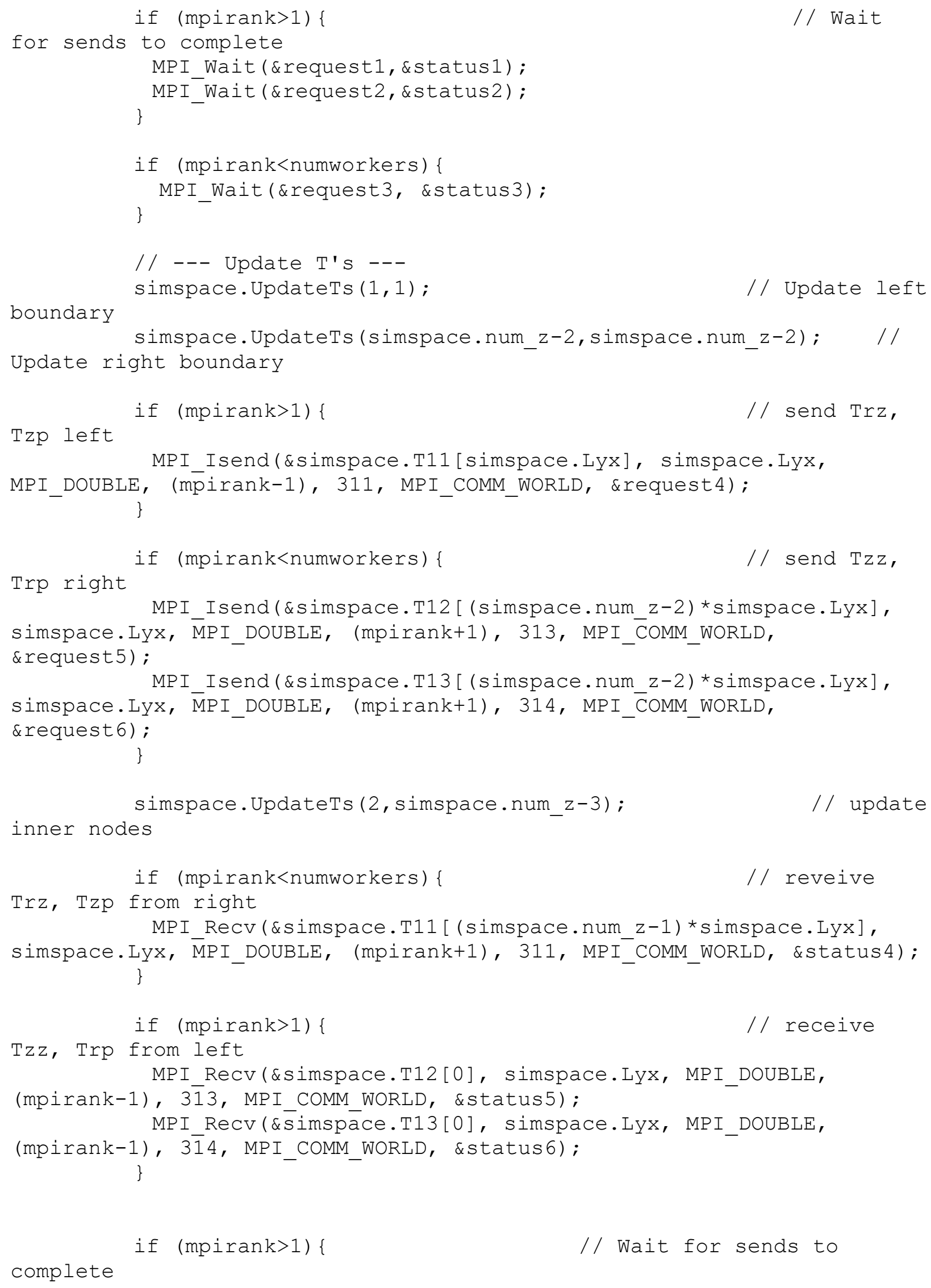




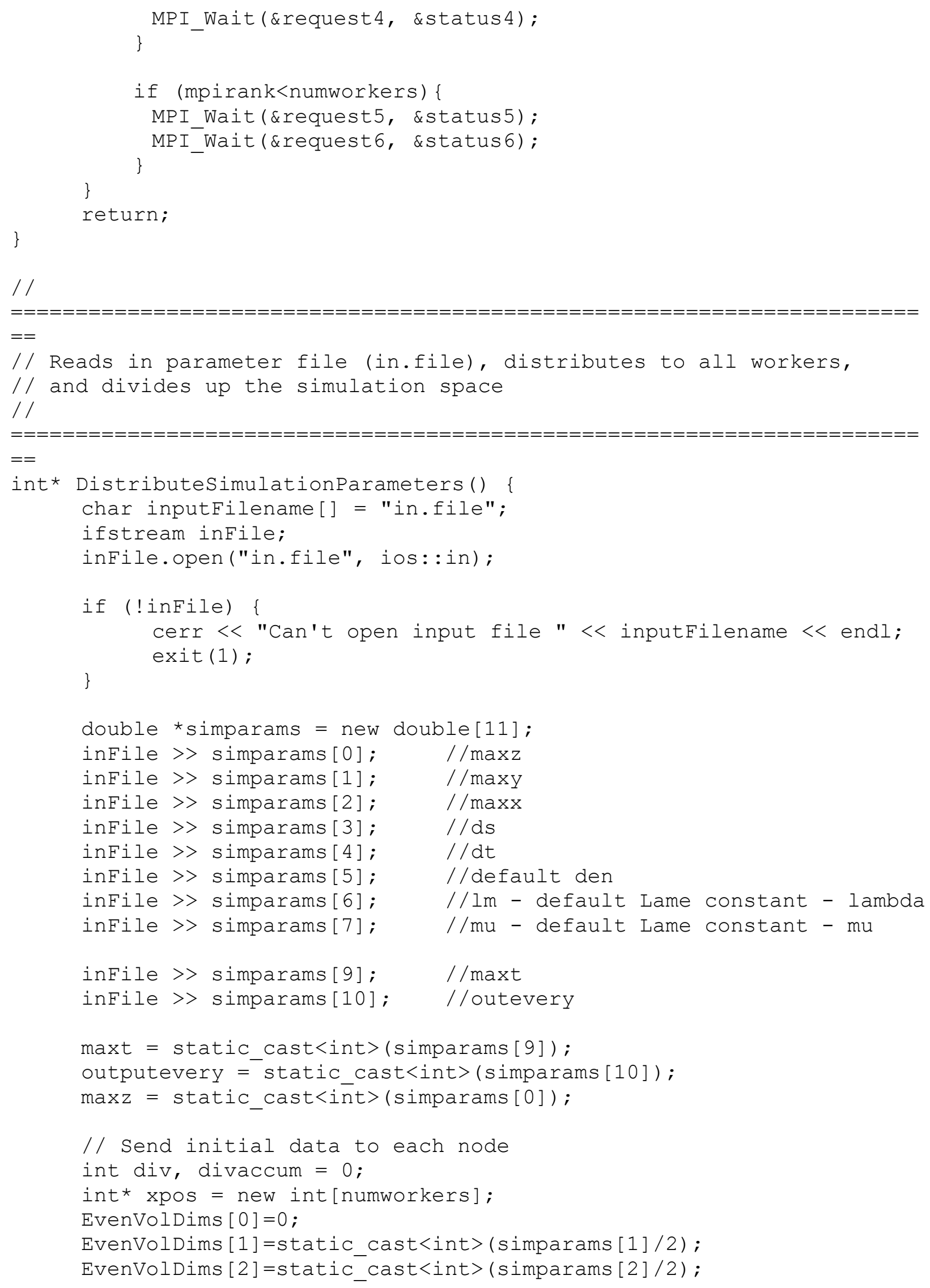




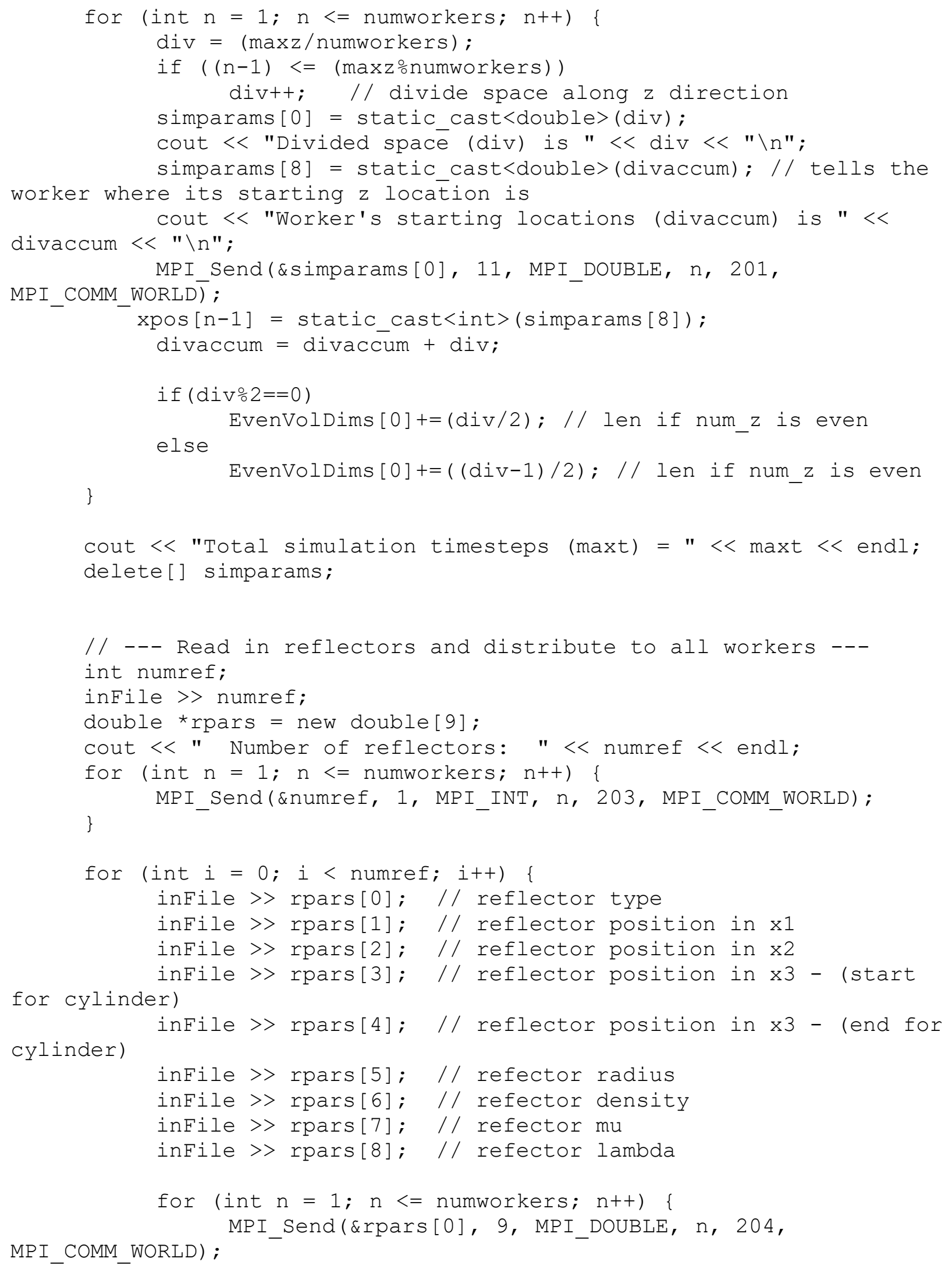




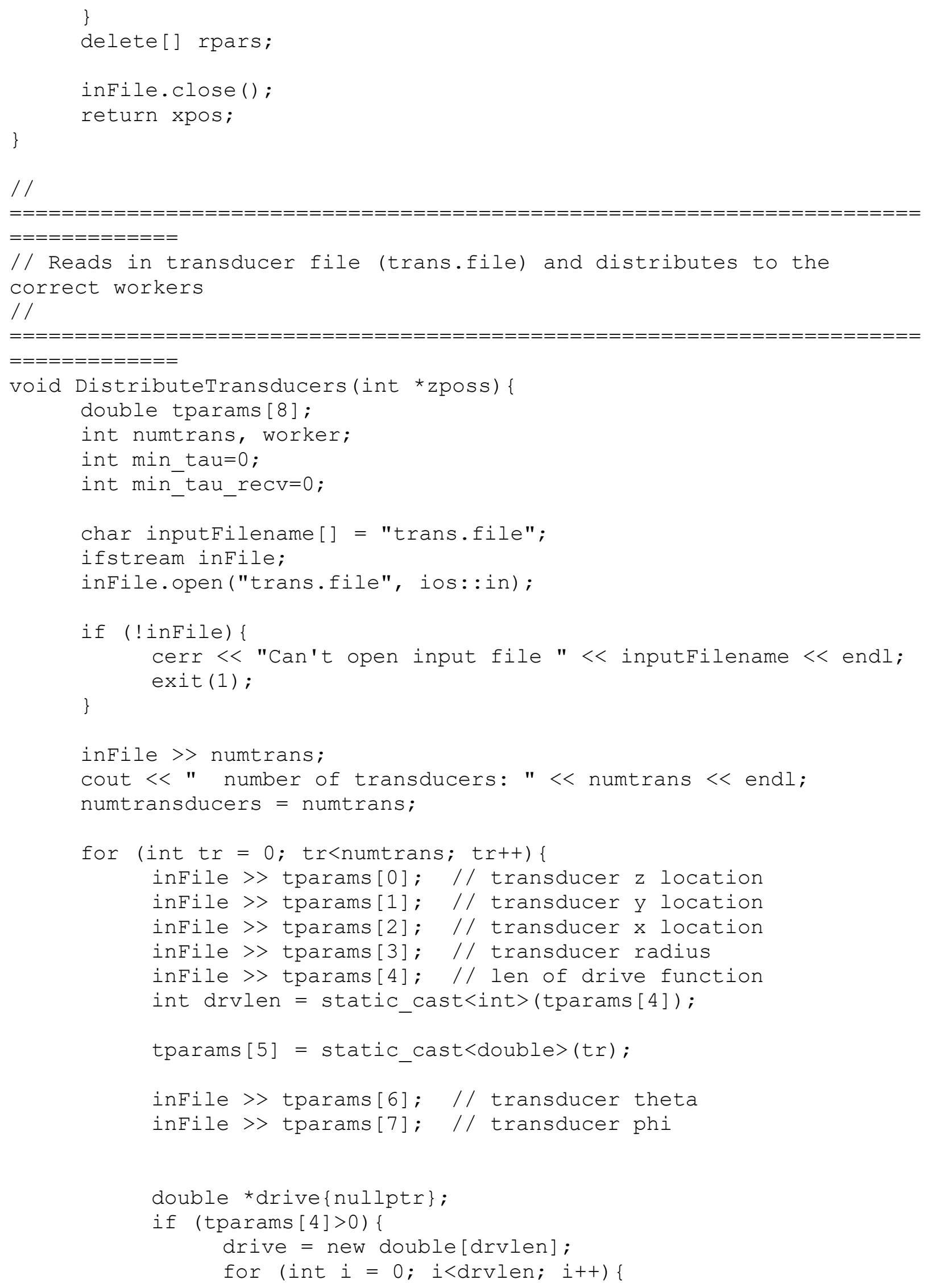




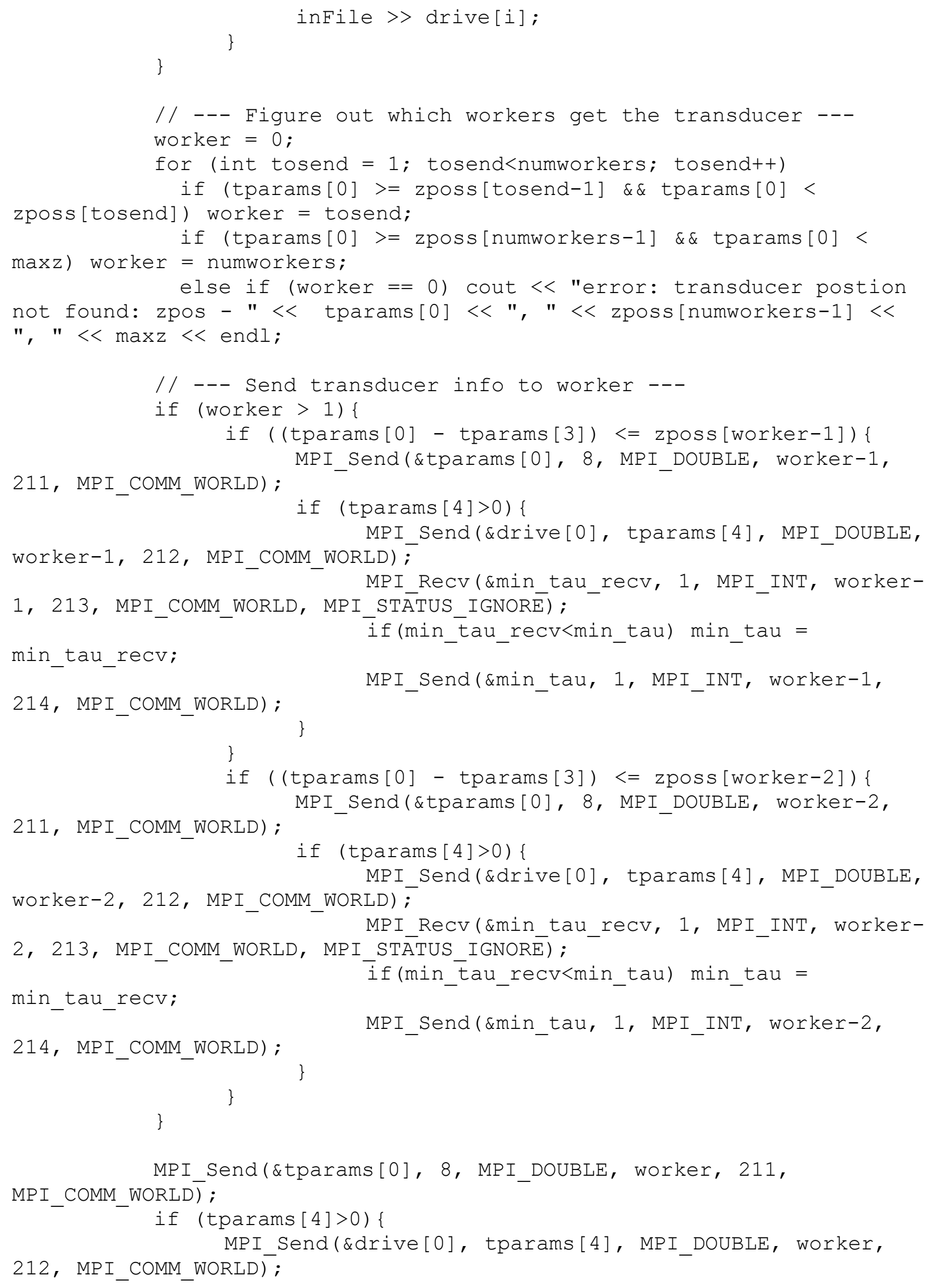




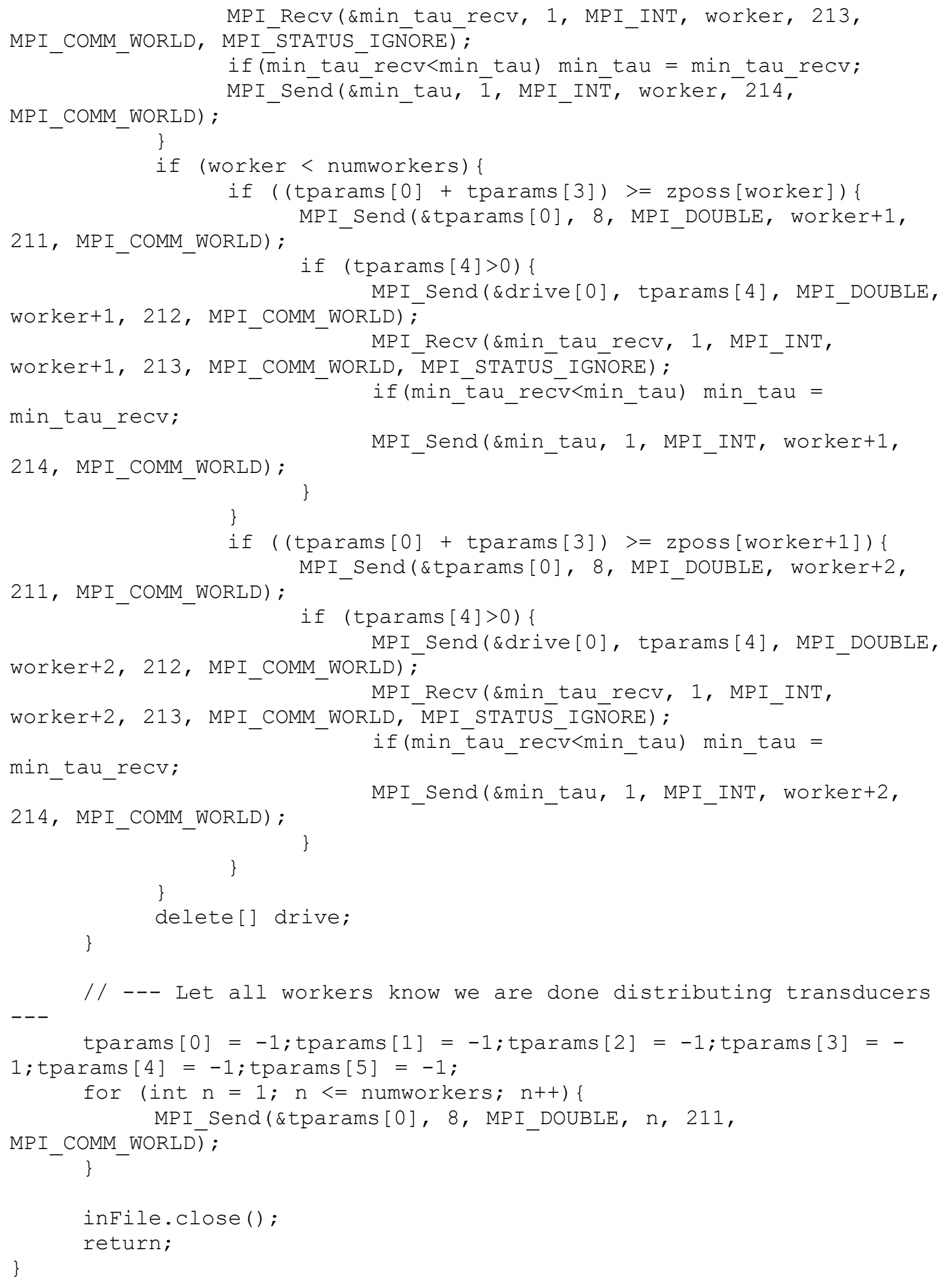




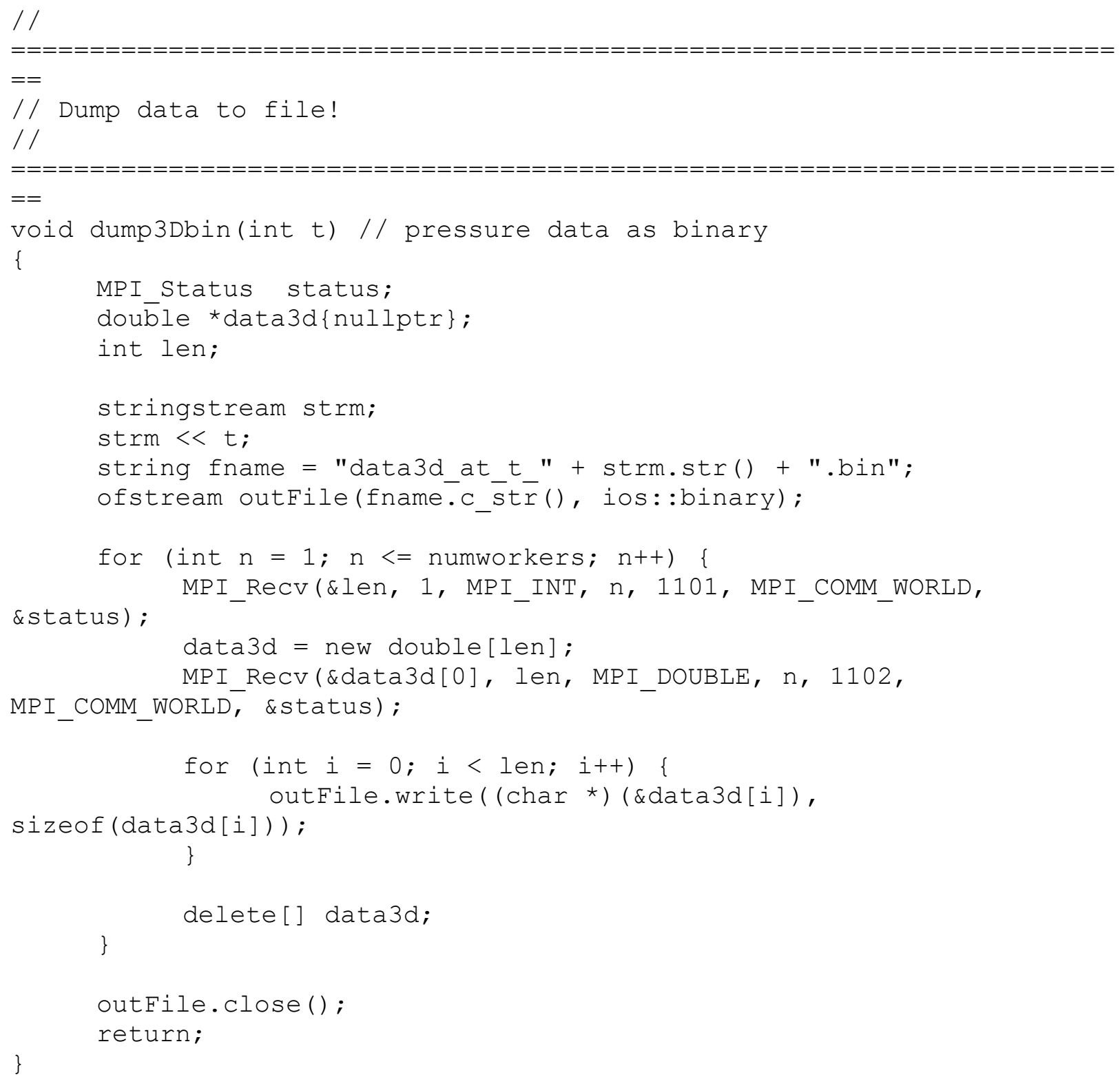




\section{C++ Code: 'space.h'}

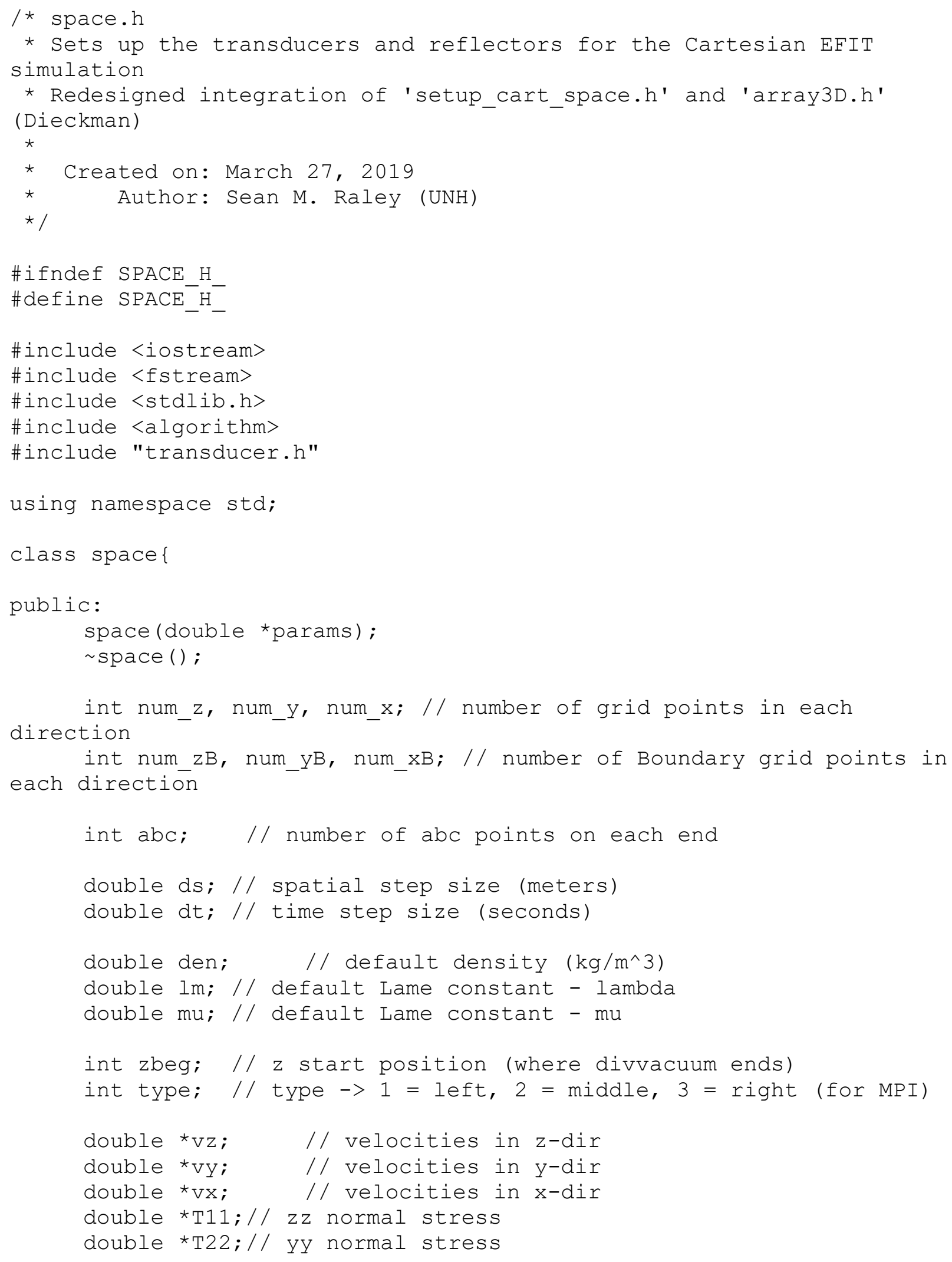




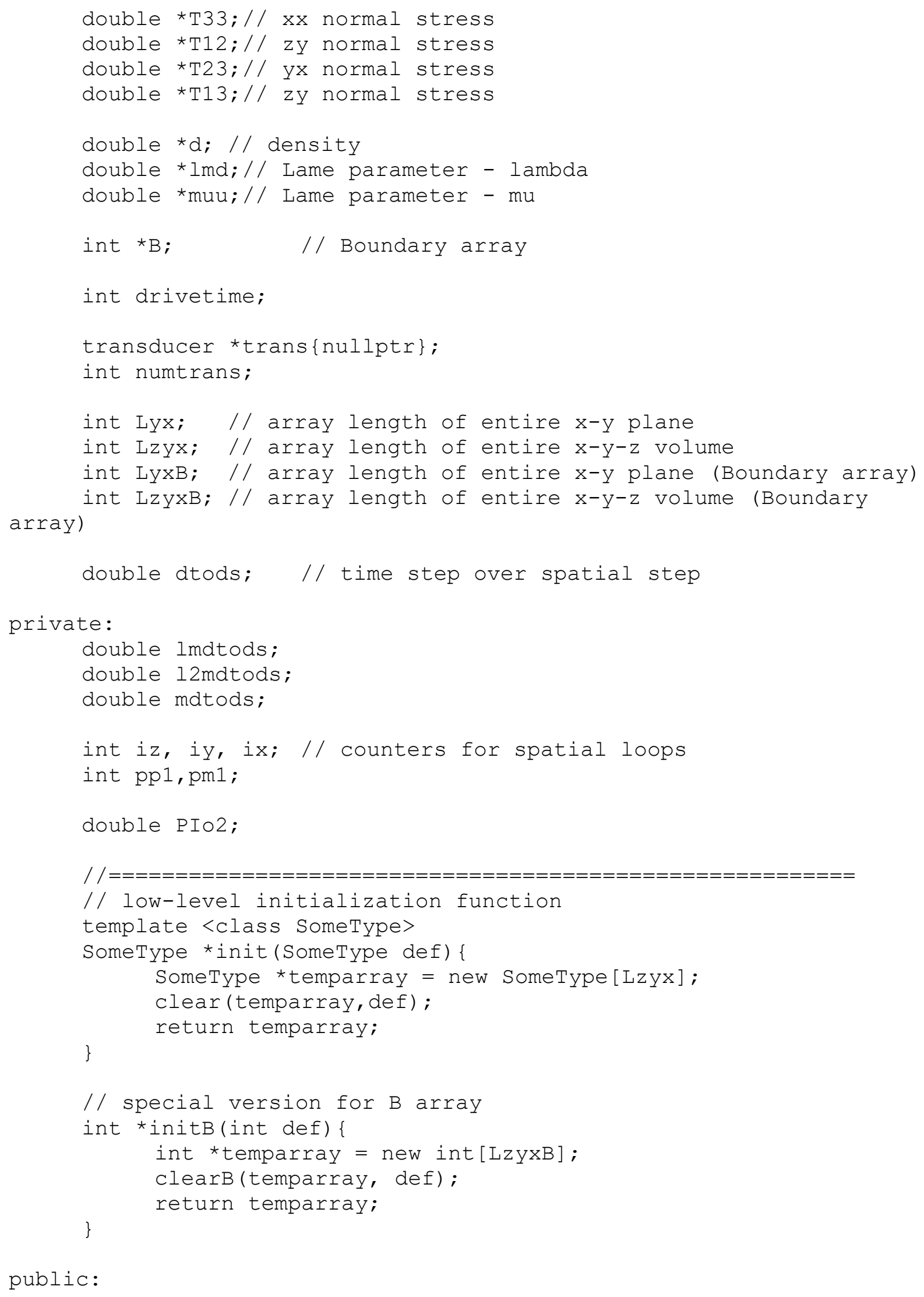

public: 


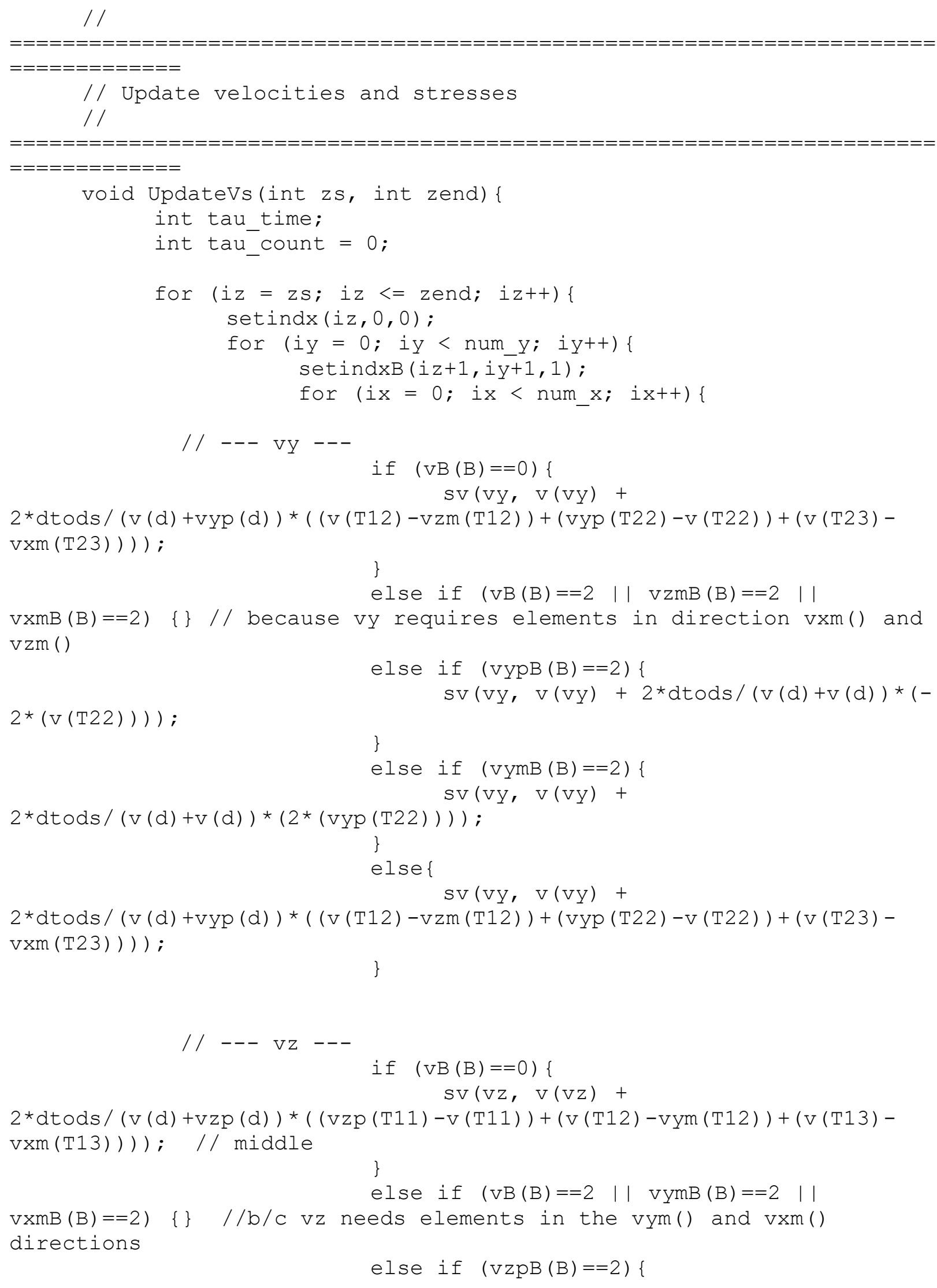




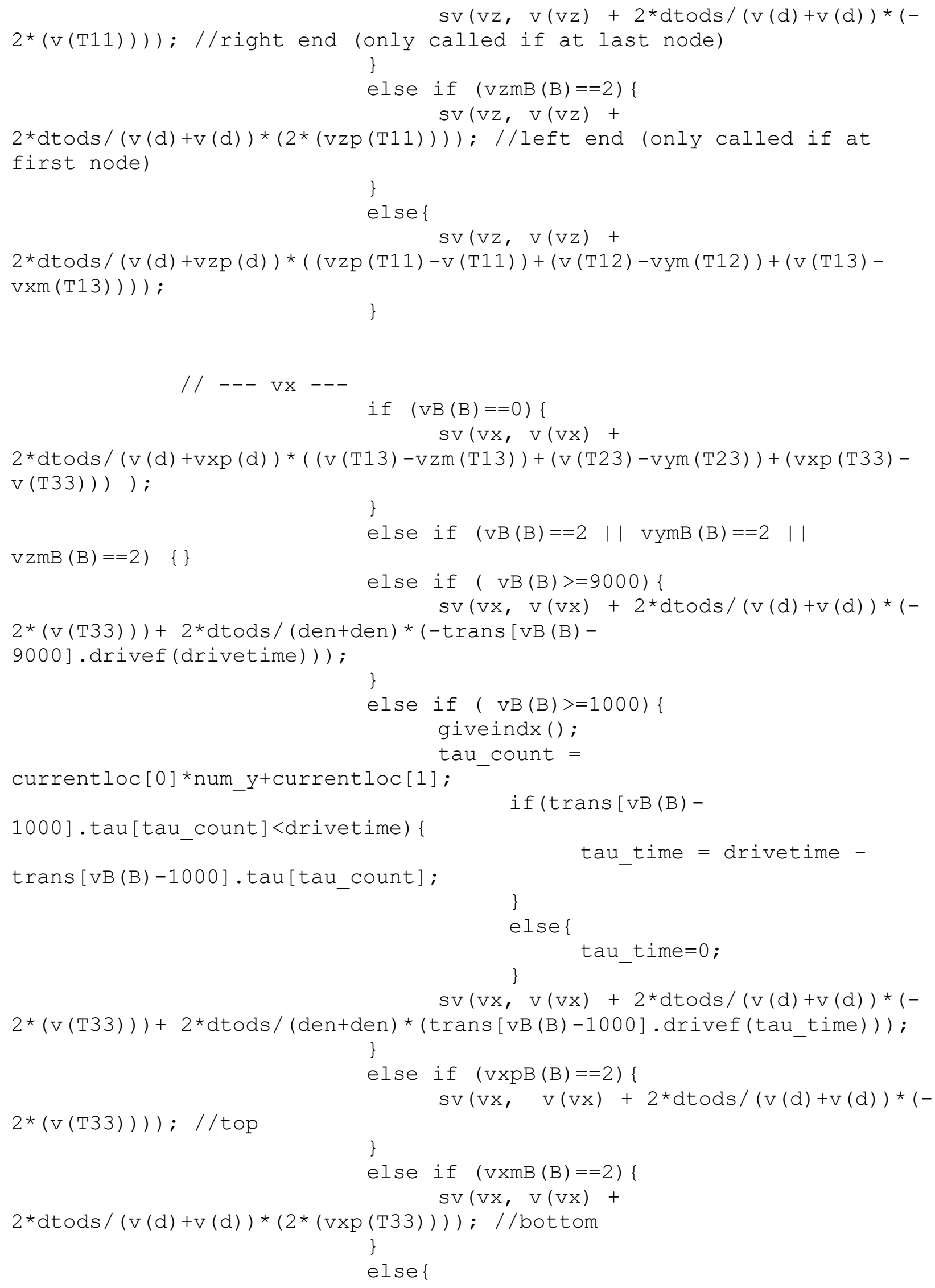




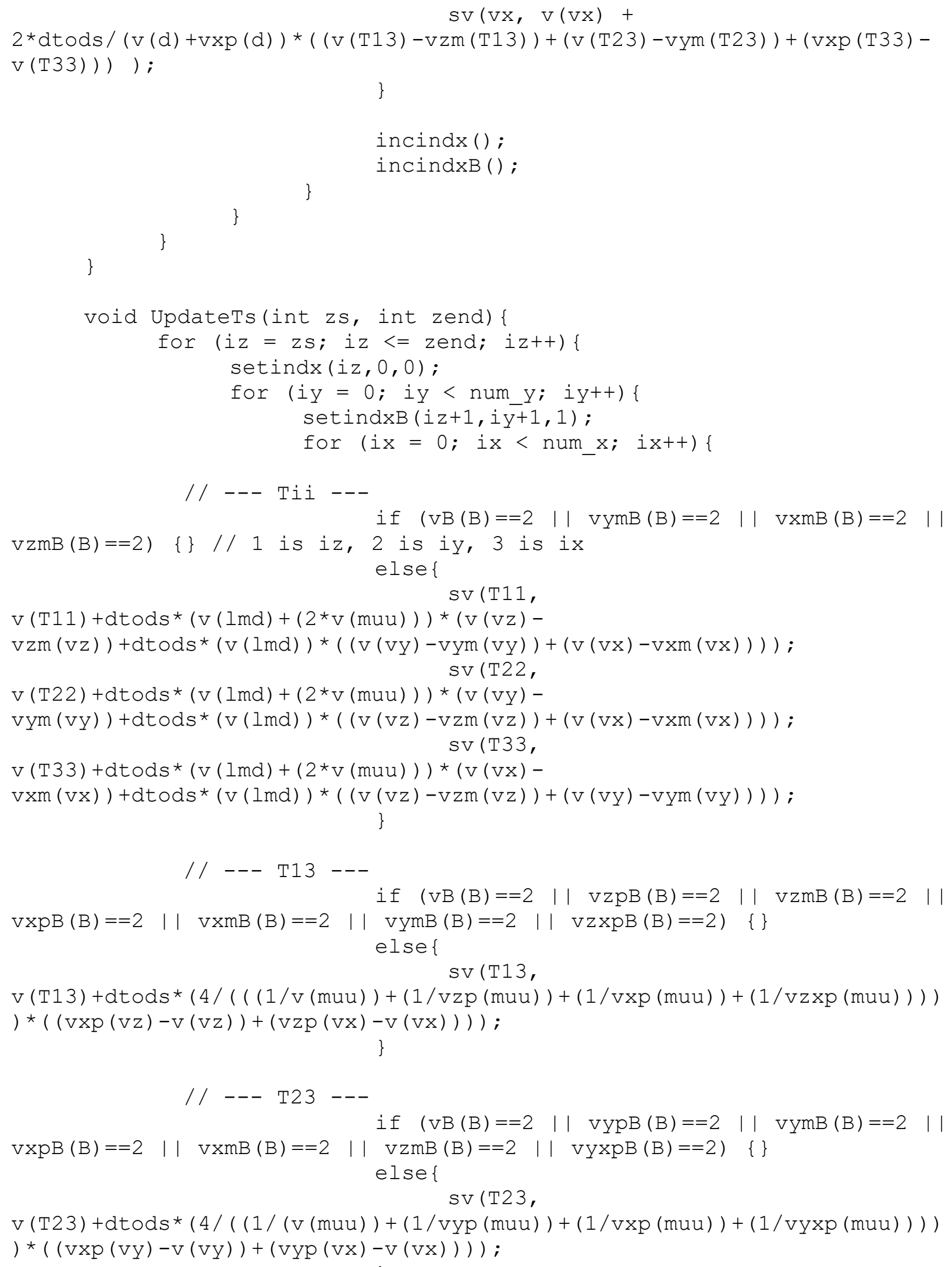




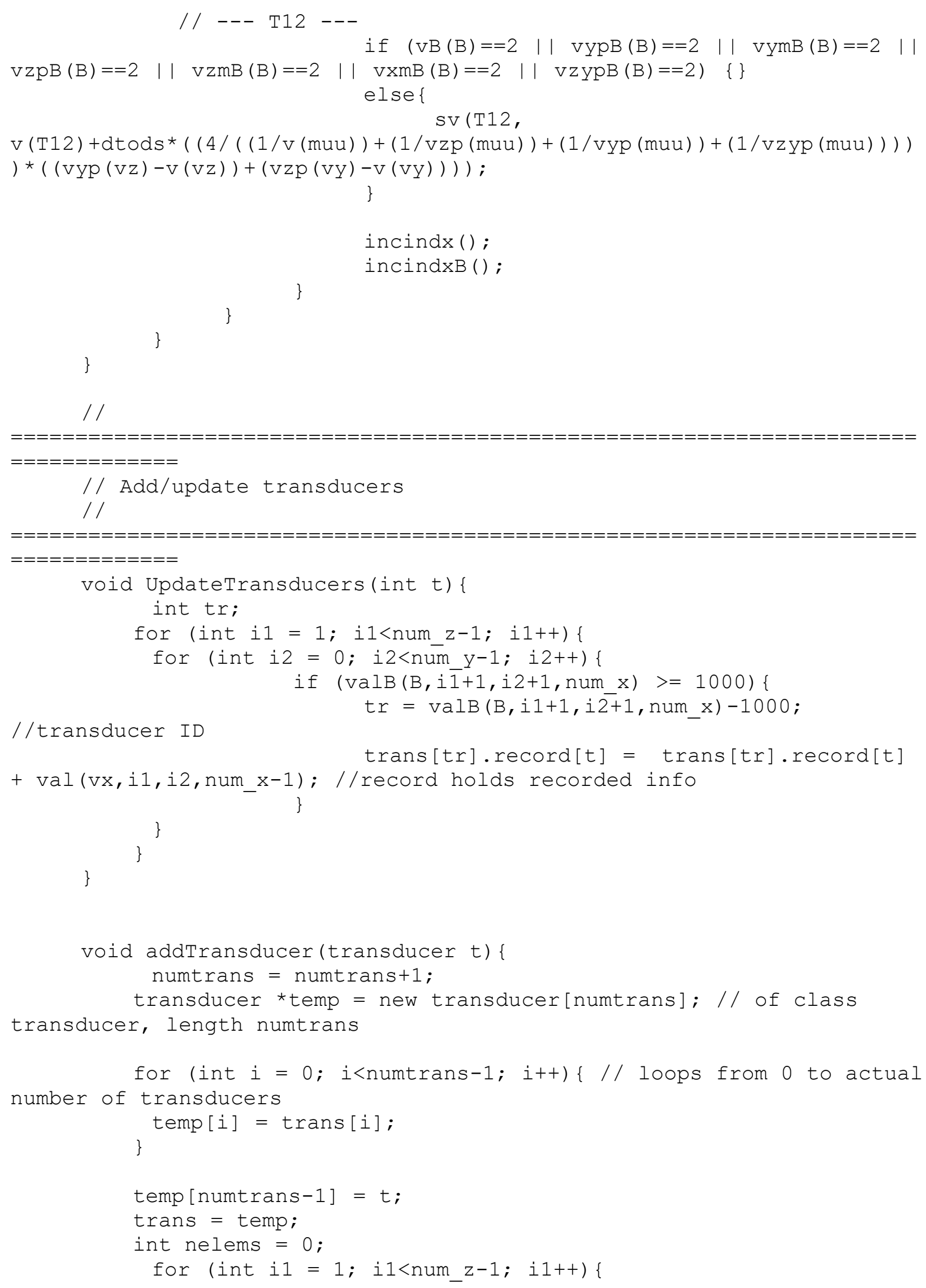


for (int i2 $=0$; i2<num_y; i2++) \{

if ( ( $i 1+z b e g-t r a n s[n u m t r a n s-1] \cdot p o s i 1) *(i 1+z b e g-$

trans [numtrans-1] • posi1) +(i2-trans [numtrans-1] • posi2)*(i2-

trans [numtrans-1].posi2)) <= (trans[numtrans-1].radius*trans[numtrans1].radius) ) \{

$\operatorname{set} B(B, i 1+1, i 2+1, t \cdot p o s i 3+1,1000+$ numtrans -1$)$;

//executes if $(z-z o)^{\wedge} 2+(y-y o)^{\wedge} 2<=\operatorname{rad}^{\wedge} 2$, ie, if within a circle of

transducer radius then set $B>1000$

\}

nelems++;

\}

if (nelems != trans [numtrans-1].tau_values) \{ cout<<"WARNING! For worker starting at

$z="<<$ zbeg $<<$ "Number of transducer elements defined by addTransducer() does NOT equal number of transducer elements in delay array tau.

Transducer errors likely."<<endl;

\}

temp [numtrans-1]. numelems = nelems;

\}

//

$=\mathrm{C}=\mathrm{=}=\mathrm{=}=\mathrm{=}=\mathrm{=}=\mathrm{=}=\mathrm{=}=\mathrm{=}=\mathrm{=}=\mathrm{=}=\mathrm{=}=\mathrm{=}=\mathrm{=}=\mathrm{=}=\mathrm{=}=\mathrm{=}=\mathrm{=}=\mathrm{=}=\mathrm{=}=\mathrm{=}=\mathrm{=}=\mathrm{=}=\mathrm{=}=\mathrm{=}=\mathrm{=}=\mathrm{=}$

$=========$

// Add/update relectors - different types defined below

//

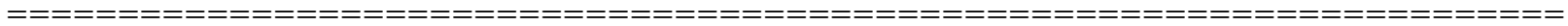

$========$

void addReflector(double typ, double p1, double p2, int start3, int end3, double rad, double dd, double mu, double lambda) \{
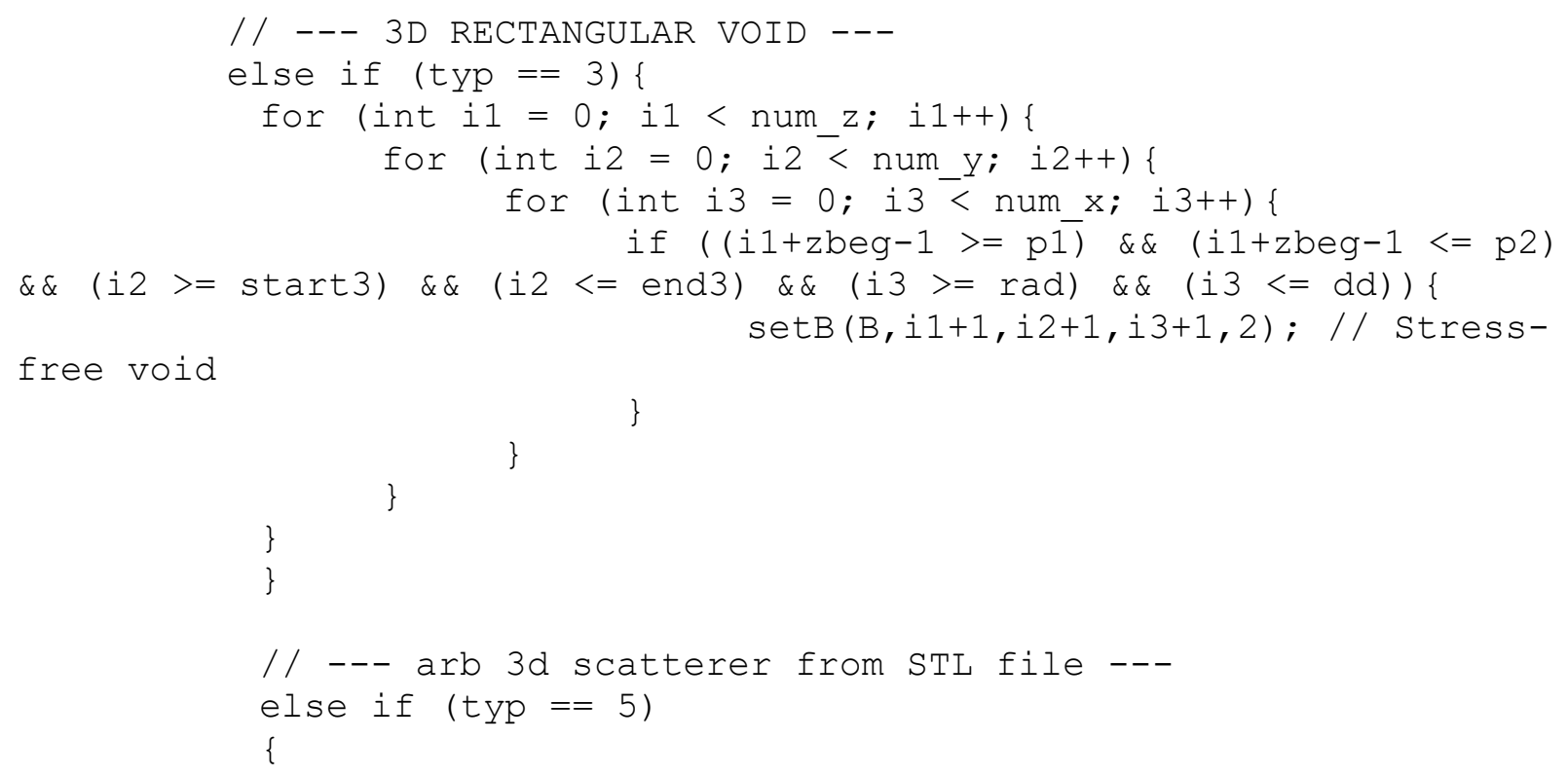


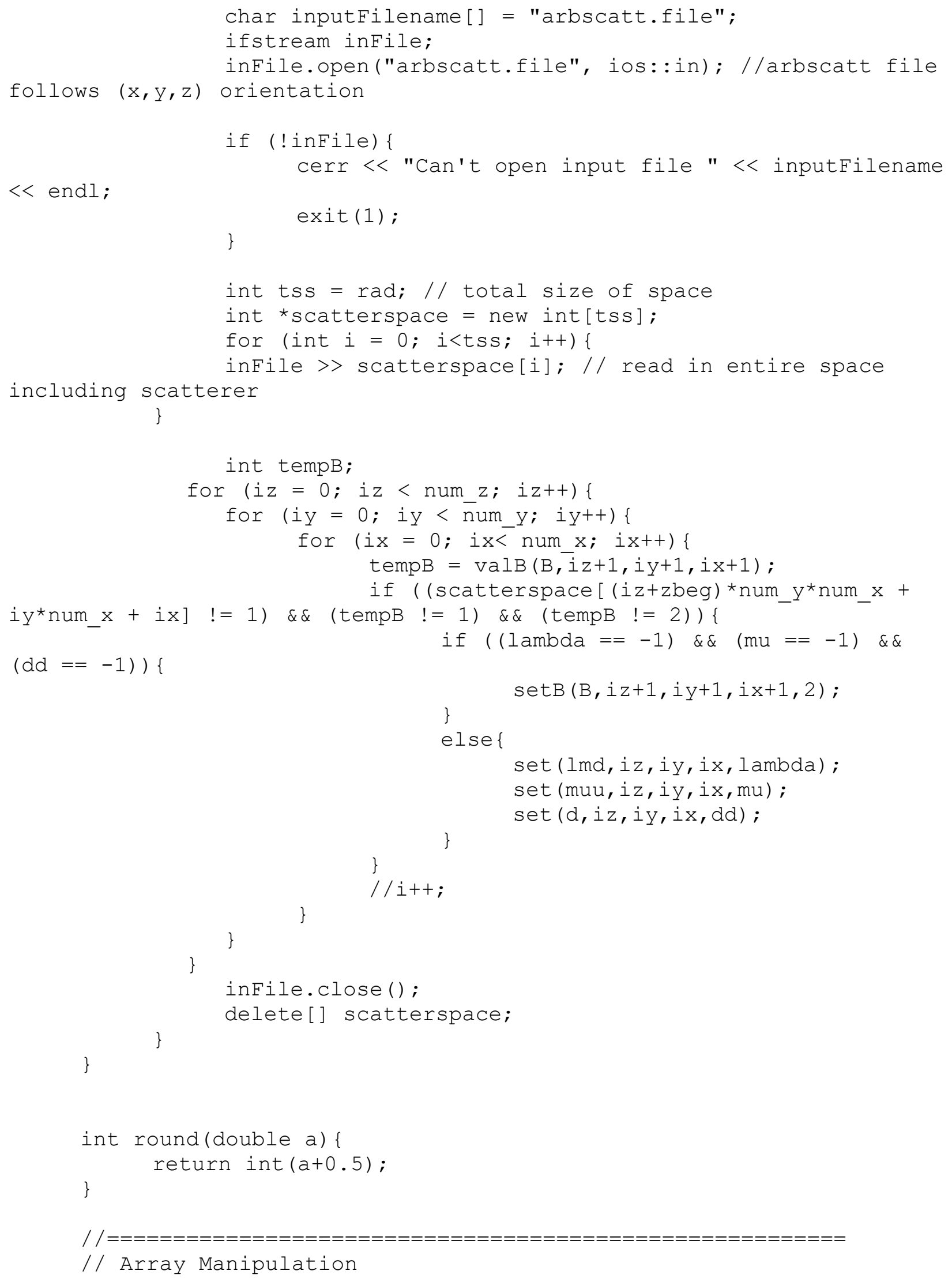




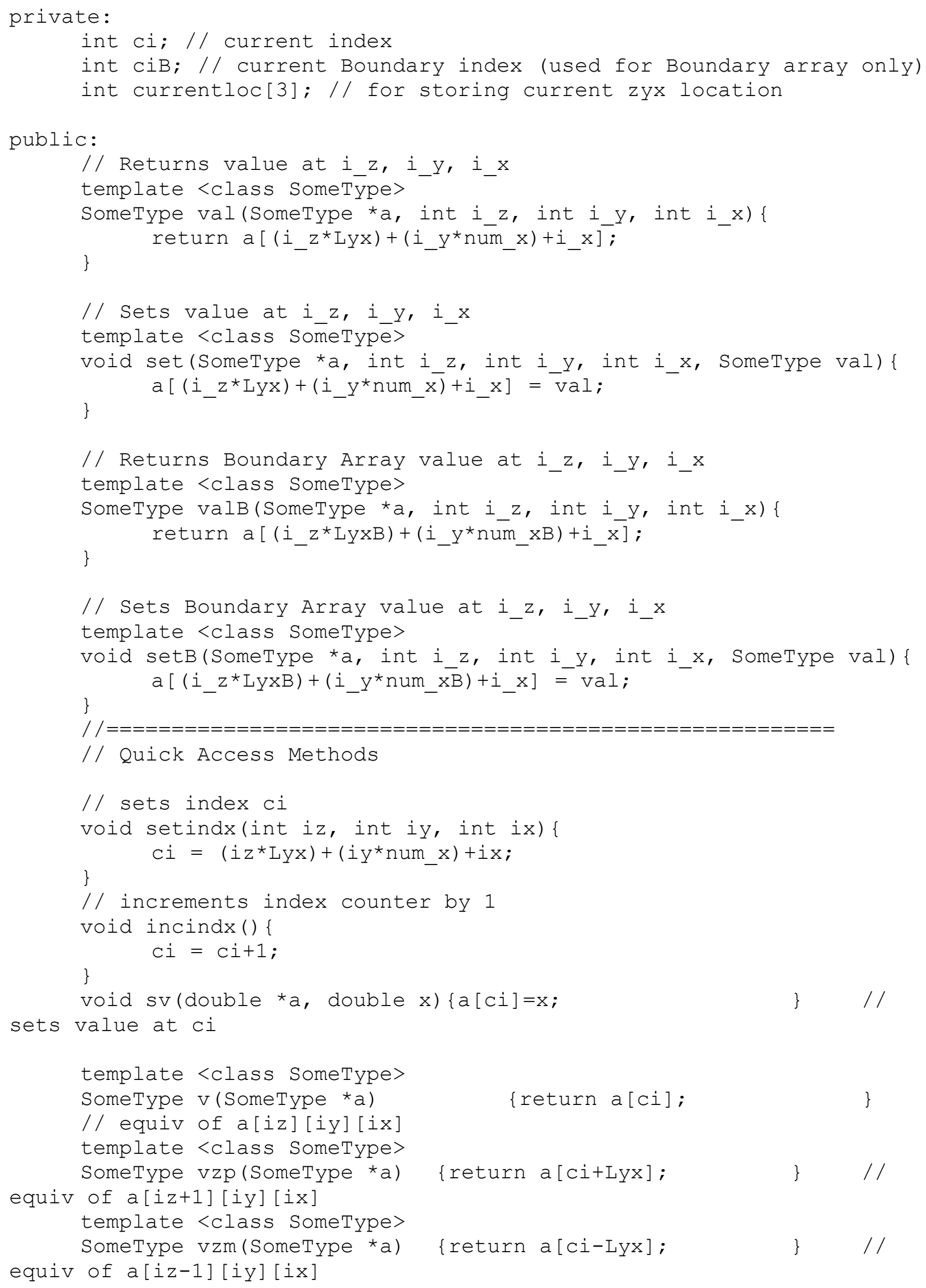




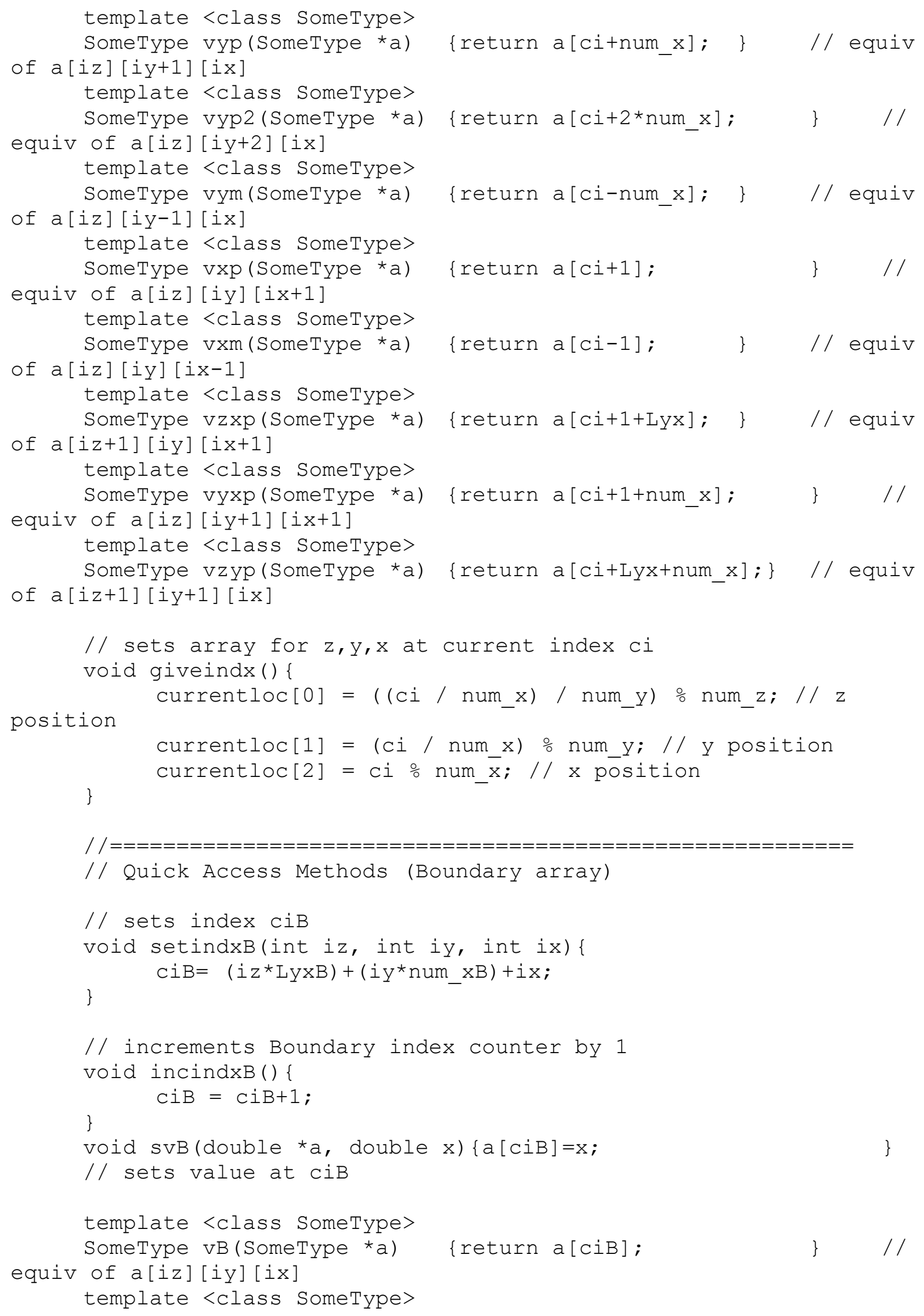


SomeType vzpB(SomeType *a) \{return a [ciB+LyxB]; //

equiv of a[iz+1][iy][ix]

template <class SomeType>

SomeType vzmB (SomeType *a) \{return a[ciB-LyxB]; //

equiv of a[iz-1][iy][ix]

template <class SomeType>

SomeType vypB (SomeType *a) \{return a[ciB+num_xB]; / /

equiv of a[iz][iy+1][ix]

template <class SomeType>

SomeType vyp2B (SomeType *a) \{return a $\left[\mathrm{ciB}+2^{\star}\right.$ num_xB]; \} //

equiv of a[iz][iy+2][ix]

template <class SomeType>

SomeType vymB (SomeType *a) \{return a[ciB-num_xB]; //

equiv of a[iz][iy-1][ix]

template <class SomeType>

SomeType vxpB (SomeType *a) \{return a[ciB+1]; //

equiv of a[iz][iy][ix+1]

template <class SomeType>

SomeType vxmB (SomeType *a) \{return a[ciB-1]; //

equiv of a[iz][iy][ix-1]

template <class SomeType>

SomeType vzxpB (SomeType *a) \{return a [ciB+1+LyxB]; /

equiv of a[iz+1][iy][ix+1]

template <class SomeType>

SomeType vyxpB (SomeType *a) \{return a $[\mathrm{ciB}+1+$ num_xB]; \} //

equiv of a[iz] [iy+1][ix+1]

template <class SomeType>

SomeType vzypB (SomeType *a) \{return a [ciB+LyxB+num_xB];

equiv of a[iz+1][iy+1][ix]

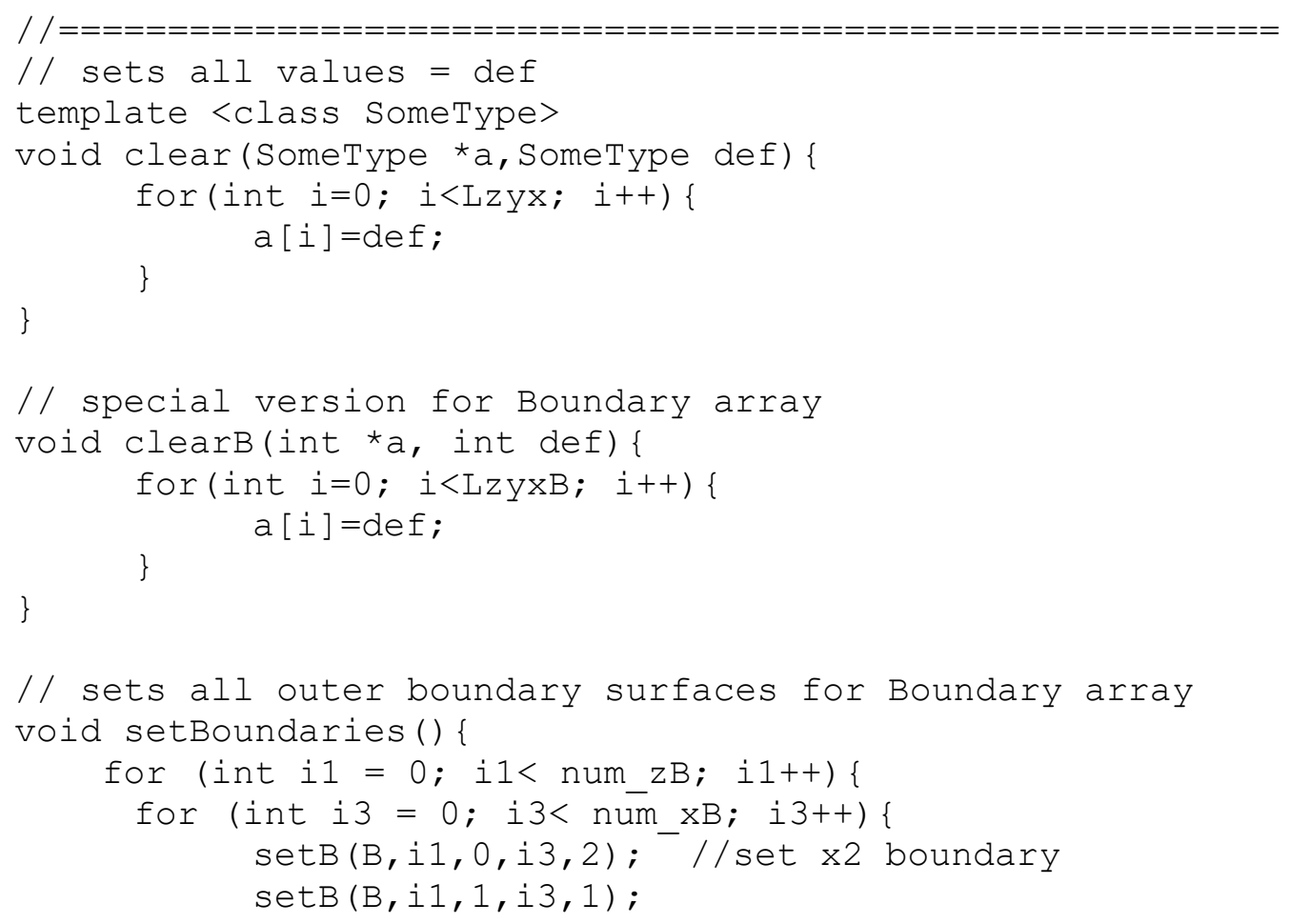




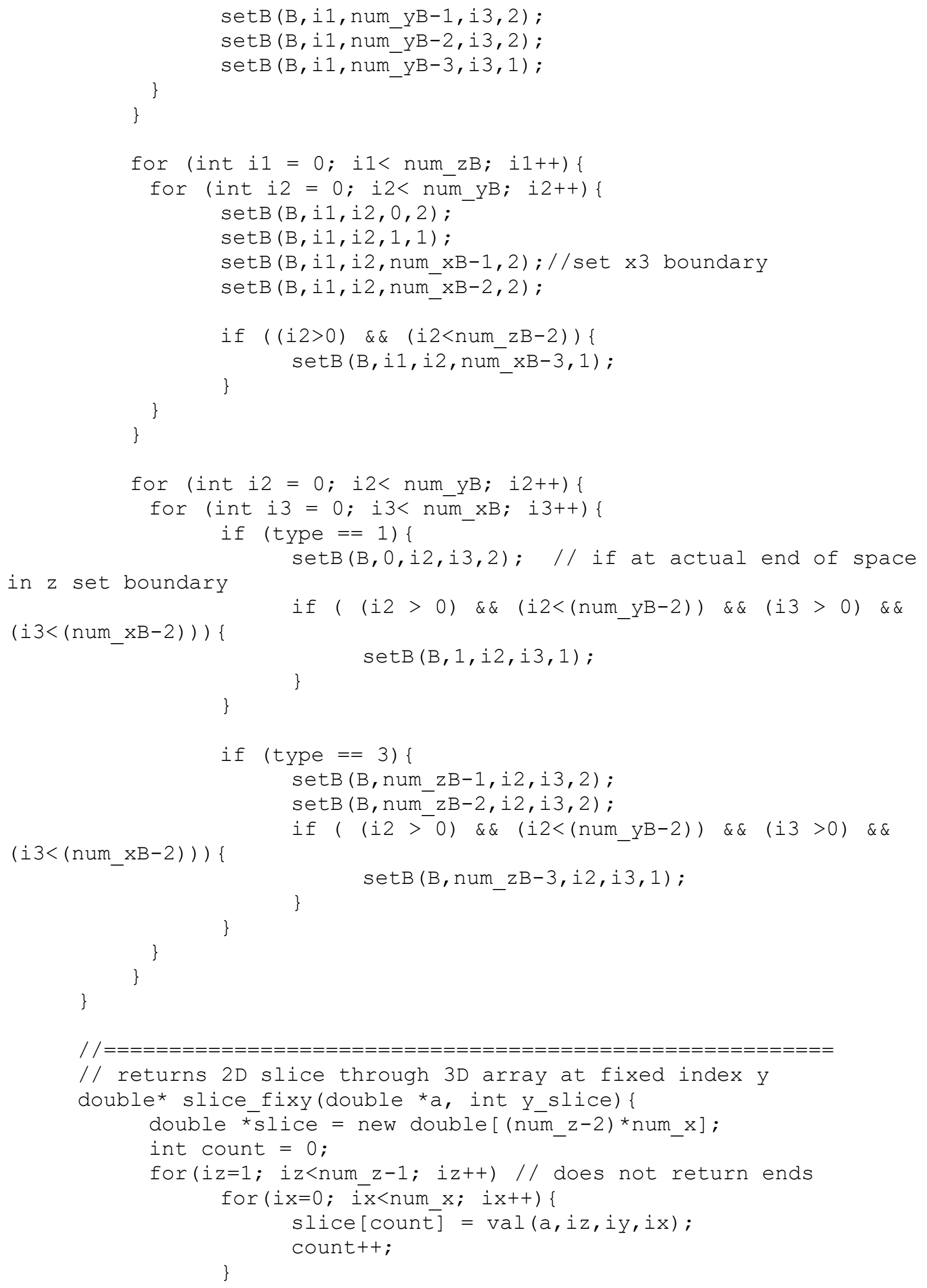




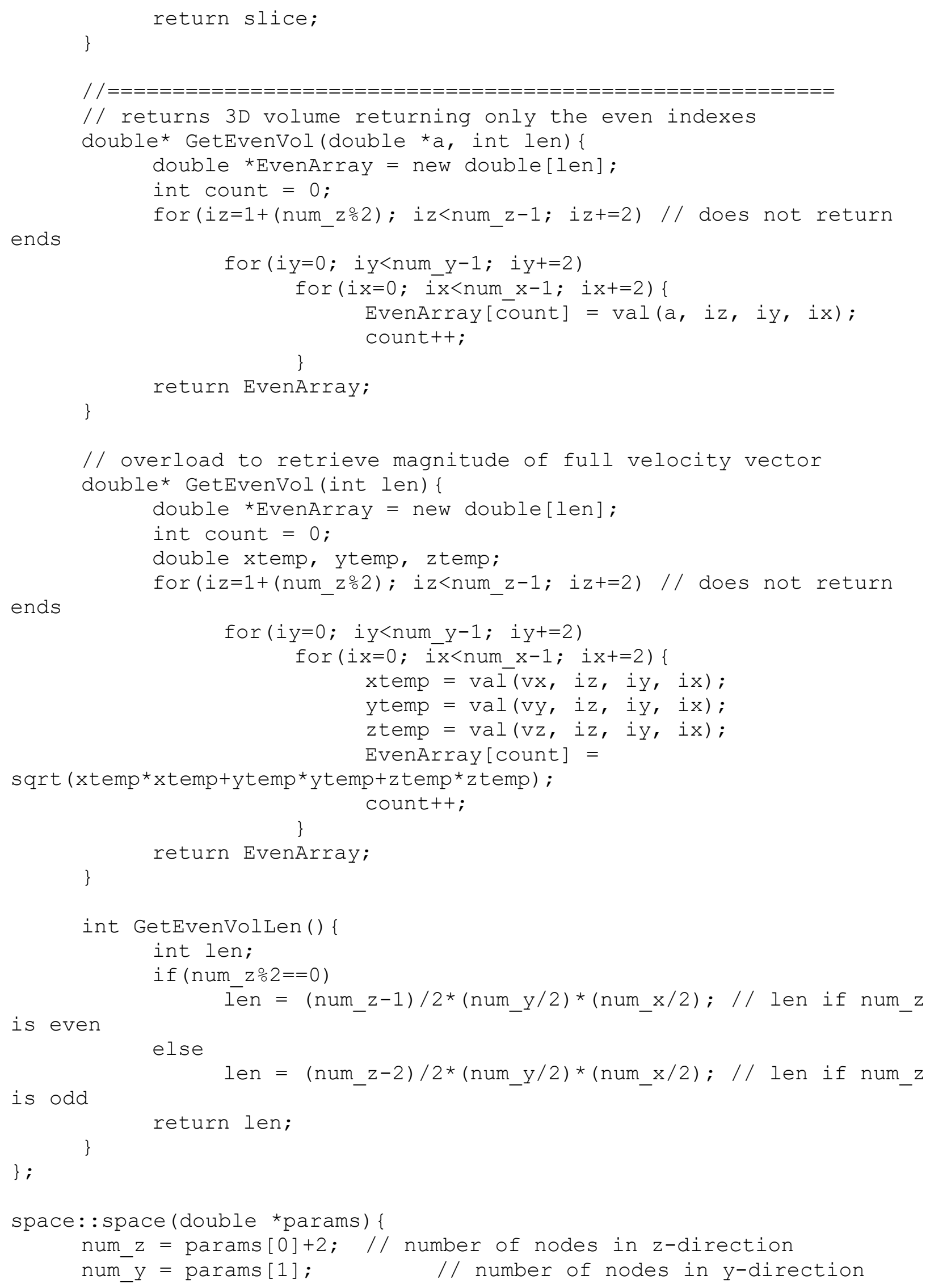




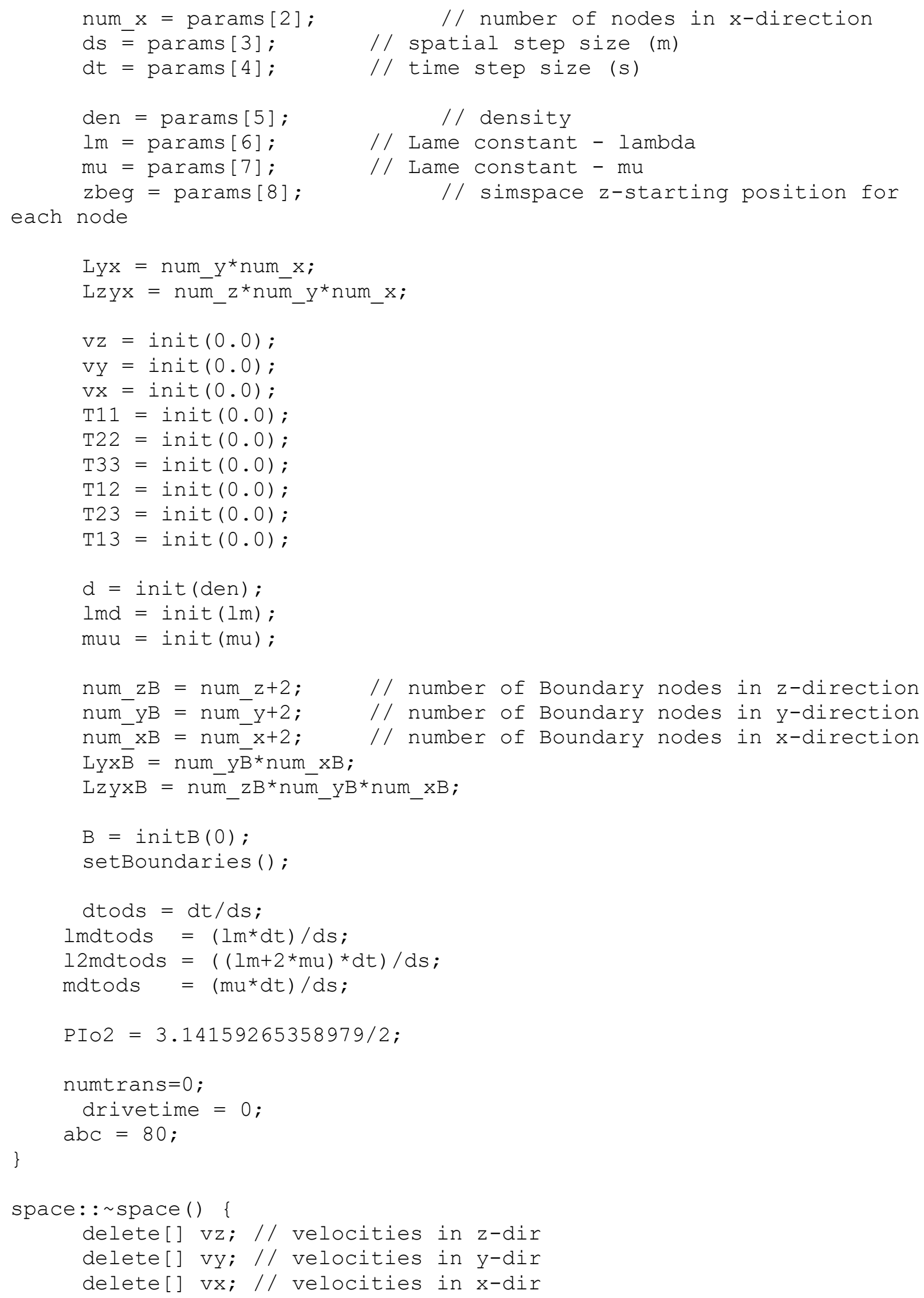




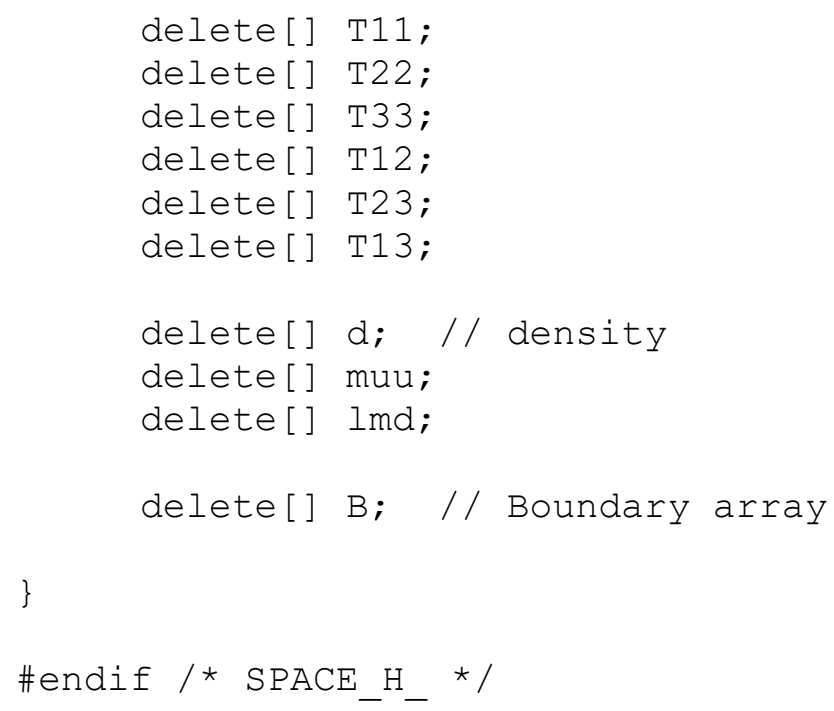




\section{C++ Code: 'transducer.h'}

/* 'transducer.h'

Custom transducer class for the Cartesian EFIT simulation

Cleaned up and heavily modified version of 'transducer.h' (Bertoncini, Campbell-Leckey, Miller, etc)

Eric A. Dieckman (WM)

Last edited: 10 Apr 2019 SMR

*

using namespace std;

class transducer \{

public:

double *drive\{nullptr\}; // array that holds drive function

int $\mathrm{dflen}=0$;

// length of drivefunc

double posil=0; // transducer center (z-direction)

double posi2=0; // transducer center (y-direction)

double posi3=0; // transducer center (x-direction)

double radius $=0 ; \quad$ // transducer radius - meters

bool driven=false; // driven: true=active (pitch or

pitch/catch), false=passive (catch)

int transID $=0$;

int numelems=0; // number of elements in simulation

space

double *record\{nullptr\};// array that holds recorded value

double theta=0; // beam angle from normal / x-direction radians

double phi=0; // beam angle from (+) z-direction -

radians

int num_y $=0 ; \quad / / y$ elements in this worker

int num_z $=0 ; \quad / / \mathrm{z}$ elements in this worker

int * taú $\{$ nullptr\}; // yz array of element time delays for

drive function

int zbeg $=0$; // starting location for this worker

in $z$-direction

int tau values=0; // number of elements which have

values in the tau array (equal to number of transducer elements on

this node)

double dtods=0;

transducer() \{ // blank constructor

\}

transducer(double $x 1$, double $x_{2}$, double $x_{3}$, double rad, int tID, int maxt, double angle1, double angle2, int dim_y, int dim_z, int z_start, double timebyspace) \{

$$
\begin{aligned}
& \text { posil }=x 1 ; \\
& \text { posi2 }=x 2 ;
\end{aligned}
$$




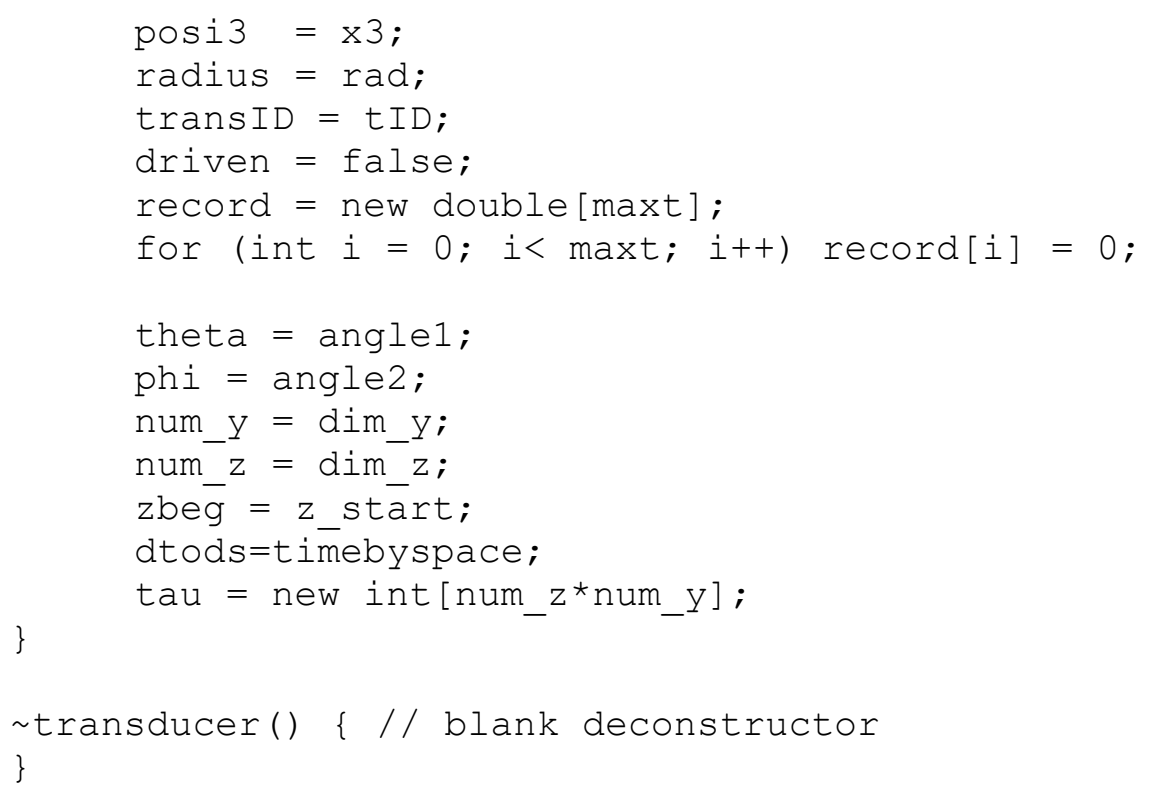


for (int $\mathrm{k}=0 ; \mathrm{k}<\mathrm{num} z \mathrm{z} ; \mathrm{k}++$ ) \{

for (int $j=0 ; j<$ num_y; $j++)\{$

if $(((k+z b e g-$ posil $) *(k+z b e g-p o s i 1)+(j-$ posi2 $) *(j-$ posi2) $)<=($ radius*radius $))\{$

temp_tau=static_cast<int $>($ floor $(((k+z b e g-$ posil $) * \cos ($ phi $)+(j-$ posi2)*sin $($ phi $)) * \sin ($ theta $) /$ cL/dtods $)) ;$

tau $\left[k^{\star}\right.$ num_y+j] $=$ temp_tau;

if (temp_tau<min_tau_local) \{

\} min_tau_local=temp_tau;

tau_values++;

\}

else

$\operatorname{tau}\left[k^{*}\right.$ num_y+j] $=0 ; / /$ if not within the

circle of the transducer

\}

\}

\}

\}

return min_tau_local;

\} ; 


\section{SELECTED REFERENCES}

[1] C. J. Hellier, Handbook of Nondestructive Evaluation, 2nd ed. McGraw-Hill Professional Publishing, 2012.

[2] S. r. o. STARMANS electronics, "Railway Rail Testing," 2019. [Online]. Available: http://www.starmans.net/applications/railway-rail-testing/. [Accessed: 31-May-2019].

[3] Nordco Rail Flaw Defects Identification Handbook. Nordco Rail Services.

[4] P. M. Morse, K. U. Ingard, and F. B. Stumpf, "Theoretical Acoustics," Am. J. Phys., vol. 38, no. 5, pp. 666-667, 1970.

[5] C. J. Partridge, "Sound Wave Scattering from a Rigid Sphere,” Maribyrnong, Vic., 1993.

[6] P. A. Martin, "Acoustic scattering by a sphere in the time domain," Wave Motion, vol. 67, pp. 68-80, 2016.

[7] P. Fellinger, R. Marklein, K. J. Langenberg, and S. Klaholz, "Numerical modeling of elastic wave propagation and scattering with EFIT - elastodynamic finite integration technique," Wave Motion, vol. 21, no. 1, pp. 47-66, 1995.

[8] P. Thoma and T. Weiland, "A subgridding method in combination with the finite integration technique," 1995 25th Eur. Microw. Conf., vol. 2, no. 1, pp. 770-774, 1995.

[9] K. S. Yee, "Numerical Solution of Initial Boundary Value Problems Involving Maxwell's Equations in Isotropic Media,” IEEE Transactions on Antennas and Propagation. 1966.

[10] W. Yu, Advanced FDTD Methods: Parallelization, Acceleration, and Engineering Applications. Artech House, 2011.

[11] T. Weiland, M. Timm, and I. Munteanu, “A practical guide to 3-D simulation," IEEE Microw. Mag., vol. 9, no. 6, pp. 62-75, Nov. 2008.

[12] R. Marklein, “The Finite Integration Technique as a General Tool to Compute Acoustic, 
Electromagnetic, Elastodynamic, and Coupled Wave Fields," Rev. Radio Sci. 1999-2002 URSI, pp. 201-244, 2002.

[13] J.-F. Lee, R. Lee, and A. Cangellaris, "Time-Domain Finite-Element Methods," IEEE Trans. Antennas Propag., vol. 45, no. 3, pp. 430-442, 1997.

[14] L. E. Kinsler, A. R. Frey, A. B. Coppens, and J. V. Sanders, Fundamentals of acoustics 4th edition. 2000.

[15] K. E. Rudd, "Parallel three-dimensional acoustic and elastic wave simulation methods with applications in nondestructive evaluation," 2003.

[16] H. Childs et al., "VisIt: An End-User Tool For Visualizing and Analyzing Very Large Data," in High Performance Visualization--Enabling Extreme-Scale Scientific Insight, 2012, pp. 357-372.

[17] E. Gabriel et al., "Open MPI: Goals, concept, and design of a next generation MPI implementation," Recent Adv. Parallel Virtual Mach. Messag. Passing Interface, pp. 97104, 2004.

[18] J.-P. Berenger, "A Perfectly Matched Layer for the Absorption of Electromagnetic Waves," J. Comput. Phys., vol. 114, no. 2, pp. 185-200, 1994.

[19] University of Texas at El Paso, "Finite-Difference Time-Domain Poster." [Online]. Available: http://emlab.utep.edu/academics.htm. [Accessed: 01-Jun-2019].

[20] Sanpaz, "Components of the Cauchy stress tensor in Cartesian coordinates," Wikipedia, 2009. [Online]. Available: https://commons.wikimedia.org/wiki/File:Components_stress_tensor_cartesian.svg. [Accessed: 01-Jun-2019].

[21] S. Garrett, Understanding Acoustics: An Experimentalist's View of Acoustics and 
Vibration (Graduate Texts in Physics), 1st ed. Springer, 2017.

[22] A. Briggs and O. Kolosov, Acoustic Microscopy: Second Edition. 2010.

[23] F. Jones, "Ray Paths in Layered Media: Reflections and refractions at a plane interface," UBC Earth and Ocean Sciences, 2018. [Online]. Available:

https://www.eoas.ubc.ca/courses/eosc350/content/methods/meth_6/raypaths.html. 